\title{
동 2014
}

TECHNISCHE

UNIVERSITATT

DARMSTADT

$11^{\text {th }}$ International Conference on

Computability and Complexity in Analysis

\author{
July $21-24,2014$
}

Proceedings 



\section{Preface}

Computability and Complexity in Analysis (CCA) investigates the fundamental capabilities and limitations of operations on continuous data. This provides a rigorous theory of computing for instance over real numbers, (say, continuous or smooth) functions, and (e.g., compact or open) subsets by approximation up to prescribable absolute error - inspired by, and with applications to, numerics. Initiated by Alan Turing, this field thus combines Theoretical Computer Science (recursion and complexity theory) with Analysis and its foundations by means of Mathematical Logic. Computability theory is the study of the limitations and abilities of computers in principle.

Computational complexity theory provides a framework for understanding the cost of solving computational problems, as measured by the requirement for resources such as time and space. The classical approach in these areas is to consider algorithms as operating on finite strings of symbols from a finite alphabet. Such strings may represent various discrete objects such as integers or algebraic expressions, but cannot represent general real or complex numbers, unless they are rounded. Most mathematical models in physics and engineering, however, are based on the real number concept. Thus, a computability theory and a complexity theory over the real numbers and over more general continuous data structures is needed. Despite remarkable progress in recent years many important fundamental problems have not yet been studied, and presumably numerous unexpected and surprising results are waiting to be detected. Scientists working in the area of computation on real-valued data come from different fields, such as theoretical computer science, domain theory, logic, constructive mathematics, computer arithmetic, numerical mathematics and all branches of analysis. The conference provides a unique opportunity for people from such diverse areas to meet, present work in progress and

exchange ideas and knowledge. The topics of interest include foundational work on various models and approaches for describing computability and complexity over the real numbers. They also include complexity-theoretic investigations, both foundational and with respect to concrete problems, and new implementations of exact real arithmetic, as well as further developments of already existing software packages. We hope to gain new insights into computability-theoretic aspects of various computational questions from physics and from other fields involving computations over the real numbers.

Starting in 1995 at the FernUniversität Hagen, annual meetings have continuously (pun!) taken place and quickly grown both in number and national variety of attendance into a truly international conference series on various locations throughout the world. The 11th International Conference on Computability and Complexity in Analysis was held on July 21-24, 2014 at TU Darmstadt (GERMANY); and the present proceedings contain the papers presented at CCA 2014: 6 invited speakers had kindly agreed to deliver talks and the program committee decided to accept 23 of the submitted works. A special session on Implementation of Exact Real Number Arithmetic, organized by Norbert Müller (Trier), demonstrated and fostered connections between theory and practice. We gratefully acknowledge financial support by both local departments of mathematics and computer science, by the International Research Training Group 1529, and by the German Research Foundation (DFG). 


\section{Conference schedule}

\section{July 21st (Monday): Topology \& Descriptive Set Theory}

08:30 Registration

09:00 Opening Address

09:30 Matthew de Brecht (Invited Talk):

Characterizations of the topological complexity

of discontinuous functions

10:30 Victor Selivanov:

On the Effective Hausdorff-Kuratowski Theorem

11:00 Coffee

11:30 Takayuki Kihara:

On the effectively $\mathrm{G}_{\delta}$-decomposable functions

12:00 Alexey Ostrovsky:

$L C_{n}$-measurable functions

12:30 Yasuyuki Tsukamoto and Hideki Tsuiki:

Strong properness of dyadic subbases

13:00 Lunch

14:30 Martin Pape:

Computability for Basic Quantum Mechanics

based on the Hilbert Lattice

15:00 Zvonko Iljazović and Bojan Pažek

Computable transition points

15:30 Matthias Schröder:

Characterising classes of hyperprojective qcb-spaces

16:00 Coffee

16:30 Arno Pauly (Invited Talk):

The Descriptive Theory of Represented Spaces

17:30 Debriefing and Open Problems
July 22nd (Tuesday) Morning Session:

Randomness, Probability, and Measure

09:00 Johanna Franklin (Invited Talk):

Techniques in randomness and ergodic theory

10:00 Coffee

10:30 Vasco Brattka, Guido Gherardi and Rupert Hölzl: Probabilistic Computability and Choice

11:00 Akitoshi Kawamura and Kentarô Yamamoto: On the Computational Power of Algorithmically Random Constants in Blum-Shub-Smale Machines

11:30 Nathanael Ackerman, Cameron Freer and Daniel Roy

On computability and disintegration

12:00 Lunch

\section{Afternoon Session: Logic in Analysis}

13:30 Martín Escardó (Invited Talk):

A constructive manifestation of the Kleene-Kreisel continuous functionals

14:30 Coffee

15:00 Stéphane Le Roux and Arno Pauly:

Weihrauch degrees of finding equilibria in sequential games

15:30 Andrej Bauer and Kazuto Yoshimura: The Weihrauch lattice is too small

16:00 Kazuto Yoshimura:

Same for Bishop, different for Weihrauch

16:30 Coffee

17:00 Tahina Rakotoniaina and Vasco Brattka: A survey on the strength of Ramseys Theorem

17:30 Robert Lubarsky and Matt Hendtlass: Separating Variants of LEM, LPO, and MP 
July 23rd (Wednesday) Morning: Special Session on Implementation of Exact Real Number Arithmetic

09:00 Keith Briggs:

Computational aspects of simultaneous Diophantine approximation

09:30 Valérie Ménissier-Morain:

Tools for a gentle slope transition from floating point arithmetic to exact real arithmetic

10:00 Gregorio de Miguel Casado:

Decimal on-line Arithmetic for Rational Operation in $\mathrm{C}++($ DARIO $\mathrm{C}++)$

10:30 Coffee

11:00 Michal Konečný and Jan Duracz:

Exact Function Interval Arithmetic

11:30 Franz Brauße, Margarita Korovina, Norbert Th. Müller and Alexander van Ackerern:

Exact real arithmetic and ODE systems with polynomial right hand sides

12:00 Akitoshi Kawamura, Florian Steinberg and Holger Thies: Analytic Functions in iRRAM

12:30 Lunch

14:00 Excursion \& Dinner:

Guided tour of Grube Messel, UNESCO world heritage site due to its vast collection of fossiles, followed by ...

a vegetarian dinner served in TU Darmstadt's Georg-

Christoph-Lichtenberg-Haus
July 24th (Thursday) Morning Session:

Dynamical Systems, ODEs/PDEs, Complex Analysis

09:00 Christoph Spandl:

True orbit simulations of dynamical systems for validating molecular dynamics simulations

09:30 Michal Konečný, Jan Duracz, Amin Farjudian and Walid Taha:

Picard Method for Enclosing ODEs with Uncertain Initial Values

10:00 Pieter Collins:

Computability of Solutions of Stochastic Differential Equations

10:30 Svetlana Selivanova, Victor Selivanov:

On computability of boundary-value problems for some linear hyperbolic PDEs

11:00 Coffee

11:30 Timothy McNicholl (Invited Talk):

Explorations of effective local connectivity

12:30 Alexander Melnikov (Invited Talk):

Computable Metric Space Theory is a Generalization of Effective Algebra

13:30 Closing Address 


\section{Table of Contents}

\section{Invited papers}

Characterizations of the topological complexity of discontinuous functions ... Matthew de Brecht

A constructive manifestation of the Kleene-Kreisel continuous functionals ... Martín Escardó and Chuangjie Xu

Techniques in randomness and ergodic theory $\ldots \ldots \ldots \ldots \ldots \ldots \ldots$ Johanna Franklin

Explorations of effective local connectivity $\ldots \ldots \ldots \ldots \ldots \ldots \ldots \ldots \ldots \ldots \ldots$ Timothy McNicholl

Computable Metric Space Theory is a Generalization of Effective Algebra ... Alexander Melnikov

The descriptive theory of represented spaces $\ldots \ldots \ldots \ldots \ldots \ldots \ldots$ Arno Pauly

\section{Contributed papers}

On computability and disintegration $\ldots \ldots \ldots \ldots \ldots \ldots \ldots \ldots \ldots \ldots \ldots \ldots \ldots \ldots \ldots$ Nathanael Ackerman, Cameron Freer and Daniel Roy

The Weihrauch lattice is too small $\ldots \ldots \ldots \ldots \ldots \ldots \ldots \ldots \ldots \ldots \ldots$ Andrej Bauer and Kazuto Yoshimura

Probabilistic Computability and Choice................. Vasco Brattka, Guido Gherardi and Rupert Hölzl

Exact real arithmetic and ODE systems with polynomial right hand sides ... Franz Brauße, Margarita Korovina, Norbert Th. Müller and Alexander van Ackerern

Computational aspects of simultaneous Diophantine approximation . . . . . . Keith Briggs

Computability of Solutions of Stochastic Differential Equations . . . . . . . . . Pieter Collins

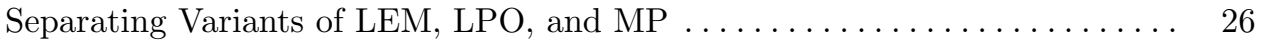
Matt Hendtlass and Robert Lubarsky

Computable transition points . . . . . . . . . . . . . . . Zvonko Iljazović and Bojan Pažek

Analytic Functions in iRRAM ....................... Akitoshi Kawamura, Florian Steinberg and Holger Thies

On the Computational Power of Algorithmically Random Constants in

Blum-Shub-Smale Machines ....................... Akitoshi Kawamura and Kentarô Yamamoto

On the effectively $\mathrm{G}_{\delta}$-decomposable functions Takayuki Kihara 
Exact Function Interval Arithmetic . . . . . . . . . . . . . . . . . . 39

Michal Konečný and Jan Duracz

Picard Method for Enclosing ODEs with Uncertain Initial Values . . . . . . . 41 Michal Konečný, Jan Duracz, Amin Farjudian and Walid Taha

Tools for a gentle slope transition from floating point arithmetic to exact

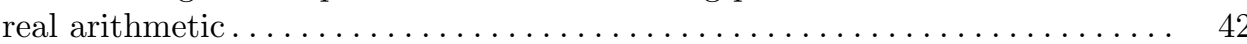
Valérie Ménissier-Morain

Decimal on-line Arithmetic for RatIonal Operation in C++ (DARIO C++) . 45 Gregorio de Miguel Casado

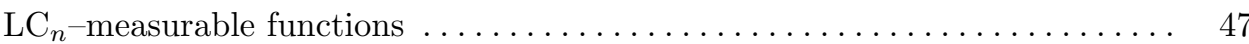
Alexey Ostrovsky

Computability for Basic Quantum Mechanics based on the Hilbert Lattice . . 49 Martin Pape

A survey on the strength of Ramsey's Theorem ................. 51 Tahina Rakotoniaina and Vasco Brattka

Weihrauch degrees of finding equilibria in sequential games ............ 52 Stéphane Le Roux and Arno Pauly

Characterising classes of hyperprojective qcb-spaces ............... 54 Matthias Schröder

True orbit simulations of dynamical systems for validating molecular

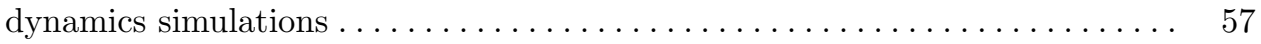
Christoph Spandl

Strong properness of dyadic subbases ...................... 58 Yasuyuki Tsukamoto and Hideki Tsuiki

Same for Bishop, different for Weihrauch . . . . . . . . . . . . . . 60 Kazuto Yoshimura

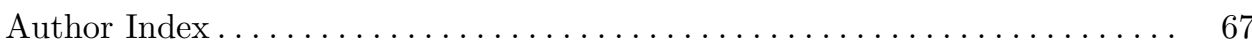

\title{
Program Committee
}

\author{
Vasco Brattka \\ Mathieu Hoyrup \\ Zvonko Iljazović \\ Takayuki Kihara \\ Ulrich Kohlenbach \\ Elvira Mayordomo \\ Joseph S. Miller \\ Norbert Müller \\ Daniel Roy
}




\title{
Characterizations of the topological complexity of discontinuous functions*
}

\author{
Matthew de Brecht \\ CiNet, NICT, Osaka, Japan
}

It is well known in computable analysis that discontinuous functions on admissibly represented topological spaces are not computable for simple information theoretic reasons. When working with such functions, the user must either provide additional information about the input, or else be satisfied with a weaker representation of the output (such as a sequence of points that converge to the correct output with some unknown modulus of convergence). There has been a great deal of work characterizing the degree of discontinuous functions, which has led to deep connections with descriptive set theory and various notions of limit computability.

Within the framework of Type Two Theory of Effectivity, several researchers such as V. Brattka and M. Ziegler have investigated certain "jump operators" which modify the representation of a space and can capture classes of discontinuous functions and limit computability. Here we investigate a general definition of "jump operator" in an attempt to unify this line of research. We will show that some common notions of limit computability can be reinterpreted as jump operators, and we will also provide topological characterizations of the functions that are realizable in this way. We will also show that our approach adds a nice category theoretical perspective to the analysis of degrees of discontinuity.

A represented space is a pair $\langle X, \rho\rangle$ where $X$ is a set and $\rho: \subseteq \omega^{\omega} \rightarrow X$ is a surjective partial function. If $\mathbf{X}=\left\langle X, \rho_{X}\right\rangle$ and $\mathbf{Y}=\left\langle Y, \rho_{Y}\right\rangle$ are represented spaces and $f: \subseteq X \rightarrow Y$ is a partial function, then a function $F: \subseteq \omega^{\omega} \rightarrow \omega^{\omega}$ realizes $f$ if and only if $f \circ \rho_{X}=\rho_{Y} \circ F$. We say that a function between represented spaces is (continuously) realizable if it is realized by some continuous function.

Definition 1. A (topological) jump operator is a partial surjective function $j: \subseteq \omega^{\omega} \rightarrow \omega^{\omega}$ such that for every partial continuous $F: \subseteq \omega^{\omega} \rightarrow \omega^{\omega}$, there is partial continuous $F^{\prime}: \subseteq \omega^{\omega} \rightarrow \omega^{\omega}$ such that $F \circ j=j \circ F^{\prime}$.

A $j$-realizer of a function $f$ between the represented spaces $\mathbf{X}$ and $\mathbf{Y}$ is a function $F: \subseteq \omega^{\omega} \rightarrow \omega^{\omega}$ such that $f \circ \rho_{X}=\rho_{Y} \circ j \circ F$. In other words, $F$ is a $j$-realizer of $f$ if and only if $F$ realizes $f$ when we reinterpret the codomain of

\footnotetext{
*A full length paper with references is available online: M. de Brecht (2013): Levels of discontinuity, limit-computability, and jump operators. arXiv:1312.0697.
} 
$f$ to be $j(\mathbf{Y}):=\left\langle Y, \rho_{Y} \circ j\right\rangle$, the " $j$-jump" of $\mathbf{Y}$. We will say that a function is $j$-realizable if and only if it has a continuous $j$-realizer.

The following jump operators are of particular interest to computable analysis (we let $\langle\cdots\rangle_{n \in \omega}:\left(\omega^{\omega}\right)^{\omega} \rightarrow \omega^{\omega}$ be some fixed computable bijection):

- Define $j_{\mathbf{\Sigma}_{2}^{0}}: \subseteq \omega^{\omega} \rightarrow \omega^{\omega}$ as:

$$
\begin{aligned}
\left\langle\xi_{n}\right\rangle_{n \in \omega} \in \operatorname{dom}\left(j_{\boldsymbol{\Sigma}_{2}^{0}}\right) & \Leftrightarrow \xi_{0}, \xi_{1}, \ldots \text { converges in } \omega^{\omega} \\
j_{\boldsymbol{\Sigma}_{2}^{0}}\left(\left\langle\xi_{n}\right\rangle_{n \in \omega}\right) & =\lim _{n \in \omega} \xi_{n}
\end{aligned}
$$

For each natural number $n>1$ define $j_{\boldsymbol{\Sigma}_{n+1}^{0}}=j_{\boldsymbol{\Sigma}_{n}^{0}} \circ j_{\boldsymbol{\Sigma}_{2}^{0}}$.

- Define $j_{\Delta}: \subseteq \omega^{\omega} \rightarrow \omega^{\omega}$ as:

$$
\begin{aligned}
\left\langle\xi_{n}\right\rangle_{n \in \omega} \in \operatorname{dom}\left(j_{\Delta}\right) & \Leftrightarrow(\exists n)(\forall m \geq n)\left[\xi_{m}=\xi_{n}\right] \\
j_{\Delta}\left(\left\langle\xi_{n}\right\rangle_{n \in \omega}\right) & =\lim _{n \in \omega} \xi_{n}
\end{aligned}
$$

- For each countable ordinal $\alpha$, define $j_{\alpha}: \subseteq \omega^{\omega} \rightarrow \omega^{\omega}$ as:

$$
\begin{aligned}
\left\langle\left\langle\beta_{n}\right\rangle_{\alpha} \diamond \xi_{n}\right\rangle_{n \in \omega} \in \operatorname{dom}\left(j_{\alpha}\right) \Leftrightarrow & (\forall n)\left(\alpha>\beta_{n} \geq \beta_{n+1}\right) \text { and } \\
& (\forall n)\left(\xi_{n} \neq \xi_{n+1} \Rightarrow \beta_{n} \neq \beta_{n+1}\right) \\
j_{\alpha}\left(\left\langle\left\langle\beta_{n}\right\rangle_{\alpha} \diamond \xi_{n}\right\rangle_{n \in \omega}\right)= & \lim _{n \in \omega} \xi_{n}
\end{aligned}
$$

where $\langle\cdot\rangle_{\alpha}: \alpha \rightarrow \omega$ is some fixed encoding of ordinals less than $\alpha$ as natural numbers, and $\langle\beta\rangle_{\alpha} \diamond \xi$ is the element of $\omega^{\omega}$ obtained by prepending the encoding of $\beta$ to the beginning of $\xi$.

Realizability according to these jump operators is best understood in terms of "limit-computability" by Type Two Turing machines (possibly with access

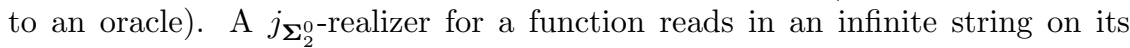
input tape and produces an infinite string on the output tape, but is allowed to modify the contents of each cell in the output tape a finite number of times. A $j_{\Delta}$-realizer is similar, but it is only allowed to make finitely many revisions (so after some finite amount of time a $j_{\Delta}$-realizer will no longer make revisions, but it is possible for a $j_{\boldsymbol{\Sigma}_{2}^{0}}$-realizer to make infinitely many revisions so long as it only revises each particular cell a finite number of times). A $j_{\alpha}$-realizer outputs an (encoding of an) ordinal less than $\alpha$, and must decrement its ordinal bound every time it makes a revision without ever going below zero.

For functions between admissibly represented countably based $T_{0}$-spaces, the realizability notions induced by these jump operators have the following topological characterizations:

- A function is $j_{\boldsymbol{\Sigma}_{n}^{0}}$-realizable $(1 \leq n<\omega)$ if and only if it is $\boldsymbol{\Sigma}_{n}^{0}$-measurable. This was first observed for metric spaces by V. Brattka and M. Ziegler. 
- A function is $j_{\Delta}$-realizable if and only if it is $\boldsymbol{\Delta}_{2}^{0}$-piecewise continuous. This was first observed for metric spaces by A. Andretta, and the characterization is important for analyzing classical descriptive set theory results like the Jayne-Rogers theorem.

- A function is $j_{\alpha}$-realizable $\left(\alpha<\omega_{1}\right)$ if and only if its level of discontinuity is $\alpha$ according to $\mathrm{P}$. Hertling's hierarchy. This characterization appears to be new, but L. Motto Ros has independently investigated a similar notion of realizability for metric spaces. It is also related to previous work on the connections between the Hausdorff difference hierarchy, Cantor-Bendixson rank, and computation with ordinal mind-change bounds.

The above results show that jump operators provide a unifying approach to classifying the degree of discontinuity of functions between countably based $T_{0}$-spaces.

Next we switch to a category theoretic perspective. The definition of a (topological) jump operator given above is chosen so that taking the $j$-jump, i.e. the mapping $\left\langle Y, \rho_{Y}\right\rangle \mapsto\left\langle Y, \rho_{Y} \circ j\right\rangle$, is functorial within the category of represented spaces and continuously realizable functions. When dealing with the category of computably realizable functions, the definition of a jump operator must be modified so that $F$ and $F^{\prime}$ range over all partial computable functions. This distinction is necessary, because V. Brattka, A. Pauly, and the author showed that in the effective category there is a jump operator that is left adjoint to $j_{\boldsymbol{\Sigma}_{2}^{0}}$ and which fails to be a jump operator in the category of continuously realizable functions (it is interesting to note that the monad from this adjoint is a jump operator in the effective category that characterizes the recursion theoretic notion of low-computability).

Since the $j$-realizable functions from $\mathbf{X}$ to $\mathbf{Y}$ are precisely the realizable functions from $\mathbf{X}$ to $j(\mathbf{Y})$, the cartesian closedness of the category of represented spaces and realizable functions allows a natural interpretation of the exponential object $j(\mathbf{Y})^{\mathbf{X}}$ as being the space of $j$-realizable functions from $\mathbf{X}$ to $\mathbf{Y}$. In particular, if we let $\Sigma$ be the Sierpinski space with an admissible representation, then the object $j(\Sigma)^{\mathbf{X}}$ is the space of " $j$-semi-decidable" subsets of $\mathbf{X}$. In particular, if $\mathbf{X}$ is an admissibly represented countably based $T_{0}$-space, then $j_{\boldsymbol{\Sigma}^{0}}(\Sigma)^{\mathbf{X}}$ is the space of $\boldsymbol{\Sigma}_{n}^{0}$-subsets of $\mathbf{X}$.

Certain abstract re-formulations of topology, such as Abstract Stone Duality and Synthetic Topology, identify open subsets of an arbitrary object with functions into a Sierpinski-like object. Basic topological properties such as being compact, overt, Hausdorff, or discrete, are defined and applied in such frameworks using diagrams involving the Sierpinski space $\Sigma$. Within the category of represented spaces and realizable functions, for any jump operator $j$ we can simply replace $\Sigma$ with $j(\Sigma)$ and obtain relativized definitions of $j$-compact, $j$ overt, $j$-Hausdorff, and $j$-discrete. Concepts such as overtness, which is trivial in frameworks based on classical logic, often become highly non-trivial (even classically) when relativized to most jump operators. This leads to many questions about the relationship between abstract approaches to topology, descriptive set theory, computability theory, and the use of modal operators in type theories. 


\title{
A constructive manifestation of the Kleene-Kreisel continuous functionals
}

\author{
Martín Escardó and Chuangjie Xu \\ University of Birmingham, UK
}

\begin{abstract}
We identify yet another category equivalent to that of KleeneKreisel continuous functionals. Reasoning constructively and predicatively, all functions from the Cantor space to the natural numbers are uniformly continuous in this category. We do not need to assume Brouwerian continuity axioms to prove this, but, if we do, then we can show that the full type hierarchy is equivalent to our manifestation of the continuous functionals. We construct this manifestation within a category of concrete sheaves, called C-spaces, which form a locally cartesian closed category, and hence can be used to model system $\mathrm{T}$ and dependent types. We show that this category has a fan functional and validates the uniform continuity axiom in these theories. Our development is within informal constructive mathematics, along the lines of Bishop mathematics. However, in order to extract concrete computational content from our constructions, we formalized it in intensional Martin-Löf type theory, in Agda notation.
\end{abstract}

Technical summary. In a cartesian closed category with a natural numbers object $\mathbb{N}$, define the simple objects to be the least collection containing $\mathbb{N}$ and closed under exponentials (function spaces). The simple objects of any such category give an interpretation of the simply typed lambda calculus and higher-type primitive recursion (the term language of Gödel's system T). The Kleene-Kreisel continuous functionals, or countable functionals [13], form a category equivalent to the full subcategory on the simple objects of any of the following categories, among others: (1) compactly generated topological spaces [13,6], (2) sequential topological spaces [6], (3) Simpson and Schröder's QCB spaces [2, 6], (4) Kuratowski limit spaces [7], (5) filter spaces [7], (6) Scott's equilogical spaces [3], (7) Johnstone's topological topos [9]. See Normann [14] and Longley [10, 11] for the relevance of Kleene-Kreisel spaces in the theory of higher-type computation. Counter-examples include Hyland's effective topos [8] and the hereditary effective operations (HEO) [10], which give a second simple-type hierarchy [10]. A third type hierarchy, discussed here in connection with the continuous functionals, is the full type hierarchy, which is the full subcategory on the simple objects of the category of sets [13].

We work with a category of sheaves, analogous to the topological topos, and 
with a full subcategory of concrete sheaves [1], here called C-spaces, analogous to the limit spaces. The $\mathrm{C}$-spaces can be described as sets equipped with a suitable continuity structure, and their natural transformations can be regarded as continuous maps. The main contributions of this work are the following:

1. The simple C-spaces form a category equivalent to that of Kleene-Kreisel continuous functionals.

The proof here is non-constructive (as are the proofs of the above equivalences). But we claim that the C-spaces form a good substitute of the above categories of spaces for the purposes of constructive reasoning.

2. If we assume the Brouwerian axiom that all set-theoretic functions $\mathbf{2}^{\mathbb{N}} \rightarrow \mathbb{N}$ are uniformly continuous, then we can show constructively that the full type hierarchy is equivalent to the Kleene-Kreisel continuous hierarchy within C-spaces.

3. Without assuming Brouwerian axioms, we show constructively that the category of C-spaces has a fan functional $\left(\mathbf{2}^{\mathbb{N}} \rightarrow \mathbb{N}\right) \rightarrow \mathbb{N}$ that continuously calculates moduli of uniform continuity of maps $2^{\mathbb{N}} \rightarrow \mathbb{N}$.

4. C-Spaces give a model of system $\mathrm{T}$ with a uniform continuity axiom, expressed as the skolemization of

$$
\forall f: \mathbf{2}^{\mathbb{N}} \rightarrow \mathbb{N} . \exists m \in \mathbb{N} . \forall \alpha, \beta \in \mathbf{2}^{\mathbb{N}} \cdot \alpha={ }_{m} \beta \Longrightarrow f \alpha=f \beta,
$$

where $\alpha={ }_{m} \beta$ stands for $\forall i<m . \alpha_{i}=\beta_{i}$, with the aid of a fan-functional constant.

5. C-Spaces give a model of dependent types with a uniform continuity axiom, expressed as a type via the Curry-Howard interpretation:

$$
\prod_{f: \mathbf{2}^{\mathbb{N}} \rightarrow \mathbb{N}} \sum_{m \in \mathbb{N}} \prod_{\alpha, \beta \in \mathbf{2}^{\mathbb{N}}}\left(\alpha={ }_{m} \beta \Longrightarrow f \alpha=f \beta\right) .
$$

6. We give a constructive treatment of $\mathrm{C}$-spaces suitable for development in a predicative intuitionistic type theory in the style of Martin-Löf, which we formalized in Agda notation [12, 5] for concrete computational purposes.

We stress, however, that in this talk we deliberately reason informally, along the lines of Bishop mathematics [4].

As mentioned above, our sheaf topos is closely related to Johnstone's topological topos. Working non-constructively, one can show that the topological topos has a fan functional and that it interprets uniform continuity axioms for both simple and dependent types. The point of our contribution is that we can achieve this by working constructively instead, without assuming Brouwerian axioms, and remaining predicative. 


\section{References}

[1] J. C. Baez and A. E. Hoffnung. Convenient categories of smooth spaces. Transactions of the American Mathematical Society, 363(11):5789-5825, 2011.

[2] I. Battenfeld, M. Schröder, and A. Simpson. Compactly generated domain theory. Mathematical Structures in Computer Science, 16(2):141-161, 2006.

[3] A. Bauer, L. Birkedal, and D. S. Scott. Equilogical spaces. Theoretical Computer Science, 315(1):35-59, 2004.

[4] E. Bishop and D. Bridges. Constructive analysis, volume 279. SpringerVerlag, 1985.

[5] A. Bove and P. Dybjer. Dependent types at work. Proceedings of Language Engineering and Rigorous Software Development, LNCS, 5520:57-99, 2009.

[6] M. H. Escardó, J. Lawson, and A. Simpson. Comparing cartesian closed categories of (core) compactly generated spaces. Topology Appl., 143(13):105-145, 2004.

[7] J. M. E. Hyland. Filter spaces and continuous functionals. Annals of Mathematical Logic, 16:101-143, 1979.

[8] J. M. E. Hyland. The effective topos. In The L.E.J. Brouwer Centenary Symposium (Noordwijkerhout, 1981), volume 110 of Studies in Logic and the Foundations of Mathematics, pages 165-216. North-Holland, 1982.

[9] P. T. Johnstone. On a topological topos. Proceedings of the London Mathematical Society, 38(3):237-271, 1979.

[10] J. R. Longley. Notions of computability at higher types. I. In Logic Colloquium 2000, volume 19 of Lecture Notes in Logic, pages 32-142. The Association for Symbolic Logic, 2005.

[11] J. R. Longley. On the ubiquity of certain total type structures. Mathematical Structures in Computer Science, 17(5):841-953, 2007.

[12] U. Norell. Dependently typed programming in agda. In Proceedings of the 4 th international workshop on Types in language design and implementation, TLDI '09, pages 1-2, 2009.

[13] D. Normann. Recursion on the countable functionals, volume 811 of Lecture Notes in Mathematics. Springer, 1980.

[14] D. Normann. Computing with functionals - computability theory or computer science? The Bulletin of Symbolic Logic, 12(1):43-59, 2006. 


\title{
TECHNIQUES IN RANDOMNESS AND ERGODIC THEORY
}

\author{
JOHANNA N.Y. FRANKLIN \\ UNIVERSITY OF CONNECTICUT
}

A point is algorithmically random if it is typical with respect to some class of measure-zero computability-theoretic properties. Clearly, different computability requirements for these properties generate different classes of random points. Ergodic theorems describe typicality with respect to ergodic (or simply measure-preserving) transformations: a typical ergodic theorem states that, given any transformation of a certain kind, the orbit of almost every point in a space behaves regularly. It is natural to ask whether, when an ergodic theorem is effectivized in a particular way, the points that satisfy this ergodic theorem are precisely the points that are algorithmically random with respect to some class of properties.

This topic has been studied extensively in recent years, and it has been found that the above question can often be answered in the affirmative, regardless of the way in which the ergodic theorem is effectivized. In this talk, I'll survey the techniques that have been used in the proofs of these results, focusing on my own work with Towsner and the method of cutting and stacking [1].

\section{REFERENCES}

[1] Johanna N.Y. Franklin and Henry Towsner. Randomness and non-ergodic systems. Mosc. Math. J. To appear. 


\section{Explorations of effective local connectivity}

Timothy H. McNicholl, Ph.D.

Department of Mathematics

Iowa State University

A topological space $X$ is locally connected if, whenever $U$ is a neighborhood of a point $p$ of $X, U$ contains a connected neighborhood $V$ that contains $p$. We will discuss:

- Several natural ways to define effective notions of local connectivity and their equivalence.

- Applications to computable space-filling curves.

- Applications to boundary extensions of computable conformal maps. 


\title{
COMPUTABLE METRIC SPACE THEORY IS A GENERALIZATION OF EFFECTIVE ALGEBRA
}

\author{
ALEXANDER MELNIKOV
}

In my CCA 2014 talk I will concentrate on several recent applications of effective algebra to computable metric and Banach spaces. It is clear that both fields have very similar underlying ideas and motivation. For instance, in both fields we ask:

- what does it mean for an (algebraic/metric) structure to be algorithmically presented?

- which (algebraic/analytic) operations on the structure are effective?

- how many effective presentations does a structure have up to computable isomorphism?

- can we classify effectively presented members of a given class?

- can we measure the complexity of an effective classification problem?

and other familiar questions. Despite of these obvious similarities, the fields have been developing almost independently. I will survey several recent projects that aim to (re)unite these fields both technically and philosophically ${ }^{1}$.

0.1. A reduction. We will discuss a rather elementary proof of the following observation (due to Khoussainov and myself):

Fact. There exists an injective effective functor $\Psi$ from the class of computable algebraic structures to the class of computable metric spaces that preserves all structural and effective features.

In other words, every algebraic structure can be effectively "coded" into a metric space and then, if necessary, effectively "uncoded" back. Furthermore, $A \cong{ }^{c o m p} B$ iff $\Psi(A) \cong{ }_{i s o}^{c o m p} \Psi(B)$. Indeed, the functor can be modified to range over perfect Polish spaces. The fact above has several interesting corollaries and also a peculiar philosophical interpretation stated in the title.

0.2. The right category. In effective algebra, each countable algebra (e.g., a group) corresponds to a class of classically isomorphic computable presentations that are typically viewed up to computable isomorphisms. We associate each computably presentable metric or Banach space with a class of computable countable algebraic structures under a specific effective morphism that will be defined. This approach will allow us to derive several interesting adaptations of effective algebraic techniques to computable metric and Banach space theory.

\footnotetext{
${ }^{1}$ Based on papers jointly written with Ng, Greenberg, Turetsky, and Nies.
} 
0.3. The right language. In effective algebra definability plays a central role. A classical result of effective algebra states that an operation is computably enumerable in every presentation of an algebra if and only if it is $\Sigma_{1}$-definable in the language of the algebra. We choose an appropriate language and extend this and similar results to effective metric and Banach space theory. We will also discuss several pleasant applications of the result to classical problems that go back to Pour-El and Richards.

0.4. Index sets and higher categoricity. In recursion theory and effective algebra one typically uses hyperarithmetical hierarchy and index sets to measure the complexity of a classification problem. Another closely related approach uses isomorphisms that are computable relative to an oracle. We apply these ideas to obtain several classification-type results for compact metric and probability spaces.

0.5. Questions. I conclude my talk with a short list of selected open problems.

UC AT BERKELEY

E-mail address: alexander.g.melnikov@gmail.com 


\title{
The descriptive theory of represented spaces
}

\author{
Arno Pauly \\ Clare College \\ University of Cambridge, United Kingdom \\ Arno.Pauly@cl.cam.ac.uk
}

The emergent descriptive theory of represented spaces encompasses several recent developments and research projects that are to be summarized here. It has been demonstrated by DE BRECHT [1] and others that many results from classic descriptive set theory can be extended from Polish spaces to to Quasi-Polish spaces. Beyond a mere extension of the known theory though, we will see that the larger scope allows us to introduce entirely new techniques.

The category of represented spaces has sufficient closure properties to form all relevant derived spaces considered in descriptive set (and function) theory. Moreover, as shown by the author and DE BRECHT [7], these constructions can be expressed in terms of endofunctors - and doing so both provides avenues for simple proofs and a deeper understanding of theorems such as the Jayne-Rogers theorem [8].

Taking into account explicit the representations of spaces exposes the connection between dimension-theoretic properties of spaces and certain substructures of the Medvedev degrees. Building partially on prior work by MILLER [5], KIHARA and the author presented and exploited this connection in [4].

Further work to be touched upon is SCHRÖDER's and SELIVANOV's study of represented spaces with certain complexity of the equivalence relation [10, 9]; KIHARA's work on a computable decomposition theorem [3]; the agreement of notions of synthetic descriptive set theory with the approach in effective descriptive set theory demonstrated by KISPETÉR, GreGORIADES and the author; and the translation of Mотто-Ros' constructions of games for pointclasses [6] into the language of Weihrauch reducibility by NoBREGA [2].

\section{References}

[1] Matthew de Brecht (2013): Quasi-Polish spaces. Annals of Pure and Applied Logic 164(3), pp. $354-381$.

[2] Hugo de Holanda Cunha Nobrega (2013): Game characterizations of function classes and Weihrauch degrees. M.Sc. thesis, University of Amsterdam.

[3] Takayuki Kihara (2013). Decomposing Borel functions using the Shore-Slaman join theorem. arXiv 1304.0698.

[4] Takayuki Kihara \& Arno Pauly (2014). Point degree spectra of represented spaces. arXiv:1405.6866.

[5] Joseph S. Miller (2004): Degrees of Unsolvability of Continuous Functions. Journal of Symbolic Logic 69(2), pp. $555-584$.

[6] Luca Motto Ros (2011): Game representations of classes of piecewise definable functions. Mathematical Logic Quarterly 57(1), pp. 95-112.

[7] Arno Pauly \& Matthew de Brecht. Towards Synthetic Descriptive Set Theory: An instantiation with represented spaces. arXiv 1307.1850. 
[8] Arno Pauly \& Matthew de Brecht (2014): Non-deterministic Computation and the Jayne Rogers Theorem. Electronic Proceedings in Theoretical Computer Science 143. DCM 2012.

[9] Matthias Schröder \& Victor Selivanov (2014). Hyperprojective Hierarchy of QCB $B_{0}$-spaces. arXiv 1404.0297. Available at http://arxiv.org/abs/1404.0297.

[10] Matthias Schröder \& Victor L. Selivanov (2013). Some hierarchies of QCB $B_{0}$-spaces. arXiv 1304.1647. Available at http://arxiv.org/abs/1304.1647. 


\title{
Extended abstract: On computability and disintegration
}

\author{
Nathanael L. Ackerman ${ }^{1}$, Cameron E. Freer ${ }^{2}$, and Daniel M. Roy ${ }^{3}$ \\ ${ }^{1}$ Department of Mathematics, Harvard University \\ ${ }^{2}$ Massachusetts Institute of Technology and Analog Devices Lyric Labs \\ ${ }^{3}$ Department of Engineering, University of Cambridge
}

Conditioning is a basic tool in probability theory that can be understood in several different ways. While conditioning is often characterized in terms of averaging, or in terms of projection, the perspective most often taken in statistical contexts is that of disintegration. One of the primary goals of Bayesian statistical inference is to compute or summarize the disintegration of a collection of random variables (or their joint distribution) with respect to a subfamily of "observed" random variables. On the other hand, disintegrations are defined only up to null sets, and so their evaluation at points, as is statistical practice, has typically relied upon additional (sometimes unstated) hypotheses.

One such hypothesis is the continuity of some version of the disintegration, which ensures that it is canonically defined everywhere in the support of the distribution of the conditioning variables, and it is interesting to consider the computability of the conditioning operator in this context.

In [AFR11] and [AFR10], we showed that disintegrations of computable random variables need not be computable on any measure one (or indeed any positive measure) set, even when there is guaranteed to be a continuous version. Here we strengthen and uniformize these results, by providing precise bounds on how noncomputable disintegration can be. We make use of certain constructive definitions of disintegration, especially those of Tjur; see [Tju75] and [Tju80]. Other work in this direction includes [Pfa79], [FMNP95], and [Zab79].

We show that the disintegration operator on topological spaces along a projection map, restricted to measures for which the disintegration is continuous, is strongly Weihrauch-equivalent to the limit operator Lim (for definitions, see below). We also show that in the case where the disintegration exists everywhere (but is not necessarily continuous), the disintegration itself is strongly Weihrauch-reducible to Lim, and further exhibit a single distribution realizing this upper bound.

A result by Hoyrup, Rojas, and Weihrauch [HRW12] (see also [HR11]) can be shown to imply that the map taking a distribution and a real $\varepsilon>0$ to a continuous disintegration on some $(1-\varepsilon)$-measure set is Weihrauch reducible to the limit operator Lim. Our work can therefore also be viewed as an extension of this result.

Tjur points and disintegrations. We briefly describe Tjur points, which Tjur used in order to provide a constructive definition of disintegrations in certain cases; for more details, see [Tju80, §9.7].

We assume that every topological space is Hausdorff, locally finite, and inner regular, and that its underlying set along with the collection of all its Borel subsets is a standard Borel space; in this case all measures on such a space are Radon. The following property is due to Tjur, and is a slight rearrangement of a property described in [Tju75].

Definition 1 (Tjur Property). Let $S$ and $T$ be topological spaces, let $\mu$ be a Borel probability measure on $S$, and let $g: S \rightarrow T$ be a measurable function. Suppose $x \in T$ is such that for every open neighborhood $U$ of $x$, we have $\mu\left(g^{-1}(U)\right)>0$. Let $\mathscr{D}(x)$ denote the set of pairs $(U, B)$ where $U$ is an open neighborhood of $x$ and $B$ is a measurable subset of $U$ with $\mu\left(g^{-1}(B)\right)>0$. We write $(U, B) \preccurlyeq\left(U^{\prime}, B^{\prime}\right)$ when $U^{\prime} \supseteq U$. Note that this relation is a partial ordering on $\mathscr{D}(x)$ and makes $\mathscr{D}(x)$ a directed set. We say that $x$ has the Tjur property (for $\mu$ along $g$ ) when the directed limit $\mu_{g}^{x}(\cdot):=\lim _{(U, B) \in \mathscr{D}(x)}\left(\mu \circ g^{-1}\right)^{B}(\cdot)$ exists and is a probability measure, where the superscript denotes the conditional probability with respect to $B$.

Many common properties imply that a point is Tjur. For example, every point of continuity of an absolutely continuous distribution is a Tjur point. Also, any isolated point mass (e.g., a point in the 
support of a discrete random variable taking values in a discrete space) is a Tjur point. On the other hand, nonisolated point masses are not necessarily Tjur points.

The following lemma is a consequence of Corollary 9.9.2 and Proposition 9.10.1 of [Tju80]. For more details on the notion of disintegration, see [Kal02, §6].

Lemma 2. Let $S$ be a topological space, $T$ a metric space, $\mu$ a Borel probability measure on $S$, and $g: S \rightarrow T$ a measurable function. Suppose that $\left(\mu \circ g^{-1}\right)$-almost all $x \in T$ have the Tjur property (for $\mu$ along g). For each such Tjur point $x$, suppose that $\left\{B_{n}^{x}\right\}_{n \in \mathbb{N}}$ is a sequence of measurable sets for which each $B_{n}^{x}$ is contained in the $2^{-n}$-ball around $x$ and $\mu\left(g^{-1}\left(B_{n}^{x}\right)\right)>0$.

Then the function $\kappa: S \times \mathcal{B}_{T} \rightarrow[0,1]$ given by $\kappa(x, A):=\lim _{n \rightarrow \infty} \mu^{B_{n}^{x}}(A)$ for Tjur points $x$ and Borel sets $A \subseteq T$ (and defined by $\kappa(x, \cdot)=\nu$ for an arbitrary probability measure $\nu$ otherwise) is a version of the disintegration of $\mu$ along $g$.

Even when such limits exist, they may not be computable. One of the main constructions of [AFR10], which we generalize here, is an example of a conditional distribution for which almost all points are Tjur, and yet no version of the disintegration is a computable map.

Given a topological space $S$, write $M_{1}(S)$ for the topological space of probability measures on $S$ under the weak topology, and $\delta_{M_{1}(S)}$ for its representation. For topological spaces $S$ and $T$, let $\mathscr{C}_{S, T} \subseteq M_{1}(S \times T)$ be the subset consisting of those measures $\mu$ that admit a continuous disintegration and that have full measure, in the sense that $\mu(S \times A)>0$ for every nonempty open $A \subseteq T$. Let $\delta_{\mathscr{C}_{S, T}}$ be the restriction of the representation $\delta_{M_{1}(S \times T)}$ to $\mathscr{C}_{S, T}$.

Let $C\left(T, M_{1}(S)\right)$ be the class of continuous functions from $T$ to $M_{1}(S)$, and define the map $\widehat{\mathrm{D}}_{S, T}: \mathscr{C}_{S, T} \rightarrow C\left(T, M_{1}(S)\right)$ by $\widehat{\mathrm{D}}_{S, T}(\mu):=f$ such that $\mu(A \times B)=\int_{B}(f(t))(A) \mu(S \times d t)$. Note that this is well-defined by the definition of $\mathscr{C}_{S, T}$. Also observe that $\kappa$, as defined in Lemma 2 , is equal to $\widehat{\mathrm{D}}_{S, T}(\mu)$ when it is a.e. continuous. The following lemma is a consequence of Proposition 9.14.2 of [Tju80].

Lemma 3. Let $S$ and $T$ be topological spaces and let $\mu$ be a Borel probability measure on $S \times T$. Suppose that every point of $T$ is a Tjur point for $\mu$ along the projection map. Then the disintegration $\widehat{\mathrm{D}}_{S, T}(\mu): T \rightarrow M_{1}(S)$ is continuous.

We make use of Lemmas 2 and 3 to prove the continuity of a particular disintegration, which is a key step in establishing a lower-bound in the Weihrauch degrees for the disintegration operator, in the proof of Proposition 4.

Weihrauch reducibility and represented spaces. For background on Weihrauch reducibility, Weihrauch degrees, and represented spaces, see, e.g., [BG11], [Pau12], or the introduction to [BHG13]. Recall the Lim (on Cantor space) and EC operators, which are equivalent by [Bra05, Prop. 9.1].

For computable topological spaces $S$ and $T$, define the disintegration operator $D_{S, T}: \subseteq \mathbb{N}^{\mathbb{N}} \rightrightarrows \mathbb{N}^{\mathbb{N}}$ to be the partial multi-map taking any representation of a measure $\mu \in \mathscr{C}_{S, T}$ to the representations of its disintegration $\widehat{\mathrm{D}}_{S, T}(\mu)$. Write $\mathrm{D}$ for $\mathrm{D}_{\mathbb{I}, \mathbb{I}}$, where $\mathbb{I}$ consists of the non-dyadic reals in the interval $[0,1]$.

The disintegration operator on distributions having a continuous disintegration. Our main theorem is a characterization of the Weihrauch degree of D.

Proposition 4. EC $\leq_{\mathrm{sW}} \mathrm{D}_{\mathbb{N}, \mathbb{I}}$.

We show this by uniformly computing, given a representation $\beta_{x}$ of a function $x \in C(\mathbb{N}, \mathbb{S})$, a measure $\mu_{\beta_{x}} \in M_{1}(\mathbb{N} \times \mathbb{I})$ with the following properties.

Corollary 5. For every $X \in 2^{\omega}$ there is a continuous function $x \in C(\mathbb{N}, \mathbb{S})$ and a representation $\beta_{x}$ of $x$ such that

- the representation $\beta_{x}$ is computable in $X$,

- the disintegration $\mathrm{D}\left(\mu_{\beta_{x}}\right): \mathbb{I} \rightarrow M_{1}(\mathbb{N})$ along the projection $\pi: \mathbb{N} \times \mathbb{I} \rightarrow \mathbb{I}$ is continuous, and 
- any representation of the function $\mathrm{D}\left(\mu_{\beta_{x}}\right)$ computes $X^{\prime}$, the Turing jump of $X$.

We note that the construction of $\mu_{\beta_{x}}$ is a relativization of the construction of the measure used in [AFR10] to show that the conditional distribution of a computable random variable given another need not be computable.

Proposition 6. For any two computable topological spaces $S$ and $T$, we have $\mathrm{D}_{S, T} \leq_{\mathrm{W}} \operatorname{Lim}$.

In fact, we prove the following statement as well.

Corollary 7. Let $\mu \in M_{1}(S \times T)$ be such that $\mathrm{D}(\mu)$ is continuous on some set $X \subseteq T$ such that $\mu(S \times X)=1$. If $\beta$ is a representation of $\mu$, then there is a representation of $\mathrm{D}(\mu)$ computable from the Turing jump of $\beta$.

Using Propositions 4 and 6, we obtain our main theorem.

Theorem 8. $\mathrm{D} \equiv_{\mathrm{sW}} \mathrm{Lim}$.

The disintegration of specific distributions. Above we considered the disintegration operator on the space of measures for which the disintegration operator is continuous. Even for other measures, we can ask about the Weihrauch degree of the disintegration of the measure. As we saw in Corollaries 5 and 7 , in the case that disintegration is continuous, the disintegration is computable from the Turing jump of (a representation of) the distribution and bounded by the jump of (a representation of) the distribution. We finally consider the Weihrauch degree of disintegrations in the case when they are not continuous, and show that a single one can be as bad as Lim, even when it is defined everywhere; in fact our example is even absolutely continuous with respect to Lebesgue.

Proposition 9. There is a computable distribution $\mu \in M_{1}\left(\mathbb{N} \times 3^{\omega}\right)$ such that its conditional distribution $\mathrm{D}(\mu)$ (along $\pi: \mathbb{N} \times 3^{\omega} \rightarrow 3^{\omega}$ ) exists everywhere and satisfies $\mathrm{EC} \leq_{\mathrm{sW}} \mathrm{D}(\mu)$.

Proposition 10. Suppose $\mu \in M_{1}(S \times T)$ is a computable measure such that $\mathrm{D}_{S, T}(\mu)$ is total. Then $\mathrm{D}_{S, T}(\mu) \leq_{\mathrm{sW}} \operatorname{Lim}$.

\section{REFERENCES}

[AFR10] N. L. Ackerman, C. E. Freer, and D. M. Roy, On the computability of conditional probability, Preprint, arXiv:1005.3014, 2010.

[AFR11] _ Noncomputable conditional distributions, Proc. of the 26th Ann. IEEE Symp. on Logic in Comput. Sci. (LICS 2011), IEEE Computer Society, 2011, pp. 107-116.

[BG11] V. Brattka and G. Gherardi, Weihrauch degrees, omniscience principles and weak computability, J. Symbolic Logic 76 (2011), no. 1, 143-176.

[BHG13] V. Brattka, R. Hölzl, and G. Gherardi, Probabilistic Computability and Choice, Preprint, arXiv:1312.7305, 2013.

[Bra05] V. Brattka, Effective Borel measurability and reducibility of functions, Math. Log. Q. 51 (2005), no. $1,19-44$.

[FMNP95] D. A. S. Fraser, P. McDunnough, A. Naderi, and A. Plante, On the definition of probability densities and sufficiency of the likelihood map, Probab. Math. Statist. 15 (1995), 301-310.

[HR11] M. Hoyrup and C. Rojas, Absolute continuity of measures and preservation of randomness, Preprint, http://www.loria.fr/ hoyrup/abscont.pdf, 2011.

[HRW12] M. Hoyrup, C. Rojas, and K. Weihrauch, Computability of the Radon-Nikodym derivative, Computability 1 (2012), no. 1, 3-13.

[Kal02] O. Kallenberg, Foundations of modern probability, 2nd ed., Springer, New York, 2002.

[Pau12] A. Pauly, A new introduction to the theory of represented spaces, Preprint, arXiv:1204.3763, 2012.

[Pfa79] J. Pfanzagl, Conditional distributions as derivatives, Ann. Probab. 7 (1979), no. 6, 1046-1050.

[Tju75] T. Tjur, A constructive definition of conditional distributions, Preprint 13, Institute of Mathematical Statistics, University of Copenhagen, Copenhagen, 1975.

[Tju80] _ _ Probability based on Radon measures, Wiley Series in Probability and Mathematical Statistics, John Wiley \& Sons Ltd., Chichester, 1980.

[Zab79] S. Zabell, Continuous versions of regular conditional distributions, The Annals of Probability 7 (1979), no. 1, pp. 159-165. 


\section{The Weihrauch Lattice is too Small}

\author{
Andrej Bauer \\ University of Ljubljana \\ Andrej . Bauer@andrej . com
}

\author{
Kazuto Yoshimura \\ JAIST \\ k.yoshimura@jaist.ac.jp
}

July 3, 2014

In constructive reverse mathematics we care about implications of the form

$$
(\forall y \in B \cdot \psi(y)) \Longrightarrow(\forall x \in A . \phi(x)),
$$

where $\phi$ and $\psi$ are two principles that cannot be shown constructively valid. There is a natural way of proving such statements: given an arbitrary $x \in A$, find a suitable (not necessarily unique) $y \in B$ such that $\psi(y)$ implies $\phi(x)$. A cursory glance at the literature reveals numerous applications of the proof method, which therefore deserves a name. Recall that a relation $K \subseteq A \times B$ is total when $\forall x \in A . \exists y \in B . K(x, y)$. We write $\phi \subseteq A$ to indicate that $\phi$ is a predicate on $A$.

Definition 1. Suppose $\phi \subseteq A$ is a predicate on $A$ and $\psi \subseteq B$ is a predicate on $B$. Say that $\phi$ is instance reducible to $\psi$, written as $(\phi, A) \leq_{\mathrm{I}}(\psi, B)$ or just $\phi \leq_{\mathrm{I}} \psi$, if there is a total relation $K \subseteq A \times B$ such that

$$
\forall x \in A .(\exists y \in B . K(x, y) \wedge \psi(y)) \Rightarrow \phi(x) .
$$

The relation $K$ explains what it means for $x$ to be "suitable" for $y$. We say that $K$ is a witness for the reducibility. Often $K$ is the graph of a function, i.e., $K(x, y)$ is just $y=k(x)$ for some $k: A \rightarrow B$. Notice that (2) is equivalent to $\forall x \in A, y \in B . K(x, y) \wedge \psi(y) \Rightarrow \phi(x)$.

Theorem 2. Instance reducibilities form a bounded distributive lattice.

We must be careful about the interpretation of the above theorem. The lattice in question is large in the sense that its carrier consists of all sets and predicates. Also, $\leq_{\mathrm{I}}$ is a preorder rather than a partial order, so we should consider the structure up equivalence $\equiv_{\mathrm{I}}$, which is of course defined as mutual reducibility.

We say that a set $I$ is projective if it satisfies the axiom of choice: any family $\left(A_{i}\right)_{i \in I}$ of inhabited sets has a choice function, which is an element of the product $\prod_{i \in I} A_{i}$. For example, projectivity of $\mathbb{N}$ is precisely countable choice. Aczel's presentation axiom states that every set (or object) is covered by a projective one. It is typically valid in realizability models.

Theorem 3. If Aczel's presentation axiom holds then instance reducibilities form a complete lattice in which finite meets distribute over all joins. Furthermore, every predicate is equivalent to one on a projective set.

More precisely, the theorem states that set-indexed suprema and infima exist. Thus we do not have a large frame, as that would require arbitrarily large suprema.

The structure of instance reducibilities is very rich. For example, every truth value corresponds to a subset of the singleton set, which yields an anti-monotone embedding of truth values into instance reducibilities. For any $\phi \subseteq A$ and $B$ we may define its parameterization $\phi^{B} \subseteq A^{B}$ by $\phi^{B}(f) \Longleftrightarrow \forall y \in B . \phi(f(y))$. A reduction $\phi \leq_{\mathrm{I}} \psi^{B}$ then expresses the fact that we need $B$-many instances of $\psi$ to prove one instance of $\phi$.

The relevance of instance reducibilities is further bolstered by their relationship to Weihrauch reducibility. In the relative realizability model over Baire space, in which object-level realizers 
are elements of Baire space $\mathbb{N}^{\mathbb{N}}$ and morphisms are tracked by elements of the effective Baire space $\left(\mathbb{N}^{\mathbb{N}}\right)_{\text {eff }}$, we obtain the following correspondence. Recall that $\phi \subseteq A$ is $\neg \neg$-dense when $\neg \neg \phi(x) \Rightarrow \phi(x)$ for all $x \in A$.

Theorem 4. Instance reducibilities on $\neg \neg$-dense predicates correspond to Weihrauch degrees (with a top degree) in the relative realizability model over Baire space.

The correspondence immediately gives rise to fruitful interaction between constructive and computable mathematics. Realizers for constructive proofs of implication (2) give reductions between corresponding Weihrauch degrees. Insights gained by the study of Weihrauch degrees can be used to conclude lack of constructive implications between non-constructive principles (although such results require additional careful meta-mathematical analysis). The embedding of Medvedev degrees into Weihrauch lattice corresponds to the embedding of truth values into instance reducibilities, and so on.

We could check that Theorem 2 and half of Theorem 3 restrict to $\neg \neg$-dense predicates to obtain the usual distributive lattice structure on Weihrauch degrees, with an effective version of arbitrary infima. But we can do better by generalizing the notion of Weihrauch reducibility so that it matches perfectly instance reducibility.

Definition 5. An extended Weihrauch degree is a pair $(U, F)$ where $U \subseteq \mathbb{N}^{\mathbb{N}}$ and $F \subseteq U \times \mathbb{N}^{\mathbb{N}}$. A Weihrauch reduction $(U, F) \leq_{\mathrm{W}}(V, G)$ between two such degrees is given by a pair of partial computable maps $k: \mathbb{N}^{\mathbb{N}} \rightarrow \mathbb{N}^{\mathbb{N}}$ and $\ell: \mathbb{N}^{\mathbb{N}} \times \mathbb{N}^{\mathbb{N}} \rightarrow \mathbb{N}^{\mathbb{N}}$ such that:

1. for every $\alpha \in U, k(\alpha)$ is defined and $k(\alpha) \in V$,

2. for every $\alpha \in U$ and $\beta \in \mathbb{N}^{\mathbb{N}}$, if $(k(\alpha), \beta) \in G$ then $\ell(\alpha, \beta)$ is defined and $(\alpha, \ell(\alpha, \beta)) \in F$.

If Weihrauch degrees are viewed as multivalued maps between represented spaces, then the extended degrees correspond to partial multivalued maps. Natural examples of extended Weihrauch degrees which are not already Weihrauch degrees are readily available. Let us only mention the degree $\left(\mathbb{N}^{\mathbb{N}}, C\right)$ of formal Church's thesis, where $(\alpha, \beta) \in C$ if, and only if, $\beta(0)$ is the code of a Turing machine computing $\alpha$. Also known Weihrauch degrees may behave more naturally in their extended version. For instance, closed choice can be defined as an extended degree so that the empty set is included in the definition. Of course, it is impossible to choose anything from the empty set, but one might face a situation in which a reduction to closed choice must be performed without the implicit knowledge that the closed set in question is non-empty.

Again, it can be argued that extended Weihrauch degrees are of independent interest for computable mathematics. Moreover, they correspond precisely to instance reducibilities.

Theorem 6. Instance reducibilities correspond to extended Weihrauch degrees in the relative realizability model over Baire space.

Theorems 2 and 3 now apply to the extended Weihrauch degrees directly to give them the structure of a bounded distributive lattice with effective infima and suprema, indexed by arbitrary subsets of $\mathbb{N}^{\mathbb{N}}$.

The Weihrauch lattice embeds into its extended version: to every Weihrauch degree $F \subseteq$ $\mathbb{N}^{\mathbb{N}} \times \mathbb{N}^{\mathbb{N}}$ we assign an extended degree $\left(\operatorname{supp}_{F}, F\right)$ where $\operatorname{supp}_{F}=\{\alpha \mid \exists \beta .(\alpha, \beta) \in F\}$. Conversely, every extended Weihrauch degree $(U, F)$ may be restricted to the Weihrauch degree $F$. The restriction yields a monotone map which however does not preserve the lattice structure. The extension is in fact proper.

Instance reducibilities pave a way for generalizations of Weihrauch lattice to other models of computation. By interpreting Definition 1 in other models of computability, or even in any topos, we obtain a notion of Weihrauch-style reductions in that model. A preliminary investigation shows that in the effective topos we obtain a variant of truth-table reducibility. 


\title{
PROBABILISTIC COMPUTABILITY AND CHOICE
}

\author{
VASCO BRATTKA, GUIDO GHERARDI, AND RUPERT HÖLZL
}

Preliminary version of full paper available on: http://arxiv.org/abs/1312.7305

While the power of randomized algorithms has been studied in the discrete setting for a long time (see for instance [4]), very little is known for computations on real numbers. The aim of this talk is to give an overview of the systematic investigation we have recently carried out onto this largely unexplored field.

Randomization has no impact on what can be computed in principle as far as functions over natural numbers are concerned. In contrast, we will show that in the setting where inputs and outputs are infinite sequences, randomization can actually increase the computational power. In particular, a first very natural and significant scenario is the one where we allow a Turing machine to access, beside the information contained in the input, the auxiliary random advice information encoded by a sequence of coin tosses (that is, an element of Cantor space $2^{\mathbb{N}}$ ), and we assume that the success probability $\varepsilon$ that such random advice is useful to solve the problem is positive (i.e., $\varepsilon \in I=(0,1])$. Moreover we require the following failure recognition mechanism: the machine either produces a correct result or recognizes at some finite stage that the advice is unsuccessful and stops the computation. We will speak of Las Vegas computability to denote this type of computational concept ${ }^{1}$, and of Las Vegas computability over $R$ with measure $I \subseteq \mathbb{R}$ for the general case in which the advice information space $R$ and the measure interval $I$ may be differently chosen.

In our study, we have been able to show that the class of Las Vegas computable functions is closed under composition, which makes it a natural computational class, and we have found alternative characterizations of it. A first one is given by the probabilistic choice operator $\mathrm{P}_{I} \mathrm{C}_{Z}$, i.e., the restriction of the closed choice operator as studied in [2] to closed subsets $A$ of a represented Borel measurable space $Z$ with measure $\mu(A) \in I$ :

Theorem 1. Let $X$ and $Y$ be represented spaces, let $R \subseteq \mathbb{N}^{\mathbb{N}}$ be endowed with a Borel measure, let $I$ be an interval and let $f: \subseteq X \rightrightarrows Y$ be a multi-valued function. Then $f \leq{ }_{\mathrm{W}} \mathrm{P}_{I} \mathrm{C}_{R}$ iff $f$ is Las Vegas computable over $R$ with measure in $I$.

We abbreviate the notation for intervals, for instance, by writing " $>\varepsilon$ " instead of $(\varepsilon, \infty]$ and denote the positive choice operator $\mathrm{P}_{>0} \mathrm{C}_{Z}$ for $Z$ by " $\mathrm{PC}_{Z}$ " (as in [3]). As a corollary to Theorem 1 we deduce that $f$ is Las Vegas computable iff $f \leq{ }_{\mathrm{W}} \mathrm{PC}_{2^{\mathbb{N}}} \equiv_{\mathrm{W}} \mathrm{PC}_{[0,1]}$. We also obtain:

Theorem 2 (Probability dependency). Let $\varepsilon, \delta \in[0,1]$ and $R=2^{\mathbb{N}}$ or $R=[0,1]$. Then $\mathrm{P}_{>\varepsilon} \mathrm{C}_{R} \leq{ }_{\mathrm{W}} \mathrm{P}_{>\delta} \mathrm{C}_{R} \Longleftrightarrow \varepsilon \geq \delta$.

This theorem shows that the technique of probability amplification, which is well-known from the theory of randomized algorithms [4] over finite objects, fails for Las Vegas computability over infinite sequences.

A second characterization can be formulated in terms of the Weak Weak König's Lemma operator WWKL, which is the restriction of the Weak König's Lemma operator WKL as studied in [2] to trees $p \in 2^{\mathbb{N}}$ of positive measures:

Corollary 3. $f \leq_{\mathrm{W}}$ WWKL iff $f$ is Las Vegas computable.

Since WWKL $<_{W}$ WKL holds, Las Vegas computable functions constitute a proper subclass of non-deterministically computable functions (i.e., functions $f$ satisfying $f \leq_{W} W K L$ ).

\footnotetext{
${ }^{1}$ Our understanding of Las Vegas algorithms for infinite computations is very close to Babai's original understanding of this concept, see [1].
} 
VASCO BRATTKA, GUIDO GHERARDI, AND RUPERT HÖLZL

But in contrast to WKL, WWKL is not a cylinder (it does not compute the identity on the Baire space). This means that the Las Vegas computable functions do not coincide with the functions that are strongly Weihrauch reducible to WWKL.

Do mathematically meaningful examples of Las Vegas solvable problems that are not ordinarily computable exist? We will answer this question in the affirmative by some results proved by A. Pauly [5]:

Theorem 4. The task of finding matrix Nash equilibria is a Las Vegas computable problem that is not ordinarily computable.

The Weihrauch lattice actually provides in general a finer classification than that given by reverse mathematics and we also gain further evidence for this phenomenon in our new context: there are statements equivalent over $\mathrm{RCA}_{0}$ to $\mathrm{WWKL}_{0}$ that correspond to operators of different computational complexity. For instance, as we have seen, $\mathrm{PC}_{[0,1]}$ determines via $\leq_{\mathrm{W}}$ the class of Las Vegas computable functions, whereas $\mathrm{PC}_{\mathbb{R}}$ determines exactly the larger class of Las Vegas computable functions with finitely mind changes (i.e., compositions of a Las Vegas computable function and a function computable with finitely mind changes in either order).

A second natural scenario for infinite randomized computation can be obtained by rejecting the condition about the failure recognition in the following way:

Definition 5 (Probabilistic functions). Let $\left(X, \delta_{X}\right),\left(Y, \delta_{Y}\right)$ be represented spaces. A multivalued function $f: \subseteq X \rightrightarrows Y$ is called probabilistic, if there is a computable function $F: \subseteq \mathbb{N}^{\mathbb{N}} \times 2^{\mathbb{N}} \rightarrow \mathbb{N}^{\mathbb{N}}$ and a family $\left(A_{p}\right)_{p \in D}$ of measurable sets $A_{p} \subseteq 2^{\mathbb{N}}$ with $D:=\operatorname{dom}\left(f \delta_{X}\right)$ such that $(i) \mu\left(A_{p}\right)>0$ for all $p \in D$ and $(i i) \delta_{Y} F(p, r) \in f \delta_{X}(p)$ for all $p \in D$ and $r \in A_{p}$.

We have proved that this class is actually larger than that of Las Vegas computable functions, where a fundamental theorem of real analysis can serve as witness for the separation:

Theorem 6. The problem IVT of finding zeros of continuous functions changing their signs over the unit interval is probabilistically computable but not even Las Vegas computable with finitely many mind changes (it holds in fact IVT $\left.\right|_{\mathrm{W}} \mathrm{PC}_{\mathbb{R}}$ ).

\section{REFERENCES}

[1] László Babai. Monte-Carlo algorithms in graph isomorphism testing. Technical Report No. 79-10, Université de Montréal, Département de Mathématique et de Statistique, 1979.

[2] Vasco Brattka and Guido Gherardi. Weihrauch degrees, omniscience principles and weak computability. The Journal of Symbolic Logic, 76(1):143-176, 2011.

[3] Vasco Brattka and Arno Pauly. Computation with advice. In Xizhong Zheng and Ning Zhong, editors, CCA 2010, Proceedings of the Seventh International Conference on Computability and Complexity in Analysis, Electronic Proceedings in Theoretical Computer Science, pages 41-55, 2010.

[4] Rajeev Motwani and Prabhakar Raghavan. Randomized algorithms. Cambridge University Press, Cambridge, 1995.

[5] Arno Pauly. How incomputable is finding Nash equilibria? Journal of Universal Computer Science, 16(18):2686-2710, 2010.

Faculty of Computer Science, Universität der Bundeswehr München, Germany and Department of Mathematics \& Applied Mathematics, University of Cape Town, South Africa 2

E-mail address: Vasco.Brattka@cca-net.de

Faculty of Computer Science, Universität der Bundeswehr München, Germany

E-mail address: Guido.Gherardi@unibw.de

Faculty of Science, National University of Singapore, Singapore ${ }^{3}$

E-mail address: r@hoelzl.fr

\footnotetext{
${ }^{2}$ Vasco Brattka is supported by the National Research Foundation of South Africa.

${ }^{3}$ Rupert Hölzl was supported by a Feodor Lynen postdoctoral research fellowship of the Alexander von Humboldt Foundation and is supported by the Ministry of Education of Singapore through grant R146-000184-112 (MOE2013-T2-1-062).
} 


\title{
Exact real arithmetic and ODE systems with polynomial right hand sides *
}

\author{
F. Brauße ${ }^{1}$, M. Korovina ${ }^{2}$, N. Müller ${ }^{1}$, and A. van Ackeren ${ }^{3}$ \\ 1 Abteilung Informatikwissenschaften, Universität Trier, Germany \\ 2 A.P. Ershov Institute of Informatics Systems, Novosibirsk, Russia \\ 3 Mathematisches Institut, Universität Bonn, Germany
}

Ordinary differential equations (ODEs) have been addressed numerous times in computable analysis, consider for example [BHW08] or [Wei00] for general questions of computability. Major results in [Ko83] and [KC10] show that solving differential equations is closely related to the problem ' $\mathrm{P}=\mathrm{PSPACE}$ ' from discrete complexity theory. For special ODEs, on the other side, polytime computable right hand sides of the ODE lead to solutions that are computable in polynomial time as well. An analysis of the resulting complexity for onedimensional solutions with special initials value can already be found in [MM93]: If the right hand side of a one-dimensional ODE is holomorphic and computable in polynomial time, then the solution is polynomial time computable as well for all polytime computable initial values. The basic idea of the proof goes back to the well-known power series method for the solution of ODEs, where the differential equations lead to a recurrence relation for the coefficients of the ODE solution, see for example [BS85] or [Tes12]. It is worth noting that such classical mathematic textbooks do not consider aspects of computability or complexity.

In [MK10] the power series method had been analyzed for its applicability in computable analysis also in higher dimensions and for more general initial values than in [MM93]. Also a prototypical implementation working for linear differential equations had been described in brief. However, such implementations of ODE solvers in exact real arithmetic, thus being able to produce results of arbitrary precision, seem to be very rare. The authors only know about one further prototypical implementation mentioned in [EP07], which from its description seems to be based on the explicit construction of the solution using piecewise linear functions. This necessarily leads to a complexity that is exponential in the precision of the solution, so that approach can hardly be useful in practice without significant modifications.

In this paper we generalize the algorithm proposed in [MK10] to explicit ODEs with right hand sides that are polynomials. In addition to a discussion of the theory behind our approach we present a prototypical implementation in the iRRAM software package [Mül01,Mül09]. It is applicable to $d$-dimensional ODEs given by $d$ polynomials $F_{\nu}: \mathbb{R} \times \mathbb{R}^{d} \rightarrow \mathbb{R}(1 \leq \nu \leq d)$ defining the right hand sides of the ODE

$$
\dot{y}_{\nu}(t)=F_{\nu}\left(t, y_{1}(t), \ldots y_{d}(t)\right) \quad(1 \leq \nu \leq d) .
$$

\footnotetext{
* This research was partially supported by EU project PIRSES-GA-2011-294962, and RFBR grant N 11-01-00236-a.
} 
and vectors $\bar{w}_{0}$ defining initial values for the solution at a time $t_{0}$.

The implementation is able to compute

(a) a mapping solve : $\left(F_{\nu}, \bar{w}_{0}, t_{0}\right) \mapsto\left(a_{\nu, n}\right)$ for the power series $\sum a_{\nu, n} \cdot\left(t-t_{0}\right)^{n}$ of the unique solutions $y_{\nu}(t)$ near $t_{0}$,

(b) a mapping $R M:\left(F_{\nu}, \bar{w}_{0}, t_{0}\right) \mapsto(R, M)$ for lower bounds $R$ of the radii of convergence of these power series as well as bounds $M$ suitable for their evaluation, and

(c) a mapping sum : $\left(\left(a_{\nu, n}\right), R, M, t_{0}, t\right) \mapsto y_{n}(t)$ evaluating the solution at time $t$ for $\left|t-t_{0}\right|<R$.

For polynomials $F_{\nu}$, (a) turns out to be a simple algebraic computation, thus suited perfectly for an implementation in exact real arithmetic, also (b) can be implemented this way, (c) is already known from [MK10].

Using (c) to produce new initial values, iterations of the parts (a), (b), (c) allow to follow the solution on a longer interval. Here (b) plays the role of the adaptive step size known from ordinary ODE solvers.

We discuss aspects of computational complexity, especially the influence of the dimension $d$ and further characteristics of the ODE on the number of basic arithmetic steps in (a). Additionally we discuss how (b) can be improved. Finally we present a few benchmarks for well-known ODEs like the Van der Pol oscillator.

\section{References}

[BHW08] Vasco Brattka, Peter Hertling, and Klaus Weihrauch. A tutorial on computable analysis. In S. Barry Cooper, Benedikt Löwe, and Andrea Sorbi, editors, New Computational Paradigms: Changing Conceptions of What is Computable, pages 425-491. Springer, New York, 2008.

[BS85] I.N. Bronstein and K.A. Semendjajew. Taschenbuch der Mathematik. Verlag Nauka, Moskau, BSB B. G. Teubner Verlags-Gesellschaft, Leipzig, 1985.

[EP07] A. Edalat and D. Pattinson. A domain-theoretic account of Picard's theorem. LMS Journal of Computation and Mathematics, 10:83-118, 2007.

[KC10] Akitoshi Kawamura and Stephen A. Cook. Complexity theory for operators in analysis. In $S T O C$, pages 495-502, 2010.

[Ko83] Ker-I Ko. On the computational complexity of ordinary differential equations. Inf. Control, 58(1-3):157-194, 1983. .

[MK10] Norbert Müller and Margarita Korovina. Making big steps in trajectories. EPTCD, 2010.

[MM93] N. Th. Müller and B. Moiske. Solving initial value problems in polynomial time. In Proc. 22 JAIIO - PANEL '93, Part 2, pages 283-293, 1993. Buenos Aires, 1993.

[Mül01] Norbert Th. Müller. The iRRAM: Exact Arithmetic in C++. Lecture notes in computer science, 2991:222-252, 2001.

[Mü109] Norbert Th. Müller. Enhancing imperative exact real arithmetic with functions and logic. Technical report, software presentation at the CCA 2009 conference, Ljubljana, 2009.

[Tes12] G. Teschl. Ordinary Differential Equations and Dynamical Systems. Graduate studies in mathematics. American Mathematical Soc., 2012.

[Wei00] Klaus Weihrauch. Computable analysis: an introduction. Springer-Verlag New York, Inc., Secaucus, NJ, USA, 2000. 


\title{
Computational aspects of simultaneous Diophantine approximation
}

\author{
Keith Briggs \\ BT Technology, Services and Operations \\ Adastral Park, Martlesham Heath, UK \\ keith . briggs@bt.com \\ CCA2014, Darmstadt, 2014-07-21 to 24
}

Last modified 2014-06-04 10:28

\section{Abstract}

The classical one-dimensional Diophantine approximation concerns the finding of rational numbers close to a given irrational number. This problem is fully solved by the classical theory of continued fractions, but from the computational point of view, this theory already hides some difficulties. How is the input irrational number to be specified - by rational bounds, a sequence of rational approximations, or something else? If it uses floating-point arithmetic, a fully rigorous continued fraction algorithm will need some kind of error analysis and precision control. I will mention some approaches to this problem, but my main emphasis will be two-dimensional generalizations of such algorithms.

The two-dimensional problem of approximation of a pair of irrationals by a pair of rationals with the same denominator is much harder, and for the computation of so-called best approximants, no classical algorithm exists. But little-known algorithms by Vaughan Clarkson ( $\mathrm{PhD}$ thesis, University of Queensland 1997) do exist, and I discuss the computational issues in implementing these.

I will thus present some results from the first fully-rigorous implementation of an algorithm for finding the sequence of best approximants for the simultaneous rational approximation of two irrationals; this allows investigation of some unsolved problems in elementary number theory. The ultimate target is in fact the study of some of these unsolved problems, such as which pair of irrationals is the worst approximable (in the sense of the golden mean in one dimension).

\section{The continued fraction algorithm}

The problem is: given $x \in \mathbb{R}^{+} \backslash \mathbb{Q}$ and an integer $n$, find $n$ terms of the sequence of best approximation denominators, defined as the unique infinite sequence $Q=\left\{q_{i}\right\}\left(i=0,1,2, \ldots ; q_{0}=\lfloor x\rfloor\right)$ for which $\left|q_{i} x\right|<\left|q_{j} x\right| \forall j<i$ (here $\cap$ is defined as the distance to the nearest integer). Example: $x=(\sqrt{5}-1) / 2, Q=\{1,1,2,3,5,8,13,21,34, \ldots\}$. This elementary algorithm forms a prototype for the higher-dimensional algorithms which we really want to study.

We are interested in practical computability, in this precise sense: if a nested sequence of rational bounds for $x$ can be computed, can we compute $Q$ (and if so, with what complexity)? The answer is yes, and one simple way is to apply Euclid's algorithm for rationals, with purely rational arithmetic, separately to the lower and upper bounds. A simple theorem tells us that as long as these two sequences agree, then they are correct for $x$. When they first disagree, we have to compute tighter rational bounds for $x$, apply a correction to account for the steps of Euclid's algorithm already made, and continue. This works, but the correction step is inelegant and the whole algorithm may not have optimal complexity. Euclid's algorithm is: $A=\left[\begin{array}{ll}0 & 1 \\ 1 & 0\end{array}\right]$; for $i=0,1,2, \ldots: a_{i} \leftarrow\lfloor x\rfloor, A \leftarrow\left[\begin{array}{ll}0 & 1 \\ 1 & a_{i}\end{array}\right] A, q_{i} \leftarrow A_{22}, x \leftarrow 1 /\left(x-a_{i}\right)$, endfor. 
However, I would like to exploit mpfr, the correctly-rounding floating-point library, as this will effectively do the bound calculations for me (by rounding down and up). If I build a ball arithmetic (center and radius) on top of mpfr, then I can remove the requirement to run Euclid's algorithm twice, but I still have to stop at the point where the floor cannot be determined because the radius has got too big, and the fix-up step is still required. So it is a fundamental question as to whether there is a better continued fraction algorithm which runs indefinitely. In any case, I will assume ball arithmetic for all subsequent algorithms.

\section{Two-dimensional algorithms}

We now move to two dimensions, where analogous algorithms are not generally known. My aim is to implement a practical algorithm for computing the sequence of best Diophantine approximations for two dual problems, given a pair of irrationals $\left(\alpha_{1}, \alpha_{2}\right)$.

- Firstly, simultaneous approximation: make the "radius" $f\left(\left|q \alpha_{1}\right|,\left|q \alpha_{2}\right|\right)$ small, where $f$ is some norm on $\mathbb{R}^{2}$, while not making the "height" $q$ not too big.

- Secondly, minimization of a linear form: that is, make the radius $\left|q+\alpha_{1} p_{1}+\alpha_{2} p_{2}\right|$ small, while not making the height $f\left(\alpha_{1}, \alpha_{2}\right)$ (measured by some norm $f$ on $\left.\mathbb{R}^{2}\right)$ too big.

For both problems we have a sequence $Q$ of best approximation denominators, analogous to the continued fraction case. The fundamental problem is:

- Given $\left(\alpha_{1}, \alpha_{2}\right)$, can we compute $Q$ (better than simply trying $\left.q=1,2,3, \ldots\right)$ ?

- Given $\left(\alpha_{1}, \alpha_{2}\right)$ in the form of nested rational intervals, can we compute $Q$ to arbitrary length?

The answer to the first question is yes; an algorithm was given by Clarkson (1997) and proved correct. My contribution here is an answer to the second question (yes, with one caveat).

However, we still have the practical software-design issues of handling the ball arithmetic (or something equivalent, such as interval arithmetic). Although there is not space for details here, it turns out that Clarkson's algorithm 6.1 can be expressed as a main loop which only handles integer 3-vectors (see below; all subscripted variables are integer 3-vectors), and a function $L$ which (and only which) handles the real arithmetic. Inside $L$ (which simply return true or false), some comparison are made, and this is where ball arithmetic is used. Clarkson groups the radius and height functions (mentioned above) into a 2-tuple $\rho h(x) \equiv(\rho(x), h(x))$ (similarly $h \rho$ ) and defines sorting of such objects lexicographically. $L$ is then defined by $L(x, y, z)=(\rho h(x)<\rho h(z) \wedge \rho h(y)<\rho h(z)) \wedge(h \rho(x)<h \rho(y)) \vee \neg[\rho h(x)<\rho h(z) \wedge \rho h(y)<\rho h(z)] \wedge \rho h(x)<\rho h(y)$. Strictly speaking, the sequence $Q$ is not computable, as in some cases of $\left(\alpha_{1}, \alpha_{2}\right)$, some of the comparisons may actually be of equal quantities, in which case the ball arithmetic will enter an infinite loop. Nevertheless, in many cases of practical interest, this does not happen and we may now investigate some unsolved number-theoretic problems with computer experiments, such as whether the values of the linear form in the example in the plot are bounded below by a strictly positive constant (and if so, how big is that constant?). 

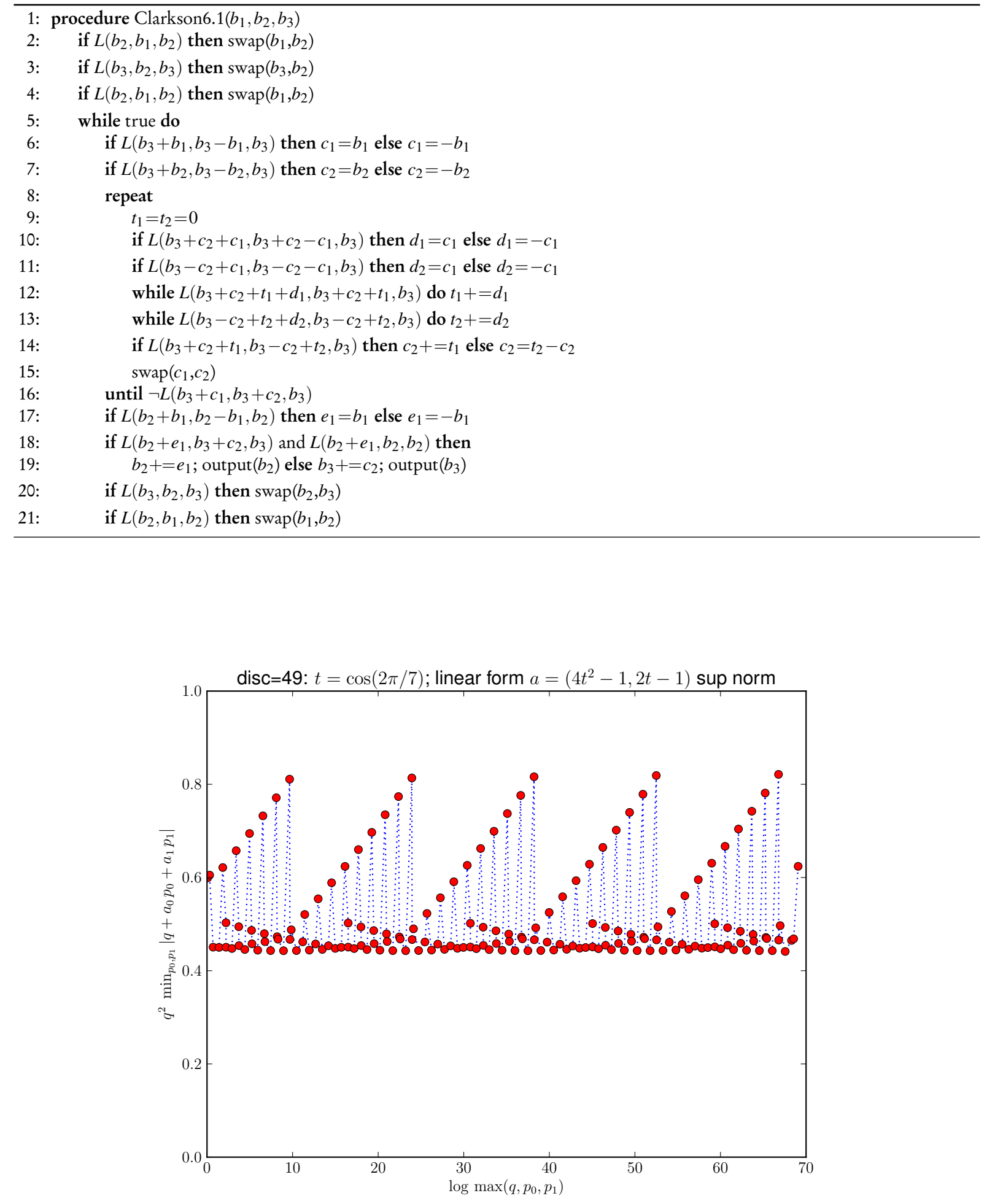


\title{
Computability of Solutions of Stochastic Differential Equations
}

\author{
Pieter Collins
}

7 April 2014

\begin{abstract}
In this talk, we consider computability of solutions of the stochastic differential equation

$$
d X(t)=f(X(t)) d t+g(X(t)) d W(t)
$$

where $f, g: \mathbb{R} \rightarrow \mathbb{R}$ are Lipschitz functions, $W$ is the Wiener process and $X$ is a real-valued stochastic process.

The main result is that given a fixed initial condition $X(0)=x_{0}$, the process $X$ is a computable random variable taking values in $C([0, T] ; \mathbb{R})$. The proof is a fairly straightforward effectivisation of classical existence and uniqueness results; see [Fri75, BP06, Eva13]. One first defines the Itō stochastic integral $\int_{0}^{T} X(t) d W(t)$ and then solves the integral version of $(\dagger)$. The main difficulty is is effectivising the limiting procedures involved.

The effectivisation depends crucially on the notion of random variable introduced in [Col12] based on an idea of [CS09]. The type of measurable random variables on a probability space $(\Omega, P)$ taking values in a Polish space $(E, d)$ is the effective completion of piecewise-continuous random variables under the Fan metric

$$
d(X, Y)=\sup \left\{\varepsilon \in \mathbb{Q}^{+} \mid P(\{\omega \in \Omega \mid d(X(\omega), Y(\omega))>\varepsilon\})>\varepsilon\right\} .
$$

While our approach shows that that sample paths are continuous almost everywhere, it does not allow for sample paths to be computed as a function of $\omega \in \Omega$, even though a computable version of Brownian motion exists [Col12].
\end{abstract}

\section{References}

[BP06] Stefano Bonaccorsi and Enrico Priola. From Brownian motion to stochastic differential equations. 10th Internet Seminar, 2006.

[Col12] Pieter Collins. Computable probability theory and stochastic processes. In Proceedings of the Ninth International Conference on Computability and Complexity in Analysis, 2012.

[CS09] Thierry Coquand and Bas Spitters. Integrals and valuations. J. Logic Analysis, 1(3):1-22, 2009.

[Eva13] Craig Evans. An Introduction to Stochastic Differential Equations. AMS, 2013.

[Fri75] Avner Friedman. Stochastic Differential Equations. Dover, 1975. 


\title{
Separating Variants of LEM, LPO, and MP
}

\author{
Matt Hendtlass and Robert Lubarsky
}

Omniscience principles are commonly used in constructive mathematics. The best known ones likely are:

- LPO: For any binary sequence $\alpha$, either $\alpha(n)=0$ for all $n$ or there exists $n$ such that $\alpha(n)=1$.

- WLPO: For any binary sequence $\alpha$, either $\alpha(n)=0$ for all $n$ or it is not the case that $\alpha(n)=0$ for all $n$.

- LLPO: For any binary sequence $\alpha$ with at most one non-zero term, either $\alpha(n)=0$ for all even $n$ or $\alpha(n)=0$ for all odd $n$.

Closely related to those is:

- WKL: Every infinite decidable tree has an infinite branch.

In [11] Richman defined a hierarchy of principles $\operatorname{LLPO}_{\nu}(\nu \in \omega+1, \nu \geqslant 2)$ related to LLPO:

- LLPO $\mathbf{L}_{\nu}$ : Let $\left(P_{i}\right)_{i<\nu}$ be a decidable partition of $\omega$ into blocks of size $\omega$, and let $\alpha$ be a binary sequence with at most one non-zero term. Then there exists $k<\nu$ such that $\alpha(m)=0$ for all $m \in P_{k}$.

The mother of all omniscience principles is:

- LEM: For any proposition $A$, either $A$ is true or $A$ is false,

Corresponding to (and implying) WLPO is:

- WLEM: For any proposition $A$, either $\neg A$ or $\neg \neg A$.

Similar to $\mathrm{LLPO}_{n}$ is a hierarchy of weakenings of WLEM:

- $\mathbf{W L E M}_{n}: \quad \neg \bigvee_{i, j<n, i \neq j} A_{i} \wedge A_{j} \longrightarrow \bigvee_{i<n} \neg A_{i}$

Another principle commonly considered in constructive reverse mathematics is:

- MP: If not all terms of $\alpha$ are zero, then, for some $n, \alpha(n)=1$.

There are two standard weakenings of MP: 
- WMP:

$\forall \alpha[\forall \beta(\neg \neg \exists n(\beta(n)=1) \vee \neg \neg \exists n(\alpha(n)=1 \wedge \beta(n)=0)) \rightarrow \exists n \alpha(n)=1]$.

- $\mathbf{M P}^{\vee}$ : If $\alpha$ has at most one non-zero term and it is impossible for all terms of $\alpha$ to be zero, then either all even terms or all odd terms are zero.

We shall also consider weakenings of $\mathrm{MP}_{n}^{\vee}$ and $\mathrm{MP}_{\omega}^{\vee}$ of $\mathrm{MP}^{\vee}$ corresponding to and implied by $\mathrm{LLPO}_{n}$ and $\mathrm{LLPO}_{\omega}$.

We show here that all of the implications among these principles that are not easily seen to be true are in fact not provable over IZF (usually with DC). Some of these were already known, many were not. In particular, we show:

- LPO does not imply $\mathrm{WLEM}_{\omega}$.

- WKL does not imply WLPO.

- WLEM does not imply WMP, and $\mathrm{WLEM}_{n+1}$ does not imply $\mathrm{MP}_{n}^{\vee}$.

- Even the weakest principle we consider, $\mathrm{MP}_{\omega}^{\vee}$, is not provable.

Our techniques are to provide Kripke models, topological models, and mixes of the two, as counter-examples. Even in those cases where the independence result is already known, there is a contrast in the methods, since many of extant proofs are realizability constructions, and sometimes are over weaker base theories.

\section{References}

[1] Y. Akama, S. Berardi, S. Hayashi and U. Kohlenbach, 'An arithmetical hierarchy of the law of excluded middle and related principles', in: Proc. of the 19th Annual IEEE Symposium on Logic in Computer Science (LICS '04), p. 192-201, IEEE Press, 2004.

[2] R-M. Chen and M. Rathjen, 'Lifschitz realizability for intuitionistic Zermelo-Fraenkel set theory', Arch. Math. Logic 51 (7-8), p. 789-818, 2012.

[3] R.J. Grayson, 'Heyting-valued semantics', Logic Colloquium '82, Studies in Logic and the Foundations of Mathematics 112, North-Holland, p. 181-208, 1984.

[4] H. Ishihara, 'Markovs principle, Churchs thesis and Lindelöfs theorem', Indag. Math. 4 (3), p. 321-325, 1993.

[5] U. Kohlenbach, 'Relative constructivity', Journal of Symbolic Logic 63, p. 1218-1238, 1998.

[6] U. Kohlenbach, 'On weak Markov's principle,' Math. Logic Quarterly 48, Supplement 1 p. 59-65, 2002. 


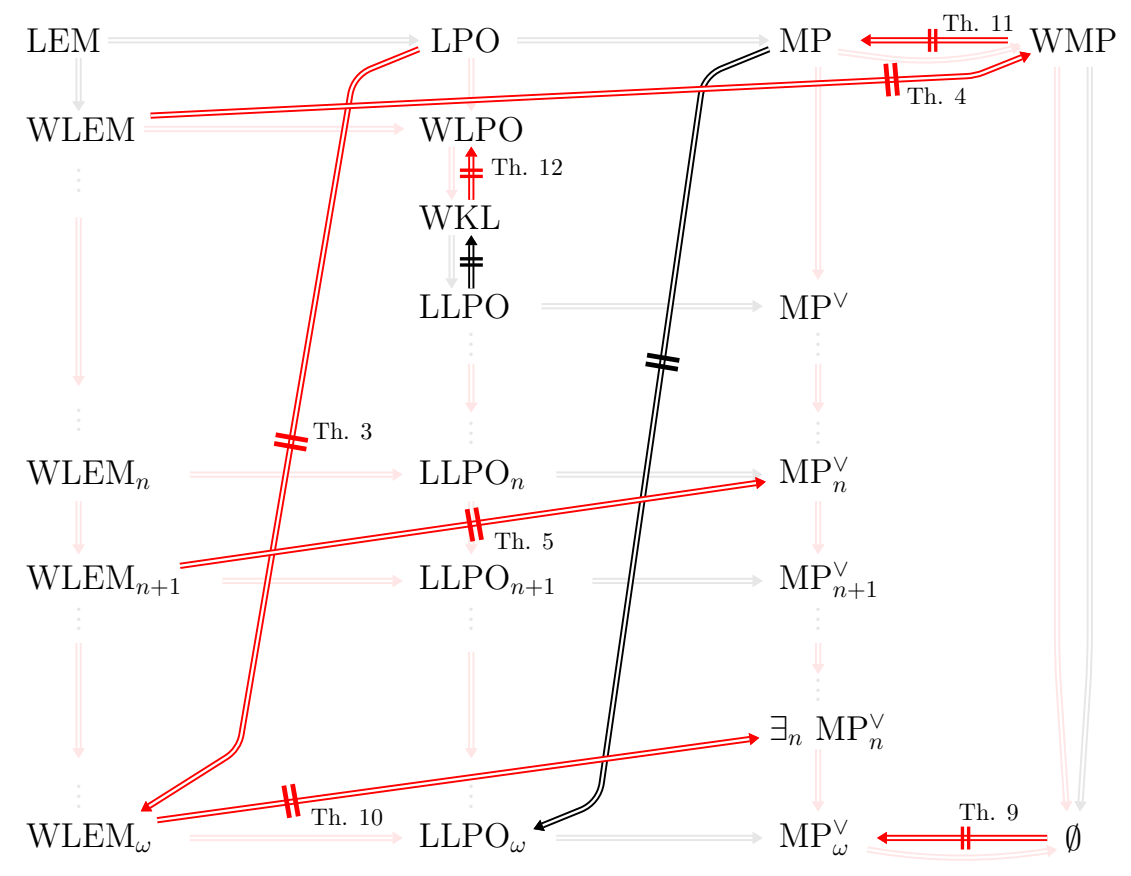

Figure 1: The non-derivabilities that give all the non-reversals between the fragments of LEM we study here.

[7] U. Kohlenbach, Applied Proof Theory: Proof Interpretations and their Use in Mathematics, Springer, 2008.

[8] M. Krol, 'A Topological model for intuitionistic analysis with Kripke's Scheme', Zeitschr. f. math. Logic und Grundlagen d. Math. 24, p. 427-436, 1978.

[9] M. Mandelkern, 'Constructively complete finite sets', Zeitschr. f. math. Logic und Grundlagen d. Math. 34, p. 97-103, 1988.

[10] J. Moschovakis, 'A Topological interpretation of second-order intuitionistic arithmetic', Compositio Mathematica 26, p. 261-276, 1973.

[11] F. Richman, 'Polynomials and linear transformations', Linear Algebra Appl. 131, p. 131-137, 1990.

[12] D. van Dalen, 'An interpretation of intuitionistic analysis', Annals of Mathematical Logic 13 (1), p. 1-43, 1978.

[13] R.E. Vesley, 'Realizing Brouwer's sequences', Annals of Pure and Applied Logic 81, p. 25-74, 1996. 


\title{
Computable transition points
}

\author{
Zvonko Iljazović and Bojan Pažek \\ University of Zagreb, Croatia
}

If $f:[0,1] \rightarrow \mathbb{R}$ is a computable function such that $f(0)<0$ and $f(1)>0$, then there exists a computable number $x \in[0,1]$ such that $f(x)=0$.

Let $S=\mathbb{R} \times\{0\}$. Let $f:[0,1] \rightarrow \mathbb{R}$ be a computable function and let $K$ be the graph of $f$, i.e. $K=\{(x, f(x)) \mid x \in[0,1]\}$. Then $f$ has a computable zero-point if and only $K$ intersects $S$ in a computable point.

Since $S$ and $K$ are computable sets in $\mathbb{R}^{2}$, the question which arises is the following: under what assumptions the intersection of two computable sets contains a computable point?

There exists a computable function $f:[0,1] \rightarrow \mathbb{R}$ which has zero-points, but none of them is computable. For such a function $f$ we have $K \cap S \neq \emptyset$, but $K$ does not intersect $S$ in a computable point. Note that in this case $K$ cannot intersects both of the sets $U=\left\{(x, y) \in \mathbb{R}^{2} \mid y<0\right\}$ and $V=\left\{(x, y) \in \mathbb{R}^{2} \mid y>\right.$ $0\}$.

However, if $f:[0,1] \rightarrow \mathbb{R}$ is computable and $f(0)<0$ and $f(1)>0$, then its graph $K$ intersects both $U$ and $V$. Since the graph of a continuous function is compact and connected (i.e. a continuum), the question is this: if $K$ is a computable continuum which intersects $U$ and $V$, does $K$ intersect $S$ in a computable point?

It turns out that the answer to this question is negative. However, the graphs of computable functions $[0,1] \rightarrow \mathbb{R}$ are not just any computable continua, they are computable arcs. So we wonder what can be said in the case when $K$ is a computable arc such that both $U$ and $V$ contain one of the endpoints of $K$. On the other hand, we can generalize the ambient space by taking any computable metric space $(X, d, \alpha)$ and we can take $S$ to be any subset of $X$ which separates $X$ into two computably enumerable open sets $U$ and $V$.

Moreover, we examine spaces more general than arcs, the spaces called chainable continua. A metric space $(X, d)$ is said to be continuum chainable from $a$ to $b, a, b \in X$, if $(X, d)$ is a continuum and for each $\varepsilon>0$ there exists a finite sequence of open sets $C_{0}, \ldots, C_{m}$ which cover $X$, whose diameters are less than $\varepsilon$, such that $a \in C_{0}, b \in C_{m}$ and such that

$$
C_{i} \cap C_{j}=\emptyset \text { if }|i-j|>1 .
$$

For example, if $K$ is an arc with endpoints $a$ and $b$, then $K$ is a continuum chainable from $a$ to $b$. On the other hand, $K=\{(0, y) \mid y \in[-1,1]\} \cup\{(x, \sin x) \mid$ $0<x \leq 1\}$ is a continuum chainable from $(0,-1)$ to $(1, \sin 1)$, but $K$ is not an arc. 
Suppose $(X, d, \alpha)$ is a computable metric space. Let $U$ and $V$ be disjoint and computably enumerable open sets and let $K$ be a computable compact set in this space. Let $S=X \backslash(U \cup V)$. Suppose $K$ is a continuum chainable from $a$ to $b$, where $a$ and $b$ are computable points, $a \in U, b \in V$.

We prove the following: if $K \cap S$ is totally disconnected, then $K \cap S$ contains a computable point.

This result implies the following: if $A$ is a computable arc with computable endpoints such that both $U$ and $V$ contain one of the endpoints of $A$, then $A$ intersects $S$ in a computable point.

\section{References}

[1] Vasco Brattka. Plottable real number functions and the computable graph theorem. SIAM J. Comput., 38(1):303-328, 2008.

[2] Vasco Brattka and Gero Presser. Computability on subsets of metric spaces. Theoretical Computer Science, 305:43-76, 2003.

[3] Charles O. Christenson and William L. Voxman. Aspects of Topology. Marcel Dekker, Inc., New York, 1977.

[4] Zvonko Iljazović. Chainable and Circularly Chainable Co-c.e. Sets in Computable Metric Spaces. Journal of Universal Computer Science, 15(6):12061235, 2009.

[5] Takayuki Kihara. Incomputability of Simply Connected Planar Continua Computability, 1(2):131-152, 2012.

[6] Joseph S. Miller. Effectiveness for Embedded Spheres and Balls. Electronic Notes in Theoretical Computer Science, 66:127-138, 2002.

[7] S.B. Nadler. Continuum theory. Marcel Dekker, Inc., New York, 1992.

[8] Marian Pour-El and Ian Richards. Computability in Analysis and Physics. Springer-Verlag, Berlin-Heielberg-New York, 1989.

[9] Ernst Specker. Der Satz vom Maximum in der rekursiven Analysis. Constructivity in Mathematics (A. Heyting, ed.). North Holland Publ. Comp., Amsterdam, 254-265, 1959.

[10] Klaus Weihrauch. Computable Analysis Springer, Berlin, 2000. 


\title{
Analytic Functions in iRRAM*
}

\author{
Akitoshi Kawamura $^{1}$, Florian Steinberg ${ }^{2}$, Holger Thies ${ }^{1,2}$ \\ 1 The University of Tokyo (JAPAN), ${ }^{2}$ TU Darmstadt (GERMANY)
}

The Type-2 Theory of Effectivity provides a sound framework for investigating the computability of real numbers, sequences, functions, and operators. It is well known that an analytic function on a compact domain is computable if and only if its sequence of Taylor coefficients is computable. However, an algorithm evaluating a given power series must in addition to said coefficient sequence be provided with additional information about its rate of convergence [Mü195]. On simple domains, this information can be realized by two integer parameters. Moreover, Real Complexity Theory shows that this representation renders the usual operations on analytic functions uniformly computable within parameterized polynomial time [KMRZ12]. This is in contrast with the situation for smooth functions where maximization has been shown to correspond to the $\mathcal{P}$ vs. $\mathcal{N} \mathcal{P}$ Millennium Prize Problem [KF82] and integration to the even stronger $\mathcal{F P}$ vs. \#P question [Fri84, Ko91]. Theory thus suggests a mixed real/integer data structure and interface declaration for practical and efficient implementations of power series in imperative programming. iRRAM constitutes a free library to build upon, conveniently providing an abstract data type REAL in $\mathrm{C}++$.

We present such a prototype implementation of power series and analytic functions on a fixed line segment. The data type encodes a germ enriched with integer information as described above; it supports basic operations like pointwise addition and multiplication, composition, differentiation and integration. Evaluation is realized by analytic continuation using iterated evaluation and interpolation. We proceed to explore the practical performance of our implemented and compare its empirical behaviour with the theoretical predictions.

Let $\mathbb{D}$ denote the closed complex unit disc. The analytic functions on $\mathbb{D}$ are in one to one correspondence with the sequences $\bar{a}=\left(a_{n}\right)_{n \in \mathbb{N}}$, whose radius of convergence is strictly larger than 1 via the assignment

$$
\bar{a} \mapsto f_{\bar{a}}, \quad \text { where } \quad f_{\bar{a}}(x)=\sum_{n \in \mathbb{N}} a_{n} x^{n},
$$

resp.

$$
f \mapsto\left(\frac{f^{(n)}(x)}{n !}\right)_{n \in \mathbb{N}}
$$

These assignments can easily be seen to be discontinuous and therefore not computable. Thus consider tuples $\left(A, k,\left(a_{n}\right)_{n \in \mathbb{N}}\right)$, where $A$ and $k$ are integers such that

- $r:=\sqrt[k]{2}$ is strictly smaller than the radius of convergence of $\left(a_{n}\right)_{n \in \mathbb{N}}$

- $\left|a_{n}\right| r^{n} \leq A$ for all $n \in \mathbb{N}$.

* Supported in part by JSPS Kakenhi projects 23700009 and 24106002, by 7th EU IRSES project 294962 , by $D F G$ project Zi $1009 / 4-1$, and by $D A A D$. 


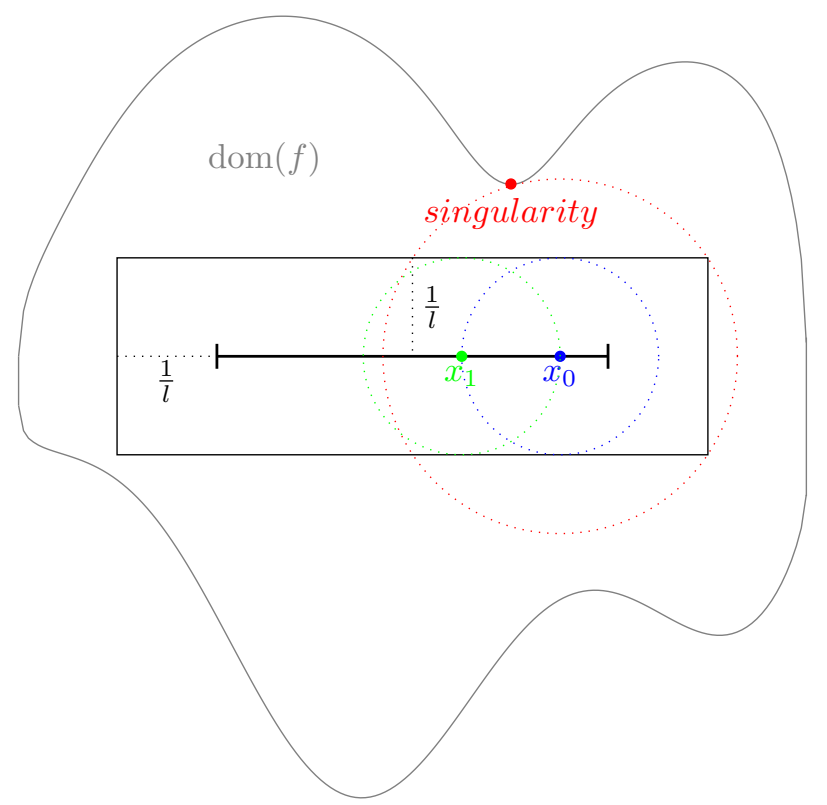

Figure 1: A function $f$ defined on an open superset of a closed rectangle with marked singularity. We consider a germ around $x_{0}$.

The evaluation operator $\left(A, k,\left(a_{n}\right)_{n \in \mathbb{N}}, x\right) \mapsto f_{\bar{a}}(x)$ becomes computable in time polynomial in the binary output precision, in $\log (A)$, and in $k$. Moreover, addition, integration, maximization and many other basic operations on power series can be shown to be parameterized polynomial time computable if considered as multivalued operators on such tuples.

The data type BASE_ANA realizes the above theoretical considerations as an interface class for iRRAM. There is an constructor, constructing a BASE_ANA object from a given power series together with integers $A$ and $k$, where the user has to make sure they fulfill the above relations. The standard operators,$+ *$ are overloaded to also work for these objects and differentiation as well as integration and evaluation are implemented.

Our implementation of a data type ANA_RECT for analytic functions on a line segment follows the ideas from [KMRZ12] closely. That is, a function $f$ on a line segment can either be represented by its Taylor sequence around some point $x_{0}$ on the line segment and integer parameters $A$ and $k$ such that $f^{(j)}(x) \leq A k^{j} j$ ! for all $x$ in the line segment, or by an evaluation procedure together with constants $B$ and $l$ such that $f$ allows an analytic continuation onto an rectangle as indicated in fig. 1 and $|f(x)| \leq B$ for all $x$ in that rectangle. Implementation of basic operations like addition, multiplication, differentiation and integration for these objects is straight forward.

The availability of the additional discrete information makes it possible to switch back and forth between the two representations. Note, however, that even with $A$ and $k$ available, the Taylor expansion of $f$ around $x_{0}$ can not directly be used to evaluate the function, as the series may diverge due to singularities being close (cmp. fig. 1). This is where analytic continuation becomes necessary: By evaluating the power series, we can compute the coefficients of the Taylor series around a different base point $x_{1}$ and by iterating this process, we can evaluate in any point on the line segment. This can also be understood as an instance of ANA_RECT alternately calling different constructors for shifted $a_{32}{ }_{3}$ caled BASE_ANA objects. 
To compute the Taylor series around some point of the domain when given a triple $(f, B, l)$ as above, we use the interpolation procedure described in [Mül87]: Computing the coefficient $a_{m}:=\frac{1}{m !} f^{(m)}\left(x_{1}\right)$ can be done by a interpolating $f$ with a polynomial $P$ and compute the derivatives $P^{(k)}\left(x_{1}\right)$. If $P$ is the unique polynomial interpolating $f$ at $j+1$ points for any $m \leq j$ the error made when computing $a_{m}$ through $P$ can be approximated by

$$
\left|a_{m}-\frac{1}{m !} P^{(k)}(0)\right| \leq B\left(\frac{1}{2 l}\right)^{j+1-m} 4^{\frac{4^{j+1}}{\left(\frac{1}{l}\right)^{j+1}}} .
$$

The interpolation is done by partitioning the domain into equidistant points $z_{0}, \ldots$, $z_{2 m+1}$ with distance $h$ and using the Lagrange Interpolation Formula

$$
P(z)=\sum_{i=0}^{2 m} f\left(z_{i}\right) L_{m, i}(z) \text { with } L_{m, i}=\prod_{\substack{0 \leq j \leq 2 m \\ j \neq i}} \frac{z-z_{j}}{z_{i}-z_{j}} .
$$

It can be shown that

$$
a_{m} \approx \sum_{i=0}^{2 m} f\left(z_{i}\right) \frac{h^{-m} l_{m, i}}{i !(2 m-i) !}
$$

for $l_{m, i}$ that can be computed in time polynomial in $m$. Therefore the same holds for the right hand side.

As a practical example, we try to continuously extend the square root function around the boundary of the unit square.

\section{References}

[Fri84] Harvey Friedman, The computational complexity of maximization and integration, Adv. in Math. 53 (1984), no. 1, 80-98. MR 748898 (86c:03037)

[KF82] Ker-I Ko and Harvey Friedman, Computational complexity of real functions, Theoret. Comput. Sci. 20 (1982), no. 3, 323-352. MR 666209 (83j:03103)

[KMRZ12] Akitoshi Kawamura, Norbert Th. Müller, Carsten Rösnick, and Martin Ziegler, Parameterized Uniform Complexity in Numerics: from Smooth to Analytic, from NP-hard to Polytime, arXiv preprint (2012).

[Ko91] Ker-I Ko, Complexity theory of real functions, Progress in Theoretical Computer Science, Birkhäuser Boston Inc., Boston, MA, 1991. MR 1137517 (93i:03057)

[Mül87] Norbert Th. Müller, Uniform computational complexity of Taylor series, Proc. 14th International Colloquium on Automata, Languages, and Programming, LNCS, vol. 267, Springer, 1987, pp. 435-444.

[Mü195] _ Constructive aspects of analytic functions, Proc. Workshop on Computability and Complexity in Analysis, InformatikBerichte, vol. 190, FernUniversität Hagen, 1995, pp. 105-114. 


\title{
On the Computational Power of Algorithmically Random Constants in Blum-Shub-Smale Machines
}

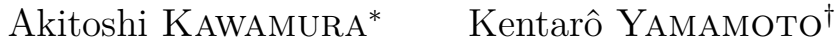

A Blum-Shub-Smale (BSS) machine over $\mathbb{R}$, which can operate on elements of $\mathbb{R}$ in a unit time, is one of the models for the theory of computation over real numbers. An interesting problem regarding BSS machines is to determine the computational power of BSS machines relative to other computational models.

Allender, Bürgisser, Kjeldgaard-Pedersen and Miltersen [1] related BSS machines with ordinary Turing machines and straight-line programs (SLPs), an algebraic model of computation perused in algebraic complexity theory. To see their work in detail, let us introduce a few notations. Let $L_{0}$ denote the discrete decision problem of deciding whether a given 0-variate SLP (that uses 0 and 1 as constants) represents a positive number. Also, for a finite set $S \subset \mathbb{R}$, let $\operatorname{BP}\left(\mathbf{P}_{\mathbb{R}}^{S}\right)$ denote the class of discrete decision problems solved by polynomialtime BSS machines that may use only elements of $S$ as machine constants, i. e., the real numbers that the machine may access when performing operations on $\mathbb{R}$. The union of $\operatorname{BP}\left(\mathbf{P}_{\mathbb{R}}^{S}\right)$ over all finite sets $S \subset \mathbb{R}$ is denoted $\operatorname{BP}\left(\mathbf{P}_{\mathbb{R}}\right)$. It is easily seen that $\mathbf{P}^{L_{0}} /$ poly $\subset \operatorname{BP}\left(\mathbf{P}_{\mathbb{R}}\right)$; Allender et al. conjectured that, in fact, $\mathbf{P}^{L_{0}} /$ poly $=\operatorname{BP}\left(\mathbf{P}_{\mathbb{R}}\right)$.

As a step towards proving the conjecture, they introduced the notion of a real number $\alpha$ being approximable, which means that either

1. $\alpha$ is algebraic, or

2. $\alpha$ is transcendental and there exists a family of 0 -variate $\operatorname{SLPs}\left(\Gamma_{n}\right)_{n \in \omega}$ such that $\left|\Gamma_{n}\right|=n^{O(1)}$ and for every $n$ there is no univariate SLP of length at most $n$ such that the polynomial represented by it has a real root between $\alpha$ and the real number represented by $\Gamma_{n}$.

They showed that $\mathbf{P}^{L_{0}} /$ poly $\supset \operatorname{BP}\left(\mathbf{P}_{\mathbb{R}}^{\{\alpha\}}\right)$ if $\alpha \in \mathbb{R}$ is approximable, and that almost every real number is approximable. In addition, they proved that the values of elementary functions at rationals are approximable.

A natural question to ask next is whether or not there exists a nonapproximable transcendental number of higher complexity, e.g., among non-

*Department of Computer Science, University of Tokyo

${ }^{\dagger}$ Research Institute for Mathematical Sciences, Kyoto University 
computable reals. In this light, we investigated the approximability of algorithmically random reals, and showed the following.

Theorem. Every Schnorr random real is approximable.

We prove this by effectivizing Allender et al.'s proof that almost every real number is approximable. Specifically, we construct a c. e. null cover of computable measure that contains all non-approximable reals in $[0,1]$, by exploiting the fact that approximating the complex roots of a given polynomial can be done effectively [3].

In fact, Schönhage's algorithm [3] approximates the roots efficiently, but our proof does not exploit this fact. It remains open whether we can strengthen our claim using notions of resource-bounded randomness. The notion of resourcebounded randomness based on tests has been introduced by Ko [2], and is dependent on two parameters: a complexity class that the null cover belongs to, and an upper bound of the rate at which the amount of information extracted by a null cover from an initial segment of an infinite sequence grows depending on its length. We have been unable to generalize our proof to this setting, because the null cover we constructed does not seem to be even computable, and the rate of information growth is also unclear.

Acknowledgements. This work is based on part of the bachelor thesis of the second author at the University of Tokyo. The authors thank the advisor Hiroshi Imai and his group members for their support and discussions. The work was supported in part by KAKENHI.

\section{References}

[1] E. Allender, P. Bürgisser, J. Kjeldganard-Pedersen, and P. B. Miltersen, On the complexity of numerical analysis, SIAM Journal on Computing, 38 (2009), pp. 1987-2006.

[2] K.-I. Ko, On the notion of infinite pseudorandom sequences, Theoretical Computer Science, 48 (1986), pp. 9-33.

[3] A. Schönhage, The fundamental theorem of algebra in terms of computational complexity, preliminary report, Mathematisches Institut der Universität Tübingen, August 1982. 


\title{
On the Effectively $G_{\delta}$-Decomposable Functions
}

\author{
Takayuki Kihara \\ Japan Advanced Institute of Science and Technology, Japan \\ kihara.takayuki.logic@gmail.com
}

Suppose that $X$ and $Y$ are topological spaces, and $\boldsymbol{\Gamma}$ is a pointclass. A function $f: X \rightarrow Y$ is $\boldsymbol{\Gamma}$-piecewise continuous if there is a countable $\boldsymbol{\Gamma}$-cover $\left\{X_{n}\right\}_{n \in \omega}$ of $X$ such that $f \uparrow X_{n}$ is continuous for every $n \in \omega$. For instance, Dirichlet's nowhere continuous function $\chi_{\mathbb{Q}}$ is obviously $\boldsymbol{\Pi}_{2}^{0}$-piecewise continuous, but not $\boldsymbol{\Pi}_{1}^{0}$-piecewise continuous.

Indeed, Dirichlet's function is $\boldsymbol{\Pi}_{2}^{0}$-2-wise continuous. Here, a function $f$ : $X \rightarrow Y$ is $\boldsymbol{\Gamma}$-n-wise continuous if there is a $\boldsymbol{\Gamma}$-cover $\left\{X_{k}\right\}_{k<n}$ of $X$ such that $f \uparrow X_{k}^{\prime}$ is continuous, where $X_{k}^{\prime}=X_{k} \backslash \bigcup_{j<k} X_{j}$, for every $k<n$. As another example of a $\boldsymbol{\Pi}_{2}^{0}-n$-wise continuous function, it is known in topological dimension theory that there is a $\boldsymbol{\Pi}_{2}^{0}$ - $(n+1)$-wise embedding of $\mathbb{R}^{n}$ into $2^{\omega}$ whereas there is no $\boldsymbol{\Pi}_{2}^{0}$-n-wise embedding of $\mathbb{R}^{n}$ into $2^{\omega}$.

The hierarchies of $\boldsymbol{\Pi}_{1}^{0}$-piecewise continuous functions have been extensively studied in the context of the levels of discontinuity $[1,4,5,8]$, the subhierarchy of Baire-one-star functions [6,7,9], and the mind-change hierarchy $[2,3]$. In this talk, we deal with a proper hierarchy of $\boldsymbol{\Pi}_{2}^{0}$-piecewise continuous functions.

A $\boldsymbol{\Gamma}$-piecewise continuous function is controlled by a conditional branching by a $\boldsymbol{\Gamma}$ formula. The flowchart of this control process is represented as an infinite branching tree $T$ of height 2 , where the root of $T$ is labeled by a $\Gamma$ formula, and each leaf (terminal node) of $T$ is labeled by a partial continuous function. A flowchart for a nested-piecewise continuous function will be represented as a well-founded tree $T \subseteq \omega^{<\omega}$, where every nonterminal node is labeled by a formula, and every terminal node is labeled by a continuous function.
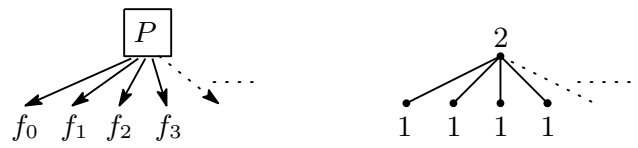

Fig. 1. (left) A realization of $\boldsymbol{\Pi}_{2}^{0}$-piecewise continuity via a $\boldsymbol{\Pi}_{2}^{0}$-condition $P=\left\langle P_{i}\right\rangle_{i \in \omega}$ and continuous functions $\left\langle f_{i}\right\rangle_{i \in \omega}$; (right) The skeleton representing $\boldsymbol{\Pi}_{2}^{0}$-piecewise continuity.

For a well-ordered set $(I,<)$, a sequence $\left\langle P_{i}: i \in I\right\rangle, P_{i} \subseteq X$, is called a $\Gamma$-layer of $Q$ if $Q \subseteq \bigcup_{i \in I} P_{i}$, and there is a $\Gamma$ set $R \subseteq I \times X$ such that $P_{i}=$ $R^{[i]} \backslash \bigcup_{j<i} R^{[j]}$, where $R^{[i]}$ is the $i$-th section of $R$, i.e., $R^{[i]}=\{x \in X:(i, x) \in R\}$.

Definition 1. A skeleton is a pair $\mathbb{S}=(S, \nu)$ of a well-founded tree $S \subseteq \omega^{<\omega}$ and a function $\nu: S \rightarrow \omega_{1}$. A realization of a skeleton $(S, \nu)$ is a collection consisting of $\Lambda=\left\langle P_{\sigma}, f_{\rho}: \sigma \in S, \rho \in S^{\text {leaf }}\right\rangle$ such that

(i) $\left\langle P_{\sigma \frown i}: i \in \operatorname{succ}_{S}(\sigma)\right\rangle$ forms a $\Pi_{\nu(\sigma)}^{0}$-layer of $P_{\sigma}$ for every $\sigma \in S \backslash S^{\text {leaf }}$. 
(ii) $f_{\rho}$ is a $\boldsymbol{\Sigma}_{\nu(\rho)}^{0}$-measurable function with domain $P_{\rho}$ for every leaf $\rho \in S^{\text {leaf }}$.

The set $P_{\langle\rangle}$is called the domain of the realization $\Lambda$, and written as $\operatorname{dom}(\Lambda)$. In this talk, we assume that $\nu(\sigma)=1$ for every leaf $\sigma$ of a skeleton.

Note that for every $x \in \operatorname{dom}(\Lambda)$, there is a unique leaf $\operatorname{TP}_{\Lambda}(x) \in S^{\text {leaf }}$ such that $x \in \bigcap_{\sigma \preceq \mathrm{TP}_{\Lambda}(x)} P_{\sigma}$. Such a leaf $\mathrm{TP}_{\Lambda}(x)$ is said to be the true path of $\Lambda$ at $x$. The realization $\Lambda$ generates a function $f_{\Lambda}: \operatorname{dom}(\Lambda) \rightarrow Y$ by $f_{\Lambda}(x)=f_{\operatorname{TP}_{\Lambda}(x)}(x)$.

Definition 2. Let $\mathbb{S}=(S, \nu)$ be a skeleton. A function $f$ is said to be $\mathbb{S}$-piecewise continuous if there is a realization $\Lambda$ of $\mathbb{S}$ such that $f=f_{\Lambda}$.

The rank of the skeleton $\mathbb{S}=(S, \nu)$ is the least ordinal $\gamma<\omega_{1}$ such that for every $\sigma \in S \backslash S^{\text {leaf }}, \nu(\sigma) \leq \gamma$, and \# $\operatorname{succ}_{S}(\sigma)=\infty$ implies $\nu(\sigma)<\gamma$.

We study the nested-piecewise continuity with respect to a skeleton of rank 2. Clearly, such a continuity is stronger than the $G_{\delta}$-piecewise continuity. Thus, by analyzing the property of a such continuity notion, we can understand the fine structure of the $G_{\delta}$-piecewise continuous functions.

We show that if skeletons $\mathbb{S}_{0}$ and $\mathbb{S}_{1}$ of rank 2 are sufficiently different, then $\mathbb{S}_{0}$-piecewise continuity and $\mathbb{S}_{1}$-piecewise continuity are also different. More precisely, we show that the $\mathbb{S}_{0}$-piecewise notion and the $\mathbb{S}_{1}$-piecewise notion have very different computational power. To see this, we shall introduce the notion of piecewise computability.

Definition 3. A skeleton $(S, \nu)$ is computable if $S$ is a computable well-founded tree, and $\nu: S \rightarrow \omega_{1}^{\mathrm{CK}}$ is computable, where $\omega_{1}^{\mathrm{CK}}$ is represented via some computable coding such as Kleene's system of ordinal notations.

Definition 4. A realization $\Lambda=\left\langle P_{\sigma}, f_{\rho}: \sigma \in S, \rho \in S^{\text {leaf }}\right\rangle$ of a computable skeleton $(S, \nu)$ is computable if there is a computable function $q: S \rightarrow \omega$ such that $q(\sigma)$ is an index of the (lightface) $\Pi_{\nu(\sigma)}^{0}$-layer $\left\langle P_{\sigma i}\right\rangle_{i \in \operatorname{succ}_{S}(\sigma)}$ for every nonterminal node $\sigma \in S$, and $q(\rho)$ is an index of the effectively $\Sigma_{\nu(\rho)}^{0}$-measurable function $f_{\rho}$ for every terminal node $\rho \in S^{\text {leaf }}$.

Recall that $\nu(\rho)$ is supposed to be 1 for every terminal node $\rho \in S^{\text {leaf }}$. Therefore, $f_{\rho}$ must be computable.

Definition 5. Let $\mathbb{S}=(S, \nu)$ be a skeleton. A function $f$ is said to be $\mathbb{S}$-piecewise computable if there is a computable realization $\Lambda$ of $\mathbb{S}$ such that $f=f_{\Lambda}$.

For subsets of topological spaces $A \subseteq X \times Y$ and $B \subseteq Z \times W$, a function $f: A \rightarrow B$ satisfies the Weihrauch condition if there exists a continuous function $p: X \rightarrow Z$ such that every $f(p(x), y)$ is of the form $\langle x, w\rangle$, that is, $f\left[A^{[p(x)]}\right] \subseteq$ $B^{[x]}$ for every $x \in Z$. One of our main theorems can be written as follows:

Theorem 1. Suppose that skeletons $\mathbb{S}_{0}$ and $\mathbb{S}_{1}$ of rank 2 are sufficiently different. Then, there are compact (indeed, $\Pi_{1}^{0}$ ) sets $P \subseteq Q \subseteq 2^{\omega} \times 2^{\omega}$ such that there exists a $\mathbb{S}_{0}$-piecewise computable function $f: Q \rightarrow P$ satisfying the Weihrauch condition via $p=\mathrm{id}$ (indeed, $f \uparrow P=\mathrm{id}$ ), whereas there is no $\mathbb{S}_{1}$-piecewise continuous function $f: Q \rightarrow P$ satisfying the Weihrauch condition. 
In our proof, each skeleton plays a role of priority tree, and the true path $\mathrm{TP}(x)$ in the previous definition plays a role of the true path in the sense of the usual priority argument, and then the usual infinite injury priority argument ensures the difference between two different skeleton-continuity/computability notions.

For instance, consider the following rank 2 skeletons of height 3 :

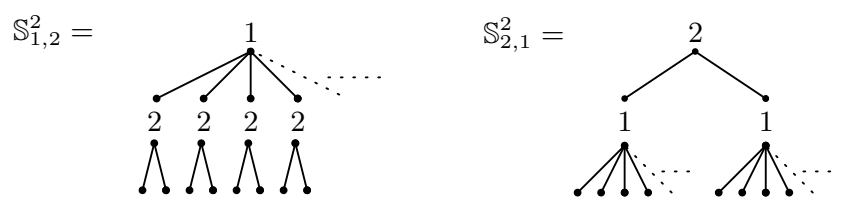

Fig. 2. (left) $S_{1,2}^{n}=\{\langle\rangle,\langle k\rangle,\langle k, m\rangle: k \in \omega, m<n\}, \nu_{1,2}(\langle\rangle)=1$, and $\nu_{1,2}(\langle k\rangle)=2$; (right) $S_{2,1}^{n}=\{\langle\rangle,\langle m\rangle,\langle m, k\rangle: m<n, k \in \omega\}, \nu_{2,1}(\langle\rangle)=2$, and $\nu_{2,1}(\langle m\rangle)=1$.

Our theorem clearly includes the following corollary:

Corollary 1 There is a $\mathbb{S}_{1,2}^{2}$-piecewise computable function which is not $\mathbb{S}_{2,1^{n}}^{n}$ piecewise continuous for any $n \in \omega$. There is a $\mathbb{S}_{2,1}^{2}$-piecewise computable function which is not $\mathbb{S}_{1,2}^{n}$-piecewise continuous for any $n \in \omega$.

Indeed, our main theorem implies much more stronger property. For instance:

Corollary 2 Suppose that $P$ is a $\Pi_{1}^{0}$ subset of $2^{\omega}$ having no computable element. Then, there exists a $\Pi_{1}^{0}$ set $Q \subseteq 2^{\omega}$ with $Q \supseteq P$ such that there is a $\mathbb{S}_{2,1}^{2}$-piecewise computable function $f$ with $Q \subseteq \operatorname{dom}(f)$ and $f[Q] \subseteq P$, and for any $n$, there is no $\mathbb{S}_{1,2}^{n}$-piecewise computable function $f$ with $Q \subseteq \operatorname{dom}(f)$ and $f[Q] \subseteq P$.

\section{References}

1. de Brecht, M., 2013. Levels of discontinuity, limit-computability, and jump operators, preprint.

2. de Brecht, M., Yamamoto, A., 2009. Interpreting learners as realizers for $\Sigma_{2^{-}}^{0}$ measurable functions (extended abstract). Special Interests Group on Fundamental Problems in Artificial Intelligence (SIG-FPAI) 74, 39-44.

3. Freivalds, R., Smith, C. H., 1993. On the role of procrastination in machine learning. Inf. Comput. 107 (2), 237-271.

4. Hemmerling, A., 2008. Hierarchies of function classes defined by the first-value operator. Theor. Inform. Appl. 42 (2), 253-270.

5. Hertling, P., 1996. Topological complexity with continuous operations. J. Complexity $12(4), 315-338$.

6. Matek, A., 2006. A classification of Baire one star functions. Real Anal. Exchange $32,205-212$.

7. O'Malley, R. J., 1977. Approximately differentiable functions: the $r$ topology. Pacific J. Math. 72 (1), 207-222.

8. Pauly, A., 2010. On the (semi)lattices induced by continuous reducibilities. MLQ Math. Log. Q. 56 (5), 488-502.

9. Pawlak, R. J., 2000. On some class of functions intermediate between the class $\mathcal{B}_{1}^{*}$ and the family of continuous functions. Tatra Mt. Math. Publ. 19, 135-144. 


\title{
Exact Function Interval Arithmetic
}

\author{
Michal Konečný ${ }^{1}$ and Jan Duracz ${ }^{2}$ \\ 1 School of Engineering and Applied Science, Aston University, Birmingham, UK, \\ 2 jan@duracz.net
}

We describe a method for implementing exact real arithmetic based on function approximations, building on ideas from iRRAM [6] and Taylor Models [5].

Exact real computation aims to provide a method for programming with real numbers that agrees as much as possible with a mathematician's intuition, minimising the need to consider matters of internal representation and approximation errors introduced by the limitations of computers. In typed languages this is facilitated by a real number type that supports common operations such as the arithmetic field operations $(+,-, \cdot, /)$, computable versions of equality and dis-equality $(=, \neq)$ and order-relations $(<, \leq,>, \geq)$.

In applications that feature intervals, such as decision procedures for inclusion relations between interval expressions [2], e.g.

$$
1-e^{x^{2}}\left(\frac{0.3480242}{1+0.47047 x}-\frac{0.0958798}{(1+0.47047 x)^{2}}+\frac{0.7478556}{(1+0.47047 x)^{3}}\right) \in \frac{2}{\sqrt{\pi}} \int_{0}^{x} e^{-t^{2}} \mathrm{~d} t \pm 0.00005
$$

one needs exact interval computation supported by a real interval type. Beyond interval extensions of the arithmetic operations and order relations, the interval type should support also the refinement relation (), i. e. inverse inclusion of real intervals.

Exact real or interval computation may be realised in a number of ways. We adopt the approach where an exact quantity is approximated by a sequence of approximants that converge to the exact quantity. A real number is approximated by an interval, while an interval is usually approximated by a pair of intervals, one giving an outer bound and the other giving an inner bound on the exact interval. The elements of the sequence are computed on demand until an approximation is obtained that is sufficiently precise to decide a relation or print a sufficient amount of digits. Moreover, the elements of the sequence are computed one by one, using increasing values of a parameter that controls the precision of the computation. This approach was first introduced in iRRAM [6] and is also used in RealLib [4].

Using intervals as approximants has well-known issues, the most significant of which is the dependency problem. The dependency problem occurs when multiple instances of a single number $x$ are approximated by an interval, say $[0, \pi]$, and when the information is lost that those instances of the interval $[0, \pi]$ all approximate the same number. For example, computing $\cos ^{2}(x)+\sin ^{2}(x)$ using interval operations gives $[0,2]$, which is much wider-less informative- than the exact result 1.

One way to reduce the dependency error is to split up the intervals that approximate the affected variables and perform the computation for each segment and then merge all the results. However, this approach often comes at an exponential cost.

We prefer an alternative approach that is based on parametrised intervals, or function intervals, which retain some relational information among variables. For example, the expressions $\sin (x)$ and $\cos (x)$ can be approximated by a pair of representable functions, such as polynomials, that bound the respective functions from below and above. Operations such as squaring and addition are applied pointwise 
to these interval functions to obtain an approximation of $\cos ^{2}(x)+\sin ^{2}(x)$, which contains the constant function 1. Moreover, by increasing parameters such as the polynomial order, the computation gives a sequence of function intervals that converges to the constant function 1 .

This approach has been used in the form of so-called Taylor Models (TMs) [5], in which a function interval is represented by a single polynomial with rational coefficients, except the constant coefficient, which is a rational interval that lends its thickness to the function interval. Due to the fact that TMs have constant width across their domain, they are suitable for approximating exact real functions but not for approximating exact interval functions.

We use the term exact function interval arithmetic for an arithmetic that can arbitrarily closely approximate

- function intervals that are given by pairs of continuous functions over rectangular domains;

- a certain set of operations on such function intervals.

We describe the API and the most important internal aspects of our implementation of an exact function interval arithmetic based on polynomials (available freely from gi thub . org/michalkonecny/ aern). The API operations include integration and evaluation of a range over a sub-box of the domain. The operation that we found most difficult to implement was multiplication. This is because it requires pointwise minimisation and maximisation:

$$
[a, b] \cdot[c, d]=[\min (a c, a d, b c, b d), \max (a c, a d, b c, b d)]
$$

We approximate min and max using Bernstein approximations. Computing Bernstein approximations require multiplication, creating a dependency cycle. To break the cycle, for the Bernstein approximations we use a rough multiplication that works well only for individual polynomials, i. e. for the bounds of the function intervals.

To illustrate the power and limitations of our implementation of exact function interval arithmetic we give several application examples. In particular, we show that the arithmetic, embedded in the theorem prover PolyPaver (freely available from gi thub.com/michalkonecny/polypaver), can be used to prove inclusions such as equation (1). We also demonstrate a direct implementation of the interval Picard operator of Edalat \& Pattinson [3], enabling one to compute tight enclosures of ODE IVPs with uncertain bounds. (Another CCA 2014 talk by our wider team gives details of this version of the interval Picard operator.)

For further information on the arithmetic and its application see the extended abstract [1].

\section{References}

1. Jan Duracz, Amin Farjudian, Michal Konečný, and Walid Taha. Function interval arithmetic. In International Congress on Mathematical Software (ICMS 2014), volume 8592 of LNCS, 2014.

2. Jan Duracz and Michal Konečný. Polynomial function intervals for floating-point software verification. Annals of Mathematics and Artificial Intelligence, 70(4):351-398, April 2014.

3. Abbas Edalat and Dirk Pattinson. A domain-theoretic account of Picard's theorem. LMS Journal of Computation and Mathematics, 10:83-118, 2007.

4. Branimir Lambov. RealLib: An efficient implementation of exact real arithmetic. Mathematical Structures in Computer Science, 17(1):81-98, 2007.

5. Kyoko Makino and Martin Berz. New applications of Taylor model methods. In Automatic Differentiation of Algorithms: From Simulation to Optimization, chapter 43, pages 359-364. Springer, 2002.

6. Norbert Th. Müller. The iRRAM: Exact arithmetic in C++. In Jens Blanck, Vasco Brattka, and Peter Hertling, editors, CCA, volume 2064 of Lecture Notes in Computer Science, pages 222-252. Springer, 2000. 


\title{
Picard Method for Enclosing ODEs with Uncertain Initial Values
}

\author{
Michal Konečný ${ }^{1}$, Jan Duracz ${ }^{2}$, Amin Farjudian ${ }^{3}$, and Walid Taha ${ }^{4}$ \\ 1 School of Engineering and Applied Science, Aston University, Birmingham, UK, \\ 2 jan@duracz.net \\ 3 School of Computer Science, University of Nottingham Ningbo, China \\ ${ }^{4}$ Halmstadt University, Sweden \& Rice University, Houston, Texas, USA
}

We present an algorithm for enclosing solutions of ordinary differential equation (ODE) initial value problems (IVPs) with uncertain initial values:

$$
\boldsymbol{y}^{\prime}=\boldsymbol{f}(\boldsymbol{y}), \boldsymbol{y}(0) \in \boldsymbol{A}
$$

where $\boldsymbol{A} \in \mathbb{I}^{n}$ is a vector of intervals, seen as a box-shaped subset of $\mathbb{R}^{n}$ and $\boldsymbol{f}: \mathbb{R}^{n} \rightarrow \mathbb{R}^{n}$ is a Lipschitz vector field and a solution $\boldsymbol{y}$ has the type $[0, b] \rightarrow \mathbb{R}^{n}$.

Unlike other methods for enclosing solutions of ODE IVPs with uncertain initial values [3,2,4], our algorithm does not assume that the field is differentiable. Also, as with Edalat and Pattinson's method, our algorithm is simple and the proof of its soundness and convergence is closely related to the usual proof of the Picard-Lindelöf theorem.

If $\boldsymbol{A}=\boldsymbol{a}$ is a singleton interval, the equation has a unique solution, which we denote $\boldsymbol{y}_{\boldsymbol{a}}$. In this case, we utilize the interval Picard operator introduced by Edalat and Pattinson [1]:

$$
\begin{array}{ll}
P_{\boldsymbol{F}, \boldsymbol{a}} & : \quad\left([0, b] \rightarrow \mathbb{I}^{n}\right) \rightarrow\left([0, b] \rightarrow \mathbb{I}^{n}\right) \\
P_{\boldsymbol{F}, \boldsymbol{a}}(\boldsymbol{Y})(t)= & \boldsymbol{a}+\int_{0}^{t} \boldsymbol{F}(\boldsymbol{Y}(s)) \mathrm{d} s
\end{array}
$$

where $\boldsymbol{Y}:[0, b] \rightarrow \mathbb{I}^{n}$ is an interval function and $\boldsymbol{F}:\left([0, b] \rightarrow \mathbb{I}^{n}\right) \rightarrow\left([0, b] \rightarrow \mathbb{I}^{n}\right)$ is a interval function extension of the vector field $f$.

Theorem 1 (Edalat \& Pattinson 2007 [1], interval Picard theorem).

If $\boldsymbol{F}$ is a Lipschitz interval extension of the field $\boldsymbol{f}$ in equation (1), $P_{\boldsymbol{F}, \boldsymbol{a}}$ is inclusion isotone and $P_{F, a}\left(\boldsymbol{Y}_{0}\right) \subseteq \boldsymbol{Y}_{0}$, then

$$
\boldsymbol{Y}_{0} \supseteq P_{\boldsymbol{F}, \boldsymbol{a}}\left(\boldsymbol{Y}_{0}\right) \supseteq P_{\boldsymbol{F}, \boldsymbol{a}}^{2}\left(\boldsymbol{Y}_{0}\right) \supseteq \ldots \quad \text { and } \bigcap_{i=0}^{\infty} P_{\boldsymbol{F}, \boldsymbol{a}}^{i}\left(\boldsymbol{Y}_{0}\right)=\boldsymbol{y}_{\boldsymbol{a}} .
$$

We demonstrate on a simple example that this theorem cannot be generalized to uncertain initial value $\boldsymbol{A}$ by simply replacing $\boldsymbol{a}$ with $\boldsymbol{A}$. We instead parametrize this operator so that it can be applied on IVPs without the need to exponentially bisect the space of initial value uncertainty:

$$
\begin{array}{ll}
P_{\boldsymbol{F}} & :\left(\boldsymbol{A} \times[0, b] \rightarrow \mathbb{I}^{n}\right) \rightarrow\left(\boldsymbol{A} \times[0, b] \rightarrow \mathbb{I}^{n}\right) \\
P_{\boldsymbol{F}}(\boldsymbol{Y})(\boldsymbol{a}, t) & =\boldsymbol{a}+\int_{0}^{t} \boldsymbol{F}(\boldsymbol{Y}(\boldsymbol{a}, s)) \mathrm{d} s
\end{array}
$$

Theorem 2 (Interval Picard theorem for flows).

If $P_{\boldsymbol{F}}\left(\boldsymbol{Y}_{0}\right) \subseteq \boldsymbol{Y}_{0}$, then

$$
\boldsymbol{Y}_{0} \supseteq P_{\boldsymbol{F}}\left(\boldsymbol{Y}_{0}\right) \supseteq P_{\boldsymbol{F}}^{2}\left(\boldsymbol{Y}_{0}\right) \supseteq \ldots \quad \text { and } \bigcap_{i=0}^{\infty} P_{\boldsymbol{F}}^{i}\left(\boldsymbol{Y}_{0}\right)=\boldsymbol{y}_{\boldsymbol{A}} .
$$

where $\boldsymbol{y}_{\boldsymbol{A}}$ is the flow of the ODE in (1), defined by $\boldsymbol{y}_{\boldsymbol{A}}(\boldsymbol{a}, t)=\boldsymbol{y}_{\boldsymbol{a}}(t)$ for all $\boldsymbol{a} \in \boldsymbol{A}$. 
Switching from $P_{F, A}$ to the parametrized operator $P_{F}$ is beneficial not only for solving IVPs with uncertain initial value, but also for piece-wise solving IVPs with exact initial value. This is because the initial value for each new step has a small level of uncertainty resulting from the imprecision of the enclosures for the preceding time step.

We propose an ODE solving algorithm enclose-flow based on a direct implementation of the parametrized interval Picard operator $P_{\boldsymbol{F}}$. This algorithm relies on function interval arithmetic. A function interval is a pair of functions of type $\boldsymbol{D} \rightarrow \mathbb{R}$ where $\boldsymbol{D} \subseteq \mathbb{R}^{n}$. A function interval can be also seen as a function of type $\boldsymbol{D} \rightarrow \mathbb{I}$, which appears in equations (2) and (3). A function interval arithmetic makes function intervals first-class objects and provides operations such as point-wise addition or integration, which facilitates computing the operator $P_{F}$.

We do not discuss the implementation of function interval arithmetic but formulate a set of requirements for the arithmetic. These requirements imply the safety of enclose-flow. By extending the requirements we obtain the concept of exact function interval arithmetic. We prove that with an exact arithmetic, enclose-flow can produce enclosures that converge to the exact flow $\boldsymbol{y}_{\boldsymbol{A}}$.

We demonstrate the feasibility of the approach using one of our implementations of function interval arithmetic. (Available freely from github.org/michalkonecny/aern) In particular, we produce very tight enclosures for a spring mass system with initial value uncertainty, an ODE featuring an absolute value, and a version of the Van der Pol system. Moreover, a version of our method, using a different function interval arithmetic, is included in the Acumen tool and language for modeling and rigorous simulation of hybrid dynamical systems. (Available freely from www.acumen-language.org)

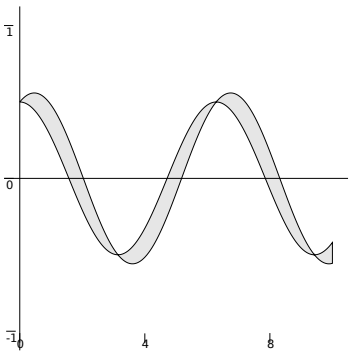

Spring mass with uncertain initial speed

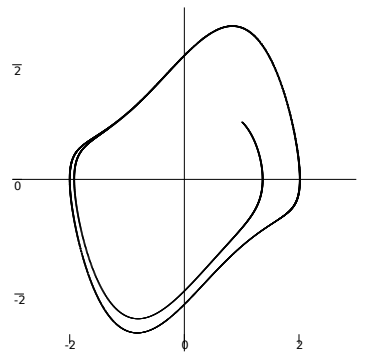

Van der Pol system

\section{References}

1. Abbas Edalat and Dirk Pattinson. A domain-theoretic account of Picard's theorem. LMS Journal of Computation and Mathematics, 10:83-118, 2007.

2. Kyoko Makino and Martin Berz. New applications of Taylor model methods. In Automatic Differentiation of Algorithms: From Simulation to Optimization, chapter 43, pages 359-364. Springer, 2002.

3. Nedialko S. Nedialkov, Kenneth R. Jackson, and George F. Corliss. Validated solutions of initial value problems for ordinary differential equations. Applied Mathematics and Computation, 105(1):21-68, 1999.

4. Andreas Rauh, Eberhard P. Hofer, and Ekaterina Auer. ValEncIA-IVP: A comparison with other initial value problem solvers. Scientific Computing, Computer Arithmetic and Validated Numerics, International Symposium on, 0:36, 2006. 


\title{
Tools for a gentle slope transition From floating point arithmetic to exact real arithmetic
}

\author{
Valérie Ménissier-Morain* \\ Sorbonne Universités, UPMC Univ Paris 06, UMR 7606, LIP6, F-75005, Paris, France \\ CNRS, UMR 7606, LIP6, Pequan Team, F-75005, Paris, France
}

Floating point arithmetic (FPA) is one century old [19] and is effectively used commonly since 60 years. Exact real arithmetic (ERA) appears 40 years ago [9] and has been developed essentially since the end of the 80's [22, 2] (see [11] for a survey) resulting both of the dissatisfaction about FPA results and the sharp increase of material computing power that allows computation ambition.

FPA is essentially a fixed precision arithmetic while ERA adapts the precision of each operation to ensure the desired accuracy of the result. However for a long time, we have had to choose between fast computed, completely wrong, FPA results on the one hand and accurate ERA results obtained too late to be useful on the other hand.

Alternative to FPA such as interval arithmetic (IA) have been designed by mathematicians since the 50's and during the last three decades numerous tools have been designed by computer scientists to reduce the gap between FPA and ERA with two principle directions: evaluate the inaccuracy of the result and try to produce a more accurate result.

\section{Evaluate the inaccuracy of the result}

\section{Analysis of FPA behavior}

In the case where it is impossible to change radically arithmetic in a program, it is however possible with only a few modifications of the code to analyze its behavior as regards to the accuracy of the result.

It is possible to make static analysis, that is to say without execution of the program, with a tool like Fluctuat developed at CEA List $[10,18]$ that detects by abstract interpretation which variable and which piece of code is critical for the accuracy of the result and computes a bound on the error, and it can take assertions into account like interval of possible values for data measures or properties of the result. Fluctuat is a non-free multi-platform application to analyze $\mathrm{C}$ and Ada95 codes.

As an alternative to this static analysis, there exists a tool for dynamic analysis, Cadna, developed in the Pequan Team at UPMC, based on the notion of stochastic arithmetic of the CESTAC method $[21,4]$ to estimate round-off errors and detect numerical instabilities. The idea is to execute two or three times the same instruction with different rounding modes (randomly chosen with probability $1 / 2$ for $\underline{x}$ and $\bar{x}$ ) before next instruction (synchrone evaluation) to see how to propagate round-off errors; for each instruction the significant result will be the common part of the different results. Cadna is an open source library for Fortran and C. There is an old version in Ada, a partial version for BLAS, and now since a few years the SAM version for MPFR multiprecision [12].

Starting from this analysis the programmer is aware of the numerical quality of his result and can try to improve the conditioning of his program.

A survey on rounding errors analysis is given in [5].

\section{Interval arithmetic}

The principle is very simple [16]: use intervals rather than numbers for two reasons: often numerical data are soiled with measure errors thus considering intervals of values seems very quickly natural in scientific computations (50's ans 60 's) and further it has also been used simply to evaluate the importance of round-off errors in floating point computations.

This leads to two approaches. In the first one, intervals are considered as first class objects and for any function $f$ an algorithm describes how to produce interval $[c, d]$ such that $f(x) \in[c, d]$ for all $x \in[a, b]$ (inclusion property) and then

\footnotetext{
*Valerie.Menissier-Morain@upmc.fr
} 
functional analysis, linear algebra, etc. on this objects are developed. This approach is a branch of pure mathematics and is essentially not concerned with computations. In the second one, intervals $[\underline{x}, \bar{x}]$ represent the result of round-off errors of floating-point computations to guarantee that the final mathematical result is in the computed interval. This approach is the minor one but was clearly boosted by IEEE 754 standard.

There is a free $\mathrm{C}++$ interval library in the Boost repository of free peer-reviewed portable $\mathrm{C}++$ libraries [15].

\section{Try to produce a more accurate result}

\section{Error-free transformations and compensated arithmetic}

The starting point of this approach is the remark that the rounding error of addition and multiplication of two fp numbers is itself a fp number with rounding to nearest. Furthermore between 1965 and 1975 explicit algorithms have been designed to compute this error [14, 7, 20]. Algorithms that produce both the fp result and the fp error are called Error-Free Transformations (EFT).

This has been used to double the precision of computations based on these operations during this decade and further since 2005 it is used again in a systematic way to obtain by iterative refinement more accurate results for example for summation, product, dot product, polynomial evaluation, solution of triangular linear system [17, 13]. The idea is to re-inject the rounding error of an operation into the following one or to accumulate rounding errors to produce a corrective term to add to the result of the fp computation to produce a fp final result as accurate as if computed in doubled (or more) working precision and rounded to working precision.

\section{Multiprecision}

The EFT have also been used since 40 years to implement double-double and quad-double arithmetic in Fortran (Brent MP [3], Bayley MPFUN and QD [1]). These packages can be classified into two formats of numbers: multiple-digit format i.e. numbers are represented as a sequence of digits coupled with a single exponent; multiple-component format, i.e. numbers represented as an unevaluated sum of two (resp. four) IEEE double precision numbers. The purpose is, as for FPA, to have a fixed precision all along the computation but numbers have larger representations than fp numbers.

Later multiprecision appeared to allow more powerful and flexible computations. We can cite GMP/MPF since 1996, MPFR [8] since 2000 and its interval version MPFI since 2002 for C language, with interfaces for lots of other languages. Each number has its own precision and the result is correctly rounded to the precision of the target variable, in any of the four IEEE-754 rounding modes. If the programmer chooses convenient precision for each variable he can obtain an accurate result as with an ERA computation. He simply needs to analyze the computation to deduce the required precision everywhere while ERA computes the result without any precision indication.

\section{Almost ERA with Sollya}

Sollya, developed by PEQUAN UPMC team and APICS INRIA team [6], is both an interactive application and a library for safe $\mathrm{C}$ floating-point code development. It is particularly targeted to the automatized implementation of mathematical floating-point libraries. Sollya uses multi-precision arithmetic (MPFR) and interval arithmetic to produce tight and safe results with the precision required by the user.

The printed value is either exact or generally a faithful rounding of the exact value at the working precision. If a faithful rounding cannot sufficiently quickly be computed, a value is printed that was obtained using floating-point approximations without control on the final accuracy. Whenever something is not exact, a warning is systematically printed.

\section{Conclusion}

These few tools show that there's nothing to despair: even if it is not possible to use ERA, there exists a variety of tools to watch over or insure the accuracy of the results with non-ERA packages.

\section{References}

[1] D. H. BAILEY. High-precision software directory. http://crd-legacy.lbl.gov/ dhbailey/mpdist/. 
[2] H. J. Boenm. Constructive Real Interpretation of numerical Programs. In Proceedings of the 1987 ACM conference on Interpreters and Interpretives Techniques (1987).

[3] R. BRent. A fortran multiple precision arithmetic package. ACM Trans. Math. Soft 4 (1978), 57-70.

[4] J.-M. CheSneAuX. L'arithmétique stochastique et le logiciel CADNA. Habilitation à diriger des recherches, UPMC, 1995.

[5] J.-M. Chesneaux, S. Graillat, And F. JÉZÉQuel. Encyclopedia of Computer Science and Engineering, vol. 4. Wiley, 2009, ch. Rounding Errors, p. 2480-2494.

[6] S. Chevillard, M. Joldeş, And C. Lauter. Sollya: An environment for the development of numerical codes. In Mathematical Software - ICMS 2010 (2010), vol. 6327 of Lecture Notes in Computer Science, Springer, pp. $28-31$.

[7] T. J. DekKer. A floating point technique for extending the available precision. Numerische Mathematik 18, 3 (1971), 224-242.

[8] L. Fousse, G. Hanrot, V. Lefèvre, P. Pélissier, and P. Zimmermann. MPFR: A multiple-precision binary floating-point library with correct rounding. ACM Transactions on Mathematical Software 33, 2 (June 2007), 13:1-13:15.

[9] B. Gosper. Continued Fraction Arithmetic. HAKMEM Item 101B, MIT AI MEMO 239, Feb. 1972.

[10] E. Goubault, S. Putot, P. Baufreton, And J. Gassino. Static analysis of the accuracy in control systems: Principles and experiments. In Proceedings of Formal Methods in Industrial Critical Systems (2007), vol. 4916 of $L N C S$.

[11] P. Gowland AND D. LeSTER. A survey of exact arithmetic implementations. In Computability and Complexity in Analysis (2001), vol. 2064 of Lecture Notes in Computer Science, pp. 30-47. 4th International Workshop, CCA 2000, September 2000.

[12] S. Graillat, F. JÉZÉQuel, S. WAng, And Y. Zhu. Stochastic arithmetic in multiprecision. Mathematics in Computer Science 5 (2011), 359-375.

[13] S. GRaillat, P. Langlois, And N. Louvet. Accurate and validated polynomial evaluation in floating point arithmetic. In Algebraic and Numeric Algorithms and Computer-assisted Proofs (2005), no. 5391 in Dagstuhl Seminar.

[14] D. E. Knuth. The Art of Computer Programming: Seminumerical Algorithms, vol. 2. Addison-Wesley, 1969.

[15] G. Melquiond, S. Pion, And H. Brönnimann. Boost interval arithmetic library. http://www.boost.org/doc/libs/1_55_0/libs/numeric/interval/doc/interval.htm.

[16] R. E. Moore, R. B. Kearfott, And M. J. Cloud. Introduction to Interval Analysis. SiAM Press, 2009.

[17] T. Ogita, S. M. Rump, AND S. OISHI. Accurate sum and dot product. SIAM Journal on Scientific Computing 26, 6 (2005), 1955-1988.

[18] S. Putot. Fluctuat. http://www.lix.polytechnique.fr/Labo/Sylvie.Putot/fluctuat.html.

[19] L. Torres y Quevedo. Ensayos sobre automatica-su defmicion. extension teorica de sus aplicaciones. Revista de la Real Academia de Ciencias Exactas, Fisicas y Naturales 12 (1913), 391-418.

[20] G. W. Veltкamp. Algol procedures voor het berekenen van een inwendig product in dubbele precisie. RCInformatie 22, Technische Hogeschool Eindhoven, 1968.

[21] J. Vignes. A stochastic arithmetic for reliable scientific computation. Mathematics ans Computers in Simulation 35 (1993), 233-261.

[22] J. Vuillemin. Exact real computer arithmetic with continued fractions. Research report 760, INRIA, 1987. 


\title{
Decimal on-line Arithmetic for RatIonal Operation in $\mathrm{C}++(D A R I O C++)$
}

\author{
Gregorio de Miguel Casado* \\ Aragon Institute for Research in Engineering (I3A) \\ University of Zaragoza, Spain \\ gmiguel@unizar.es
}

Computing with real numbers has become an all-time topic since the computer was conceived. Then, after the generalization of computers in research tasks, it has also become of interest for a wide-range of fields in which computers provide essential support for the simulation and validation of mathematical models. Nowadays, there exist a wide range of approaches for exact real and rational number computing according to computer abstraction levels: computational models and paradigms[1-3]; general calculation environments (Maple, Mathematica, ...); software libraries $[4,5]$, and also a draft about IEEE P754 for microprocessors [6], to name a few.

This research proposes the library DARIO $\mathrm{C}++$ for exact arithmetic with decimal rationals in fractional positional notation. It extends previous research done with a base-2 prototype for evaluating hardware feasibility issues [7].

Three main ideas motivate this research: finiteness (real world interaction with a computer is merely finite and limited by the features of sensors and actuators as well as memory bounds), coherency (between the computational model and the software and hardware implementation paradigms so that to guarantee feasibility) and flexibility (implementation tradeoffs in terms of memory and time versus accuracy according to the requirements of the final application).

Then, the approach is based in three cornerstones: exact rational numbers (reals can then be defined with nested Dedekind cuts), Type-2 Theory of Effectivity (TTE) and on-line arithmetic operators (left-to-right operation dynamics for addition, subtraction, multiplication, division and comparison) and a barnotation style (flexible operation: exact, fixed length and constrained length).

The following notation defines decimal rational numbers with fixed and periodic mantissas:

Definition 1. (base-10 bar-notation for rationals) Define the notation $\nu_{b, 10}^{d m}: \subseteq \Sigma^{*} \longrightarrow Q$ by:

$\operatorname{dom}\left(\nu_{b, 10}^{d m}\right):=\left\{\iota_{w}(e) \iota_{w}\left(m_{f}\right) \iota_{w}\left(m_{p}\right) \mid e \in \operatorname{dom}\left(\nu_{Z}\right), m_{f} \in \operatorname{dom}\left(\nu_{b, 10}\right)\right.$,

\footnotetext{
* Research supported by the Spanish Government MICINN Project TIN2011-27479C04-01.
} 


$$
\begin{aligned}
& \left.m_{p} \in \operatorname{dom}\left(\nu_{N}\right) \cup\{\lambda\} \backslash\{0\}, \nu_{b, 10}\left(m_{f}\right) \in \pm[0,9)\right\} \\
& \nu_{b, 10}^{d m}(\text { uvw }):=\left\{q=a / b \mid a=10^{e} \Delta \nu_{b, 10}\left(m_{f} m_{p}-m_{f}\right)\right. \text { with } \\
& e=\iota_{u}(u), m_{f}=\iota_{u}(v), m_{p}=\iota_{u}(w), \text { and } b=\{9\}^{p}\{0\}^{f} \text { with } \\
& f=\left|m_{f}\right| \text { and } p=\left|m_{p}\right|, \\
& \text { and the following restrictions: }
\end{aligned}
$$

1. $p>1$.

2. Let $m_{f}=v_{1} v_{2} \ldots v_{f}$ and $m_{p}=w_{1} w_{2} \ldots w_{p}$, then $v_{1} \neq 0$ and $v_{f} \neq w_{p}$.

3. If $m_{p}=u(v)^{*} w$, then $u \neq v, w$.

The restrictions introduced guarantee (1) no redundancy, (2) no superposition of final digits of fixed and periodic mantissas and (3) minimality of the periodic mantissa (no smaller sub-mantissas).

Several strategies for preserving a canonical output coherent with the notation proposed have being developed. Firstly, prior length calculation of both fixed and periodic mantissas can be done. The latter by means of a table with precalculated data for the periodic mantissa lengths [7, Fig.2]. Secondly, signed digit representations and on-line operators can provide an output of contiguous negative and positive digits (or viceversa) which requires an on-the-fly recodification so that to preserve the notation. For this purpose, a Canonical Signed-Digit recoding (CSD) is done for each output digit by means of a automaton ( 7 states). It addresses all the possible recodifications preemptively, that is preserving the previous complementary result in a register. Finally, tracking small submantissas within the periodic mantissa is done by obtaining digit correspondences from a table according to the divisors of the length of the periodic mantissa.

The complexity of the implementation matches previous results [7]. In addition, some preliminary comparisons done with IRRAM $\mathrm{C}++$ outline calculation times more than 20 times slower.

\section{References}

1. M. Braverman: Computing with Real Numbers, from Archimedes to Turing and Beyond, Communications of the ACM, vol. 56(9), pp. 74-78 (2013)

2. A. Bauer, M.H. Escardó and A. Simpson: Comparing Functional Paradigms for Exact Real-Number Computation, Lecture Notes in Computer Science, vol. 2380, pp. 488-500 (2002)

3. A. Bauer, and I. Kavkler: Implementing Real Numbers with Rz, Electronic Notes in Theoretical Computer Science, vol. 202, pp. 365-384 (2008)

4. P. Gowland and D. Lester: A Survey of Exact Arithmetic Implementations, Lecture Notes in Computer Science, vol. 2064, pp. 30-47 (2001)

5. M. Daumas, D. Lester, and C. Muñoz: Verified Real Number Calculations: A Library for Interval Arithmetic, IEEE Transactions on Computers, vol. 58(2), pp. 226-237 (2009)

6. IEEE Microprocessor Standards Committee: P1788 Draft International Standard for Interval Arithmetic and Complete Arithmetic, IEEE P754 (2008)

7. G. de Miguel Casado: Fractional Positional Notation for Exact Rational Arithmetic, In Proceedings of Computability in Europe 2010, pp. 103-112 (2010) 


\title{
$L C_{N}$-MEASURABLE FUNCTIONS
}

\author{
ALEXEY OSTROVSKY
}

By theorem of Jayne and Rogers a function $f: X \rightarrow Y$ between Polish spaces is $\Delta_{2}$-measurable iff it is piecewise continuous (see [1],[4]).

By computable Jayne and Rogers Theorem we can compute from a name of a $\Delta_{2}$-measurable function a name for the same function as a piecewise continuous function [7].

We can use the computable multi-valued function (or relation $R \subset X \times Y$ ) to check how well we can compute a discontinuous function [8],[2],[3].

However, the generalization of theorem of Jayne and Rogers to multivalued functions raises some topological problems for single-valued functions (see Problem 1 and 2 below).

Recall, that a subset $X$ is $L C_{n}$-set iff it can be written as a union of $n$ locally closed in $X$ sets. A set is locally closed if it is an intersection of a closed and an open set.

A single-valued function is function $f: X \rightarrow Y$ is called piecewise continuous iff $X$ is a union of countable many closed sets $X_{i}$ such that each restriction $f \mid X_{i}: X_{i} \rightarrow f\left(X_{i}\right)$ is continuous.

An arbitrary function is $L C_{n}$-measurable iff the preimage of any open set is $L C_{n}$-set.

Obviously, every single-valued $L C_{1}$-measurable function is $\Delta_{2}^{0}$-measurable.

The following problem (see [6]) remains open for $n=1,2, \ldots$

Problem 1. Let $f: X \rightarrow Y$ be an $L C_{n}$-measurable function between arbitrary subsets $X, Y$ of the Cantor set $C$. Is $f$ piecewise continuous?

A single-valued function $f$ is open- $A_{2}$ iff the multi-valued function $f^{-1}$ is $A_{2}$-measurable.

Theorem 1. A continuous open- $A_{2}$ function $f: X \rightarrow Y$ between arbitrary subsets $X, Y \subset C$ is piecewise open ${ }^{1}$.

Since for every closed in $Y$ subset $Y_{1}$ its complement $Y \backslash Y_{1}$ is a countable union of clopen sets, Theorem 1 follows from the following lemma:

Lemma 1. A continuous function $f: X \rightarrow Y$ between $X, Y \subset C$ is open$A_{2}$ iff there is a closed in $Y$ subset $Y_{1}$ which is homeomorphic to a subspace of natural numbers $\boldsymbol{N}$ and such that the restriction $f \mid f^{-1}\left(Y \backslash Y_{1}\right)$ is open.

\footnotetext{
Key words and phrases. Computable discontinuous, $L C_{n}$-measurable, openness, effective openness, multi-valued function, piecewise continuous.

${ }^{1}=$ there are closed $X_{i} \subset X,(i \in \mathbf{N})$ such that each restriction $f \mid X_{i}: X_{i} \rightarrow f\left(X_{i}\right)$ is open.
} 


\section{ALEXEY OSTROVSKY}

Proof. The implication $\Leftarrow$ is obvious; below, we will prove the inverse implication $\Rightarrow$.

Denote

$$
X_{1}=X \backslash f^{-1}\left(\left\{y: f \text { is not open at some point of } f^{-1}(y)\right\}\right)
$$

and

$$
X_{2}=X \backslash X_{1}
$$

Then $f\left(X_{2}\right)$ is a closed in $Y$ subspace homeomorphic to a subset of natural numbers $\mathbf{N}$.

Indeed suppose the contrary ${ }^{2}$ then there is an $S_{1}(y) \subset Y$ with $y_{k} \rightarrow y \in Y$ and $y_{k} \in f\left(X_{2}\right)$.

Take $x_{k} \in f^{-1}\left(y_{k}\right) \cap X_{2}$. Since $x_{k} \notin X_{1}$, we can find an $S_{2}(y) \supset S_{1}(y)$ and open pairwise disjoint neighborhoods $O\left(x_{k}\right)$ that do not intersect $f^{-1}(y)$ and $f^{-1}\left(y_{k, n}\right)$.

It is easy to see that the set $S_{2}(y) \cap f\left(\bigcup_{k=1}^{\infty} O\left(x_{k}\right)\right)=\left\{y_{k}\right\}_{k=1}^{\infty}$ is not an $A_{2}$-set in $S_{2}(y)$. This contradicts the assumption.

It would be interesting to find the computable version of Theorem 1 and the answer to the following problem (about effectively open functions see $[9])$ :

Problem 2. Is every continuous open-LC $C_{n}$ function between Polish spaces piecewise open for $n=2,3, \ldots$ ?

We direct the reader to Proposition 3.2 in [5] for a more detailed discussion of this problem in the case of $n=1$.

\section{References}

[1] S.I. Adyan and P.S. Novikov, On one semicontinuous function, Zap. MPGI 138 (1958), $3-10$, in Russian.

[2] V. Gregoriades, T. Kispeter, A. Pauly: A comparison of concepts from computable analysis and effective descriptive set theory. CoRR abs/1403.7997(2014).

[3] V. Gregoriades, The descriptive set-theoretic complexity of the set of points of continuity of a multi-valued function. Extended Abstract, CCA, Volume 24, EPTCS, (2010) 92-100.

[4] L. Motto Ros, B. Semmes, A New Proof of a Theorem of Jayne and Rogers, Real Analysis Exchange 35(1), (2009) 195-204.

[5] A. Ostrovsky, Generalization of sequences and convergence in metric spaces, Topology Appl. 171(2014), 63-70.

[6] A. Ostrovsky, Constructible-measurable functions are countably continuous, (2014), submitted for publication.

[7] A. Pauly and M. de Brecht, Non-deterministic computation and the Jayne Rogers Theorem, Proceedings of DCM 2012, Electronic Proceedings in Theoretical Computer Science 143, 2014.

[8] K. Weihrauch, Computable Analysis, Springer (2000).

[9] M. Ziegler, Effectively Open Real Functions, Journal of Complexity Volume 22, Issue $6,(2006)$ 827-849.

\footnotetext{
2 about $S_{1}(y)$ and $S_{2}(y)$ see [5].
} 


\title{
Computability for Basic Quantum Mechanics based on the Hilbert Lattice (extended abstract)
}

\author{
Martin Pape * \\ Fachbereich 4 Mathematik, TU Darmstadt \\ martin.pape@gmx.de
}

Classically there are two equivalent formulations of quantum mechanics. In the functional analytic formulation [9], which is useful for computations, observables are identified with self-adjoint operators. In the logico-algebraic formulation [3,7], which is conceptually appealing, the central object is the Hilbert lattice, which is identified with $\{0,1\}$-valued observables. The first formulation has already been studied in the framework of Weihrauch's Type Two Effectivity (TTE) $[10]$ in $[4,11]$, whereas computability for the latter has, so far, not been considered in the literature.

TTE can be rephrased in terms of constructive analysis inside the function realizability topos $\mathbf{R T}\left(\mathcal{K}_{2}\right)[2,5]$ or rather its restriction to effective morphisms, the so called Kleene-Vesley topos $\mathcal{K} \mathcal{V}$ [8]. The category AdmRep of admissible representations of spaces and continuous(ly realizable) maps between them, which forms the backbone of TTE, can be abstractly characterized within $\mathbf{R T}\left(\mathcal{K}_{2}\right)$. It is equivalent to a (fairly) small full subcategory $\mathbf{Q C B} \mathbf{B}_{0}$ of the category of topological spaces and continuous maps, namely the one on $T_{0}$ quotients of countably based $T_{0}$ spaces [1]. Besides being cartesian closed and closed under regular subobjects, i.e. classical subobjects, it also contains all complete separable metric spaces and, accordingly, is a natural place for the functional analytic approach to quantum mechanics.

In our account, however, we provide a notion of computability for the logicoalgebraic approach. Due to the closure properties of AdmRep and the fact that it hosts the Sierpiński space $\Sigma$, it also hosts the Hilbert lattice $\mathcal{L}$ of closed subspaces of the Hilbert space $\mathcal{H}$, which appears as a regular subobject of $\Sigma^{\mathcal{H}}$. Notice that a closed subspace $P$ of $\mathcal{H}$ is represented by the continuous map $p \in \Sigma^{\mathcal{H}}$ with $P=p^{-1}(\perp)$, i.e. somewhat surprisingly $\perp \in \Sigma$ plays the role of "true". As a consequence the natural order induced by $\Sigma$ on $\mathcal{L}$ is opposite to subset inclusion as considered usually.

Let $\mathcal{I}$ be the unit interval $[0,1]$ with the lower topology. In AdmRep we will identify the space $S t$ of quantum states as the regular, subobject of $\mathcal{I}^{\mathcal{L}}$

*joint work with Thomas Streicher 
consisting of those $s$ which validate the conditions

(S1) $s(0)=0$ and $s(\mathcal{H})=1$

(S2) $s(P \vee Q)=s(P)+s(Q)$ whenever $P \perp Q$, i.e. $\forall x \in P . \forall y \in Q .\langle x \mid y\rangle=0$

since $s$ is continuous and thus preserves infima of decreasing $\omega$-chains.

By the spectral theorem for self-adjoint operators on $\mathcal{H}$, quantum observables correspond to projection valued measures on $\mathbb{R}$, i.e. certain maps from the set $\mathfrak{B}(\mathbb{R})$ of Borel subsets of $\mathbb{R}$ to $\mathcal{L}$. But since $\mathfrak{B}(\mathbb{R})$ does not live in AdmRep we have to restrict to a generating subcollection. It turns out that the object $\mathcal{C}(\mathbb{R})$ of closed subsets of $\mathbb{R}$ is a good choice for this purpose since observables can be characterized as those $o \in \mathcal{L}^{\mathcal{C}(\mathbb{R})}$ which validate the conditions

(O1) o commutes with finite meets and joins

(O2) $o(A) \perp o(B)$ whenever $A \cap B=\emptyset$

since $o$ is continuous and thus preserves infima of decreasing $\omega$-chains.

\section{References}

[1] I. Battenfeld, M. Schröder, and A. Simpson. A convenient category of domains. In L. Cardelli, M. Fiore, and G. Winskel, editors, Computation, Meaning and Logic, Articles dedicated to Gordon Plotkin, page 34pp. Electronic Notes in Computer Science, 2007.

[2] A. Bauer. The Realizability Approach to Computable Analysis and Topology. PhD thesis, School of Computer Science, Carnegie Mellon University, Pittsburgh, 2000.

[3] G. Birkhoff and J. von Neumann. The logic of quantum mechanics. Annals of Mathematics, 37:823-843, 1936.

[4] V. Brattka and R. Dillhage. Computability of the spectrum of self-adjoint operators. j-jucs, 11(12):1884-1900, dec 2005.

[5] P. Lietz. From Constructive Mathematics to Computable Analysis via the Realizability Interpretation. PhD thesis, Fachbereich Mathematik, TU Darmstadt, Darmstadt, 2004.

[6] M. Pape and T. Streicher. Computability for basic quantum mechanics based on the Hilbert lattice. http://www.mathematik.tu-darmstadt.de/ streicher/PaSt.pdf.

[7] P. Pták and S. Pulmannová. Orthomodular structures as quantum logics. Fundamental theories of physics. Kluwer Academic Publishers, 1991.

[8] J. Van Oosten. Realizability: An Introduction to Its Categorical Side. Studies in Logic and the Foundations of Mathematics. Elsevier, 2008.

[9] J. von Neumann. Mathematische Grundlagen der Quantenmechanik. Springer Verlag, Berlin, Germany, 1932.

[10] K. Weihrauch. Computable Analysis. Springer, Berlin, 2000.

[11] K. Weihrauch and N. Zhong. Computing Schrödinger propagators on type-2 Turing machines. Journal of Complexity, 22, 2006. 


\title{
A survey on the strength of Ramsey's Theorem
}

\author{
Tahina Rakotoniaina \\ Department of Mathematics and Applied Mathematics \\ University of Cape Town, South Africa \\ and \\ Faculty of Computer Science \\ Universität der Bundeswehr München, Germany \\ fenoira@gmail.com
}

April 5, 2014

We study the computational content of Ramsey's Theorem with respect to Weihrauch reducibility. The jump operator (i.e. the limit map) is one of the main tools to test the strength of a theorem. For any positive number $n$, the $n$-th jump operator (i.e. the composition of $n$ limit maps) is $\Sigma_{n+1}^{0}$-effectively measurable in the Borel hierarchy and complete with respect to Weihrauch reducibility. In many cases, one can find nice characterizations of the parallelization of a theorem, which combines countably many copies of the respective statement. One of the main results is the following: The $l$-th jump of Weak König's Lemma is equivalent to the parallelization of Ramsey's Theorem of size $l$ and $k$ colors, for any given positive number $l$ and $k$. It follows from this result that increasing the number of colors is not powerful enough to climb to the next level of the Borel hierarchy unlike the case of increasing the size. This main result could be proved by showing inductively that Ramsey's Theorem of size $l+1$ and $k$ colors $\left(\mathrm{RT}_{l+1, k}\right)$ can be computed by the jump of Weak König's Lemma composed with $\mathrm{RT}_{l, k}$.

Given Ramsey's Theorem of size $l$ and $k$ colors, we consider its jump $\left(\mathrm{RT}_{l, k}^{\prime}\right)$, its parallelization $\left(\widehat{\mathrm{RT}_{l, k}}\right)$ and itself of size increased by one $\left(\mathrm{RT}_{l+1, k}\right)$ and compare their strength with each other. One of the main techniques in this study is to consider suitable uniform versions of Ramsey's Theorem. Examples are, the Stable Ramsey Theorem in which only stable colorings are allowed and the Colored Ramsey Theorem in which one has an information about the color of each infinite homogeneous set. It can be shown that the jump of the Colored Ramsey Theorem of size $l$ and $k$ color is equivalent to the Stable Ramsey Theorem of size $l+1$ and $k$ color. Ramsey's Theorem of size one $\left(\mathrm{RT}_{1, k}\right)$ is equivalent to the Bolzano Weierstraß Theorem $\left(\mathrm{BWT}_{k}\right)$. However, one can show that even the product of the limit map and the jump of $\mathrm{RT}_{1, k}$ cannot compute the jump of $\mathrm{BWT}_{k}$. To mention more on separation results: one can easily separate two Ramsey's Theorems of different sizes unlike the case of two Ramsey's Theorems of different colors.

The summary of the results is given by the following picture: 


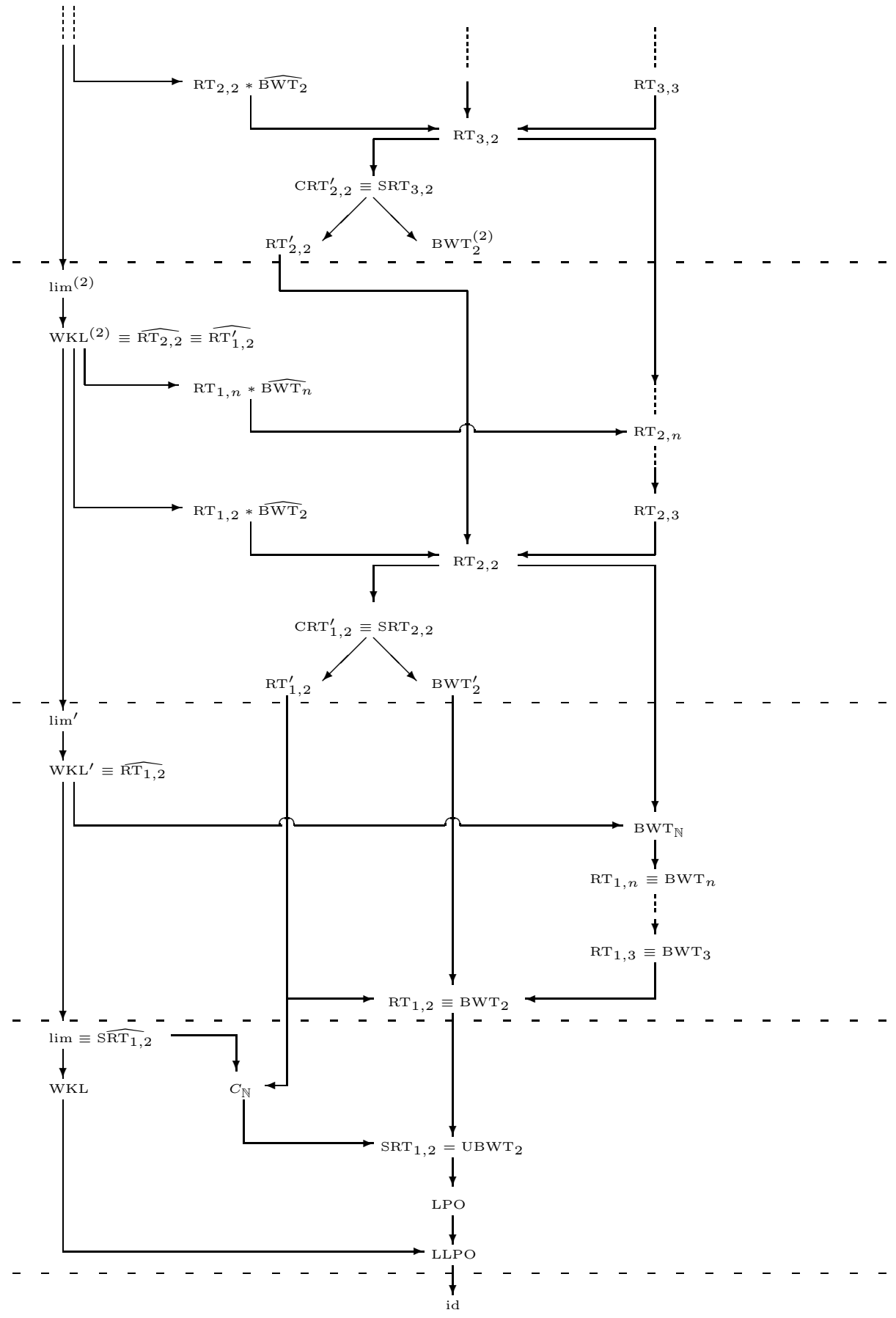




\section{References}

[1] Vasco Brattka, Matthew de Brecht, and Arno Pauly. Closed choice and a uniform low basis theorem. Annals of Pure and Applied Logic, 163:986$1008,2012$.

[2] Vasco Brattka and Guido Gherardi. Effective choice and boundedness principles in computable analysis. The Bulletin of Symbolic Logic, 17(1):73-117, 2011.

[3] Vasco Brattka and Guido Gherardi. Weihrauch degrees, omniscience principles and weak computability. The Journal of Symbolic Logic, 76(1):143-176, 2011.

[4] Vasco Brattka, Guido Gherardi, and Alberto Marcone. The BolzanoWeierstrass theorem is the jump of weak Konnig's lemma. Annals of Pure and Applied Logic, 163:623-655, 2012.

[5] Peter A. Cholak, Carl G. Jockusch, and Theodore A. Slaman. On the strength of Ramsey's theorem for pairs. The Journal of Symbolic Logic, 66(1):1-55, 2001.

[6] François G. Dorais, Damir D. Dzhafarov, Jeffry L. Hirst, Joseph R. Mileti, and Paul Shafer. On the uniform relationships between combinatorial problems. Transactions of the American Mathematical Society, to appear. Preliminary version http://arxiv.org/abs/1212.0157.

[7] Carl G. Jockusch. Ramsey's theorem and recursion theory. The Journal of Symbolic Logic, 37(2):268-280, 1972.

[8] Paulo Oliva and Powell Thomas. A constructive interpretation of Ramsey's theorem via the product of selection functions. Mathematical Structures in Computer Science, to appear. Preliminary version http://arxiv.org/abs/1204.5631.

[9] Alexander P. Kreuzer. The cohesive principle and the Bolzano-Weierstrass principle. Mathematical Logic Quarterly, 57:292-298, 2011.

[10] D. Seetapun and T. Slaman. On the strength of Ramsey's theorem. Notre Dame Journal of Formal Logic, 36(4):570-582, 1995. 


\section{Weihrauch degrees of finding equilibria in sequential games}

\author{
Stéphane Le Roux \\ Department of Mathematics \\ Technische Universität Darmstadt, Germany \\ leroux@mathematik.tu-darmstadt.de
}

\author{
Arno Pauly \\ Computer Laboratory \\ University of Cambridge, United Kingdom \\ Arno.Pauly@cl.cam.ac.uk
}

We continue the investigation of the Weihrauch degrees of operations mapping games to their equilibria started in [10]. There, finding pure and mixed Nash equilibria in two-player games with finitely many actions in strategic form were classified. In the present work, we consider infinite sequential games played by any countable number of players. The best-known example of such games are Gale-Stewart games [4], which are two-player win/loose games. As such, Borel determinacy [8] in principle falls into the scope of this research, although we will be concerned only with determinacy for much smaller pointclasses.

One motivation for this line of inquiry is the general stance that solution concepts in game theory can only be convincing if the players are capable of (at least jointly) computing them taken e.g. in [11]. Even if we allow for some degree of hypercomputation, or are, e.g., willing to tacitly replace actually attaining a solution concept by some process (slowly) converging to it, we still have to reject solution concepts with too high a Weihrauch degree.

The results for determinacy of specific pointclasses that we provide are a refinement of results obtained in reverse mathematics by Nemoto, MedSalem and Tanaka [9]; the first is also a uniformization of a result by CENZER and REMmeL [2]. For some represented pointclass $\Gamma$, let $\mathrm{NE}_{\Gamma}: \Gamma \rightrightarrows\{0,1\}^{\mathbb{N}}$ be the map taking a $\Gamma$-subset $A$ of Cantor space to a (suitably encoded) Nash equilibrium in the sequential two-player game with alternating moves where the first player wins if the induced play is in $A$, and the second player wins otherwise. Let $\mathcal{A}$ be the closed subsets of Cantor space, and $\mathfrak{D}:=\left\{U \backslash U^{\prime} \mid U, U^{\prime} \in \mathcal{A}\right\}$. Our results are:

Theorem 1. $\mathrm{NE}_{\mathcal{A}} \equiv_{W} \mathrm{C}_{\{0,1\}^{\mathbb{N}}}$ and $\mathrm{NE}_{\mathfrak{D}} \equiv_{W} \mathrm{C}_{\{0,1\}^{\mathbb{N}}} \star \lim$.

We have two remarks. One, by combining the preceding theorem with the main result of [1], we find that $\mathrm{NE}_{\mathfrak{D}}$ is equivalent to the Bolzano-Weierstrass-Theorem. This may be a bit unexpected in particular seeing that $\mathrm{C}_{\{0,1\}^{\mathbb{N}}} \star \lim$ is not (yet) known to contain a plethora of mathematical theorems (unlike, e.g., $\mathrm{C}_{\{0,1\}^{\mathbb{N}}}$ ). Two, we already need to use a limit operator in order to move up one level of the difference hierarchy - rather than being able to move up one level in the Borel hierarchy as one may have expected naively. Thus, this observation may complement Harvey Friedman's famous result [3] that proving Borel determinacy requires repeated use of the axiom of replacement.

Another group of results is based on inspecting the various results extending Borel determinacy to more general classes of games (and solution concepts) in $[5,6,7]$. If we instantiate these generic results with specific determinacy version as above, we can prove for some of them that they are actually optimal w.r.t. Weihrauch reducibility. We shall state two such classifications explicitly. 
Consider two-player sequential games with finitely many outcomes, where each player has some acyclic preference relation over the outcomes. For any upper set of outcomes w.r.t. some player's preference let the corresponding set of plays be open or closed. Let $\mathrm{NE}_{\mathcal{O} \cup \mathcal{A}}^{f o}$ be the operation taking such a game (suitably encoded) and producing a Nash equilibrium. Then:

Theorem 2. $\mathrm{NE}_{\mathcal{O} \cup \mathcal{A}}^{\text {fo }} \equiv_{W} \mathrm{C}_{\{0,1\}} \times$ LPO* $^{*}$

Next, we restrict the aforementioned class of games to abstract zerosum games, that is, games where the preferences of one player are the inverse of the preferences of the other player. Those games will have subgame-perfect equilibria, and we let $\mathrm{SPE}_{\mathcal{O} \cup \mathcal{A}}^{a b s z}$ be the operation mapping such games to a subgame-perfect equilibrium.

Theorem 3. $\operatorname{SPE}_{\mathcal{O} \cup \mathcal{A}}^{a b s z} \equiv_{W} \lim$

Various further classifications can be obtained, and adhere to the scheme that algebraic combinations of very common Weihrauch degrees appear, which is already exhibited by our examples above.

\section{References}

[1] Vasco Brattka, Guido Gherardi \& Alberto Marcone (2012): The Bolzano-Weierstrass Theorem is the Jump of Weak König's Lemma. Annals of Pure and Applied Logic 163(6), pp. 623-625, doi:10.1016/j.apal.2011.10.006. Also arXiv:1101.0792.

[2] Douglas Cenzer \& Jeffrey Remmel (1992): Recursively presented games and strategies. Mathematical Social Sciences 24(2-3), pp. 117 - 139, doi:http://dx.doi.org/10.1016/0165-4896(92)90059-E.

[3] Harvey Friedman (1971): Higher set theory and mathematical practice. Annals of Mathematical Logic 2(3), pp. 325-357, doi:10.1016/0003-4843(71)90018-0.

[4] D. Gale \& F.M. Stewart (1953): Infinite games with perfect information. In: Contributions to the theory of games, Annals of Mathematical Studies 28, Princeton University Press, pp. 245-266.

[5] Stéphane Le Roux (2013): Infinite Sequential Nash Equilibria. Logical Methods in Computer Science $9(2)$.

[6] Stéphane Le Roux (2014): From winning strategy to Nash equilibrium. Mathematical Logic Quarterly. (to appear, cf. arXiv 1203.1866).

[7] Stéphane Le Roux \& Arno Pauly (2014): Infinite sequential games with real-valued payoffs. arXiv:1401.3325.

[8] Donald A. Martin (1975): Borel Determinacy. Annals of Mathematics 102(2), pp. pp. 363-371. Available at http://www.jstor.org/stable/1971035.

[9] Takako Nemoto, MedYahya Ould MedSalem \& Kazuyuki Tanaka (2007): Infinite Games in the Cantor space and subsystems of second order arithmetic. Mathematical Logic Quarterly 53(3), pp. 226-236.

[10] Arno Pauly (2010): How Incomputable is Finding Nash Equilibria? Journal of Universal Computer Science 16(18), pp. 2686-2710, doi:10.3217/jucs-016-18-2686.

[11] Arno Pauly (2012): Computable Metamathematics and its Application to Game Theory. Ph.D. thesis, University of Cambridge. 


\title{
Characterising classes of hyperprojective qcb-spaces
}

\author{
Matthias Schröder* \\ Kurt Gödel Research Center, University of Vienna
}

Qcb-spaces [6] play an important role in Computable Analysis. They form the class of sequential spaces which can be appropriately handled by Weihrauch's Type Two Model of Effectivity (TTE) [7]. The category $Q \mathrm{CB}_{0}$ of qcb-spaces satisfying the $T_{0^{-}}$ property and continuous functions has excellent closure properties $[1,3]$ : It is cartesian closed and has countable limits and countable co-limits.

We will characterise some subclasses of qcb-spaces defined by being the smallest full subcategory of $\mathrm{QCB}_{0}$ containing certain spaces and having certain closure properties. This investigation is motivated by a recent observation by M. de Brecht who showed that any full subcategory of $\mathrm{QCB}_{0}$ that contains the Sierpiński space $\mathbb{S}$ and is closed under forming function spaces, countable limits, and countable co-limits in $\mathrm{QCB}_{0}$ contains a homeomorphic copy of any hyperprojective qcb-space.

\section{Hyperprojective qcb-spaces}

Qcb-spaces can be classified using methods of Descriptive Set Theory [2] as follows. Given a family $\boldsymbol{\Gamma}$ of subsets of the Baire space $\mathbb{N}^{\mathbb{N}}$, a qcb-space $X$ is called $\boldsymbol{\Gamma}$-representable [5], if $X$ has an admissible representation $\delta$ such that the set

$$
E Q(\delta):=\{\langle p, q\rangle \mid p, q \in \operatorname{dom}(\delta) \wedge \delta(p)=\delta(q)\}
$$

is in $\boldsymbol{\Gamma}$. Families $\boldsymbol{\Gamma}$ of interest are the family $\mathbf{P}$ of projective subsets of $\mathbb{N}^{\mathbb{N}}$ and the family $\mathbf{H P}$ of countably hyperprojective subsets. The latter is the smallest family that contains all opens subsets of $\mathbb{N}^{\mathbb{N}}$ and is closed under complement, projection and countable union. Qcb-spaces that are HP-representable (or $\mathbf{P}$-representable) are called hyperprojective (resp. projective). From [5] we know:

Theorem 1 ([5]) The category $\mathrm{QCB}_{0}(\mathbf{H P})$ of hyperprojective qcb-spaces is the smallest (up to homeomorphic equivalence) full subcategory $\mathrm{C}$ of $\mathrm{QCB}_{0}$ that contains the Sierpiński space $\mathbb{S}$ and is closed under forming function spaces, countable limits, and countable co-limits in $\mathrm{QCB}_{0}$.

Remember that a category $\mathrm{C}$ has countable limits iff one can form countable products and equalisers in C. Similarly, $\mathrm{C}$ has countable co-limits iff one can form countable sums and co-equalisers in C. Forming equalisers in $\mathrm{QCB}_{0}$ amounts to forming subspaces and forming co-equalisers in $\mathrm{QCB}_{0}$ amounts to forming $T_{0}$-quotient spaces [3]. In certain subcategories $C$ of $\mathrm{QCB}_{0}$ this is not the case anymore, as we will see later.

*email: schroem4@univie.ac.at 


\section{Results}

First we improve Theorem 1 by observing that we only need closure under forming function spaces, countable products and $T_{0}$-quotients to get every hyperprojective qcbspace.

Theorem 2 The category $\mathrm{QCB}_{0}(\mathbf{H P})$ of hyperprojective qcb-spaces is the smallest (up to homeomorphic equivalence) full subcategory $\mathrm{C}$ of $\mathrm{QCB}_{0}$ that contains $\mathbb{S}$ and is closed under forming function spaces, countable products, and co-equalisers in $\mathrm{QCB}_{0}$.

If $\mathbb{N}$ is an object of a full cartesian closed subcategory $C$ of $Q_{C} B_{0}$, then function spaces and products in $\mathrm{C}$ can be shown to be constructed as in $\mathrm{QCB}_{0}$. Hence:

Proposition 3 The category $\mathrm{QCB}_{0}(\mathbf{H P})$ of hyperprojective qcb-spaces is the smallest (up to homeomorphic equivalence) full countably cartesian closed subcategory $\mathrm{C}$ of $\mathrm{QCB}_{0}$ that contains $\mathbb{S}$ and $\mathbb{N}$ and inherits co-equalisers from $\mathrm{QCB}_{0}$.

By a countably cartesian closed category we mean a category that has countable products and function spaces with the usual properties.

If we replace inheritance of co-equalisers by existence, then we can only construct replete spaces. A qcb $0^{-s p a c e} X$ is replete, if for every qcb $_{0}$-space $Y$ such that $\mathbb{S}^{Y}$ is homeomorphic to $\mathbb{S}^{X}$ there is some continuous injection $j: Y \rightarrow X$ such that $\mathbb{S}^{j}: \mathbb{S}^{X} \rightarrow$ $\mathbb{S}^{Y}$ is a homeomorphism. Any sober and thus any Hausdorff qcb-space is replete.

Theorem 4 The smallest (up to homeomorphic equivalence) full cartesian closed $\mathrm{C}$ of $\mathrm{QCB}_{0}$ that contains $\mathbb{S}$ and $\mathbb{N}$ and has countable products and co-equalisers is the category of replete hyperprojective qcb-spaces.

If we drop the Sierpiński space $\mathbb{S}$, we obtain all quasi-zero-dimensional hyperprojective spaces. A quasi-zero-dimensional qcb-space [4] is a qcb-space that arizes as the sequentialisation of some zero-dimensional space. Analogously, a quasi-normal qcbspace is a qcb-space that arizes as the sequentialisation of some normal space. Any quasi-zero-dimensional space is quasi-normal, and any quasi-normal space is Hausdorff.

Proposition 5 The smallest (up to homeomorphic equivalence) full cartesian closed subcategory $\mathrm{C}$ of $\mathrm{QCB}_{0}$ that contains $\mathbb{N}$ and has countable products and co-equalisers is the category $\mathrm{QZ}(\mathbf{H P})$ of quasi-zero-dimensional hyperprojective qcb-spaces.

Surprisingly, one can replace existence of co-equalisers by existence of equalisers. Remember that a category is countably complete iff it has countable products and equalisers iff it has all countable limits.

Theorem 6 The smallest (up to homeomorphic equivalence) full cartesian closed and countably complete subcategory $\mathrm{C}$ of $\mathrm{QCB}_{0}$ containing $\mathbb{N}$ is the category $\mathrm{QZ}(\mathbf{H P})$ of quasi-zero-dimensional hyperprojective qcb-spaces.

Theorem 6 and Proposition 5 hold analogously for quasi-normal hyperprojective spaces: one only has to add the condition on $C$ that $C$ contains the Euclidean space $\mathbb{R}$. 
Theorem 7 The category $\mathrm{QN}(\mathbf{H P})$ of quasi-normal hyperprojective qcb-spaces is equal to:

(1) the smallest (up to homeomorphic equivalence) full cartesian closed subcategory $\mathrm{C}$ of $\mathrm{QCB}_{0}$ that contains $\mathbb{N}$ and $\mathbb{R}$ and has countable products and co-equalisers;

(2) the smallest (up to homeomorphic equivalence) full cartesian closed and countably complete subcategory $\mathrm{D}$ of $\mathrm{QCB}_{0}$ that contains $\mathbb{N}$ and $\mathbb{R}$.

Similar results can be obtained for projective qcb-spaces by considering only finite products rather than countable products.

\section{References}

[1] Escardó, M., Lawson, J., Simpson, A.: Comparing Cartesian closed Categories of Core Compactly Generated Spaces. Topology and its Applications 143 (2004), $105-145$.

[2] Kechris, A.S.: Classical Descriptive Set Theory. Springer, New York, (1995).

[3] Schröder, M.: Admissible representations for continuous computations. $\mathrm{PhD}$ thesis, Fachbereich Informatik, FernUniversität Hagen (2003).

[4] Schröder, M.: A Note on Closed Subsets in Quasi-zero-dimensional Qcb-spaces. Journal of Universal Computer Science 16,18 (2010), 2711-2732.

[5] Schröder, M., Selivanov, V.: Hyperprojective Hierarchy of Qcb-spaces. CiE 2014, LNCS, to appear. Available as: arXiv:1404.0297

[6] Simpson, A.: Towards a Convenient Category of Topological Spaces. Proc. Thirteenth ALGI Workshop (2003).

[7] Weihrauch, K.: Computable Analysis. Springer, Berlin (2000). 


\title{
True orbit simulations of dynamical systems for validating molecular dynamics simulations
}

\author{
Christoph Spandl \\ Computer Science Department, Universität der Bundeswehr München \\ D-85577 Neubiberg, Germany \\ christoph.spandl@unibw.de
}

\begin{abstract}
Due to respectable power of computers nowadays, molecular dynamics simulation is meanwhile state-of-the-art practice in academic and industrial research [1]. The simulations are ranging from liquids, electrolytes to proteins and DNA. Standard molecular dynamics simulation techniques are based on classical Newtonian mechanics, with corrections in the potentials accounting for quantum mechanical effects. From a numerical analysis point of view, the method consists in integrating a system of ordinary differential equations with a more or less huge amount of degrees of freedom. The complexity of some classes of systems to simulate, e.g. proteins, lead to the development of simulation software packages. This situation in turn raises the question of validation [6]. Two aspects of validating molecular dynamics simulations are illuminated here. The first concerns the validity of numerical integration schemes. The second concerns statistical effects, since simulation runs are of finite length.

Molecular dynamics integration schemes are based on Newton's second law:

$$
\vec{F}=m \cdot \vec{a} \text {. }
$$

Typically, an integration scheme is obtained by discretizing the above equation. A widespread algorithm in use is the Verlet method. Despite the fact that the Verlet method only approximates the true solution of the ODE, is has some pleasant properties inherited from the original equations of motion. Actually the Verlet method is a discrete dynamical system in its own right. The pleasant property is that the system is symplectic [2].

The present work is concerned with two aspects of validation. The first aspect deals with the problem that the equations of motion in molecular dynamics simulations, and consequently also in the obtained discrete dynamical systems, are typically chaotic. As already examined in the one dimensional case [5], chaotic behavior leads, when iterating the (discrete) equations of motion, asymptotically to a constant loss of significant bits per iteration step in the state space variables. Thus, using standard IEEE-754 floating-point arithmetic for iteration, as typically is done, rounding errors overwhelm the dynamics even after short iteration times. The obvious question which arises here is "What makes molecular dynamics work?" in practice [4]. Some researches speculate that shadowing
\end{abstract}


justifies the methods in use. In this work, discrete dynamical systems are iterated using exact real arithmetic software: the iRRAM package [3]. This allows one to compute true orbits and compare them with pseudo orbits obtained from standard techniques. So, the iRRAM acts, in some sense, as at tool for validating molecular dynamics simulation methods. The second aspect concerns the statistics of simulation results. Typically, in a molecular dynamics simulation, time averages of the quantities of interest are computed. However, simulation runs are only of finite length and the question arises whether the system reached equilibrium so that the computed quantities are reliable. The present work compares true orbits with pseudo orbits with respect to equilibration and quality of statistics.

The results show that there are indeed differences in the statistics of certain quantities in comparison of true orbits to pseudo orbits. The approach presented here may enable researchers to promote the process of molecular dynamics simulation validation. However, the results seem to be complex and further investigations are needed.

\section{References}

1. Michael P. Allen and Dominic J. Tildesley. Computer Simulation of Liquids. Clarendon Press, Oxford, 1989.

2. Ernst Hairer, Christian Lubich, and Gerhard Wanner. Geometric Numerical Integration. Springer-Verlag, Berlin, Heidelberg, 2. edition, 2006.

3. Norbert Th. Müller. The iRRAM: Exact arithmetic in $\mathrm{C}++$. In Jens Blanck, Vasco Brattka, and Peter Hertling, editors, Computability and Complexity in Analysis, volume 2064 of Lecture Notes in Computer Science, pages 222-252, Berlin, 2001. Springer. 4th International Workshop, CCA 2000, Swansea, UK, September 2000.

4. Robert D. Skeel. What makes molecular dynamics work? SIAM Journal on Scientific Computing, 31:1363-1378, 2009.

5. Christoph Spandl. Computational complexity of iterated maps on the interval. Mathematics and Computers in Simulation, 82:1459-1477, 2012.

6. Wilfred F. van Gunsteren and Alan E. Mark. Validation of molecular dynamics simulation. Journal of Chemical Physics, 108:6109-6116, 1998. 


\title{
Strong properness of dyadic subbases
}

\author{
Yasuyuki Tsukamoto* \\ Hideki Tsuiki ${ }^{\dagger}$ \\ Department of Human Coexistence, Kyoto University, Japan.
}

June 9, 2014

\begin{abstract}
A dyadic subbase $S$ of a topological space $X$ is a subbase which is composed of a countable collection of pairs of open subsets which are exteriors of each other. If a dyadic subbase $S$ is proper, then we can construct a dcpo $D_{S}$ where $X$ is embedded. However, whether the dcpo $D_{S}$ is consistently complete or not depends not only on $S$ itself but also on the ordering of $S$. We give a characterization of $S$ which induces the consistent completeness of $D_{S}$ regardless of its ordering.
\end{abstract}

\section{Proper dyadic subbases}

Throughout this section, $X=(X, \mathfrak{O})$ is a second-countable Hausdorff space. Let $\mathbb{N}$ be the set of non-negative integers.

Definition 1.1. A dyadic subbase of $X$ is a map $S: \mathbb{N} \times\{0,1\} \rightarrow \mathfrak{O}$ such that

$\{S(n, a) \mid n \in \mathbb{N}, a \in\{0,1\}\}$ is a subbase of $X$,

$S(n, 0)$ and $S(n, 1)$ are the exteriors of each other, for all $n \in \mathbb{N}$.

We set $\mathbb{T}:=\{0,1, \perp\}$ where $\perp$ is called the bottom character meaning undefinedness. Let $\mathbb{T}^{\mathbb{N}}$ denote the set of maps from $\mathbb{N}$ to $\mathbb{T}$. We call an element $\sigma \in \mathbb{T}^{\mathbb{N}}$ a bottomed sequence. For a bottomed sequence $\sigma \in \mathbb{T}^{\mathbb{N}}$, we set $\operatorname{dom}(\sigma):=\{n \in \mathbb{N} \mid \sigma(n) \neq \perp\}$. If $\operatorname{dom}(\sigma)$ is a finite set, then we call it a finite bottomed sequence. The set of finite bottomed sequences is denoted by $\mathbb{T}^{*}$.

Definition 1.2. A dyadic subbase $S$ of $X$ is called proper if it satisfies

$$
\bigcap_{k \in \operatorname{dom}(\sigma)} \operatorname{cl} S(k, \sigma(k))=\mathrm{cl} \bigcap_{k \in \operatorname{dom}(\sigma)} S(k, \sigma(k)) \text { for all } \sigma \in \mathbb{T}^{*},
$$

where $\operatorname{cl} A$ denotes the closure of $A \subseteq X$.

\footnotetext{
*tsukamoto@i.h.kyoto-u.ac.jp

†tsuiki@i.h.kyoto-u.ac.jp
} 
Let $S$ be a proper dyadic subbase of $X$. We consider a partial order $\perp \sqsubseteq$ $0, \perp \sqsubseteq 1$, and a $T_{0}$-topology $\{\emptyset,\{0\},\{1\},\{0,1\}, \mathbb{T}\}$ on $\mathbb{T}$. We equip $\mathbb{T}^{\mathbb{N}}$ with the product order and the product topology. We have a topological embedding $\varphi_{S}: X \rightarrow \mathbb{T}^{\mathbb{N}}$ defined as

$$
\varphi_{S}(x)(n):=\left\{\begin{array}{ll}
0 & (x \in S(n, 0)) \\
1 & (x \in S(n, 1)) \\
\perp & \text { (otherwise) }
\end{array} \text { for } x \in X, n \in \mathbb{N} .\right.
$$

We set

$$
\begin{aligned}
K_{S} & :=\left\{\left.\varphi_{S}(x)\right|_{n} \mid x \in X, n \in \mathbb{N}\right\}, \\
D_{S} & :=\left\{\sigma \in \mathbb{T}^{\mathbb{N}} \mid(\forall n \in \mathbb{N})\left(\left.\sigma\right|_{n} \in K_{S}\right)\right\},
\end{aligned}
$$

where $\left.\sigma\right|_{n}$ denotes the bottomed sequence with $\operatorname{dom}\left(\left.\sigma\right|_{n}\right) \subseteq\{k \in \mathbb{N} \mid k<n\}$ and $\left.\sigma\right|_{n}(k)=\sigma(k)$ for $k<n$. The set $D_{S}$ is an algebraic pointed depo which is the ideal completion of $K_{S}$. The dcpo $D_{S}$ is not a Scott domain in general, i.e., $D_{S}$ may not be consistently complete. Moreover, even if $D_{S}$ is a Scott domain, changing the order of some pairs in $S$ might cause $D_{S}$ not to be consistently complete.

Let $S(n, \partial)$ be the common boundary of $S(n, 0)$ and $S(n, 1)$. Similarly to $\mathbb{T}^{*},\{0,1, \partial, \perp\}^{*}$ denotes the set of maps $\sigma \in\{0,1, \partial, \perp\}^{\mathbb{N}} \operatorname{such}$ that $\operatorname{dom}(\sigma):=$ $\{n \in \mathbb{N} \mid \sigma(n) \neq \perp\}$ is finite.

Definition 1.3. We say that a dyadic subbase $S$ is strongly proper if it satisfies

$$
\mathrm{cl} \bigcap_{k \in \operatorname{dom}(\sigma)} S(k, \sigma(k))=\bigcap_{k \in \operatorname{dom}(\sigma)} \operatorname{cl} S(k, \sigma(k)) \text { for all } \sigma \in\{0,1, \partial, \perp\}^{*} .
$$

We show that $D_{S}$ is a Scott domain regardless of the ordering of $S$, if and only if $S$ is strongly proper.

Theorem 1.4. Let $S$ be a proper dyadic subbase of a Hausdorff space $X$. The following are equivalent.

1. $S$ is strongly proper.

2. For every permutation $\pi: \mathbb{N} \rightarrow \mathbb{N}, D_{S \pi}$ is a Scott domain, where $S \pi$ is another dyadic subbase given by $S \pi(n, a):=S(\pi(n), a)(n \in \mathbb{N}, a \in$ $\{0,1\})$.

\section{References}

[1] Tsuiki, H., Tsukamoto, Y., Domain representations induced by dyadic subbases, LMCS to apper, available at arXiv:1401.1393v1, 2014. 


\title{
Same for Bishop, different for Weihrauch
}

\author{
Kazuto Yoshimura \\ k.yoshimura@jaist.ac.jp \\ Japan Advanced Institute of Science and Technology
}

The Weihrauch lattice is a degree structure whose underlying reducibility requires uniform computability [2]. It had been investigated under purely computable analytic motivations; however, it has recently be claimed that there should be a close relationship between constructive reverse mathematics [6] and the classification of Weihrauch degrees.

Such relationship was originally suggested by G. Gherardi and A. Marcone in their paper [5]. They referred only to Friedman-Simpson's reverse mathematics, a different version of reverse mathematics from constructive reverse mathematics, however they provide fundamental observations on the connection to a logical approach. Afterwards V. Brattka and G. Gherardi started their synthetic project of classifying non-constructive principles in the Weihrauch lattice [2]; the project will be referred as BGM-program here. Nice analogies have been found between results of constructive reverse mathematics and those of BGM-program [3], [4].

This research aims to clarify the formal relationship between constructive reverse mathematics and BGM-program. To explain our thesis concisely suppose the following two fictional persons; one admits all derivations; the other one admits only those derivations which provide witnesses. Let us refer to the former one as Bishop and to the latter one as Weihrauch. Our thesis is that the formal relationship can be captured by the relationship between Bishop and Weihrauch.

Our main result is a sound interpretation from (a fragment of) a version of constructive theory, an extension of Heyting arithmetic, into the Weihrauch lattice. Using the interpretation, for example, we can find an alternative proof of the separation between LLPO ( $\Sigma_{1}^{0}$ De Morgan's law) and (W)LPO ((weak) $\Sigma_{1}^{0}$ law of excluded middle) over Heyting arithmetic which was originally shown in [1]. In particular, it is obtained as a consequence of a separation result in the Weihrauch lattice.

The main result is shown by a combination of two theories concerning the syntactic and semantic aspects, respectively. Let us explain how the two theories are related to our thesis.

As the results on syntactic aspects are concerned, we provide a version of witness extraction. A typical example of witness extraction is given by existence property of intuitionistic logic i.e. the property that we find a term $t$ which makes the formula $\psi[t / x]$ derivable with empty assumption whenever the formula $\exists x . \psi$ is derivable with empty assumption. On the other hand our witness extraction works, dually, when a formula is derivable assuming a formula of the form $\forall x . \psi$. In our case it is impossible to extract exactly one witness in general but a relaxed formulation can still be considered. We propose such a relaxed formulation of witness extraction and prove it using the Cut-Elimination Theorem [8]. 
Unfortunately our witness extraction needs several technical conditions on figures of sequents which are quite heavy in practice. To extend the scope of application, we consider transformations of sequents which do not change derivability, however which make our witness extraction succeed. In other words we try to define a transformation so that a given sequent and the resulting transformed sequent are the same for Bishop but different for Weihrauch, and furthermore, they agree on the derivability of the resulting transformed sequent. This strategy works certainly and it will be used for the main result.

As the results on semantic aspects are concerned, we introduce an operator which generates a degree structure, called an abstract Weihrauch degree structure, for a given fibration. Fibrations are fundamental structures from categorical logic which are frequently used to define semantics for various type theories [7]. If the given fibration is under a technical assumption, the generated abstract Weihrauch degree structure forms a bounded distributive lattice; in such a case we call it an abstract Weihrauch lattice. In particular, the Weihrauch lattice is embeddable into a suitable instance of abstract Weihrauch lattices.

There is a well-known semantics of SIL (Simply typed Intuitionistic Logic) given by first order fibration [7]. The semantics can be regarded as a sophisticated abstraction of Kleene's realizability interpretation. We utilize its soundness and abstract Weihrauch lattices for the main result. In particular they are used to show that witnesses of derivability corresponds to witnesses of Weihrauch reducibility. The syntactic theory and the semantic theory meet at this point.

\section{References}

1. Yohji Akama, Stefano Berardi, Susumu Hayashi, and Ulrich Kohlenbach. An arithmetical hierarchy of the law of excluded middle and related principles. In Harald Ganzinger, editor, Proc. of the 19th Annual IEEE Symposium on Logic in Computer Science (LICS 2004), pages 192-201. IEEE Computer Society Press, 2004.

2. Vasco Brattka and Guido Gherardi. Effective choice and boundedness principles in computable analysis. Bulletin of Symbolic Logic, 17(1):73-117, 2011.

3. Vasco Brattka and Guido Gherardi. Weihrauch degrees, omniscience principles and weak computability. J. Symb. Log., 76(1):143-176, 2011.

4. Vasco Brattka, Guido Gherardi, and Alberto Marcone. The bolzano-weierstrass theorem is the jump of weak könig's lemma. Ann. Pure Appl. Logic, 163(6):623$655,2012$.

5. Guido Gherardi and Alberto Marcone. How incomputable is the separable hahnbanach theorem? Electr. Notes Theor. Comput. Sci., 221:85-102, 2008.

6. Hajime Ishihara. Constructive reverse mathematics: compactness properties. In In Crosilla/Schuster: From sets and types to Topology and Analysis, pages 245-267. Oxford University Press, 2005.

7. Bart Jacobs. Categorical Logic and Type Theory. Number 141 in Studies in Logic and the Foundations of Mathematics. North Holland, Amsterdam, 1999.

8. Anne S. Troelstra and Helmut Schwichtenberg. Basic Proof Theory (2Nd Ed.). Cambridge University Press, New York, NY, USA, 2000. 



\section{Author Index}

van Ackerern, Alexander 20

Ackerman, Nathanael $\quad 13$

Bauer, Andrej 16

Brattka, Vasco 18, 51

Brauße, Franz 20

de Brecht, Matthew $\quad 1$

Briggs, Keith $\quad 22$

$\begin{array}{ll}\text { Collins, Pieter } & 25\end{array}$

Duracz, Jan $\quad 39,41$

Escardó, Martín $\quad 4$

Farjudian, Amin $\quad 41$

Franklin, Johanna $\quad 7$

Freer, Cameron $\quad 13$

$\begin{array}{ll}\text { Gherardi, Guido } & 18\end{array}$

Hendtlass, Matt $\quad 26$

Hölzl, Rupert $\quad 18$

Iljazović, Zvonko $\quad 29$

Kawamura, Akitoshi $\quad 31,34$

Kihara, Takayuki $\quad 36$

Konečný, Michal $\quad 39,41$

Korovina, Margarita $\quad 20$

Lubarsky, Robert $\quad 26$

McNicholl, Timothy

Melnikov, Alexander $\quad 9$

Ménissier-Morain, Valérie $\quad 42$

de Miguel Casado, Gregorio $\quad 45$

Müller, Norbert Th. 20

$\begin{array}{ll}\text { Ostrovsky, Alexey } & 47\end{array}$

Pape, Martin $\quad 49$

Pauly, Arno $\quad 11,52$

Pažek, Bojan $\quad 29$ 
Rakotoniaina, Tahina

Le Roux, Stéphane

52

Roy, Daniel

Schröder, Matthias

Spandl, Christoph

Steinberg, Florian

Taha, Walid

41

Thies, Holger

31

Tsuiki, Hideki

58

Tsukamoto, Yasuyuki

58

$\mathrm{Xu}$, Chuangjie

4

Yamamoto, Kentarô

34

Yoshimura, Kazuto

16,60 